\title{
Exact $\mathrm{WKB}$ and abelianization for the $T_{3}$ equation
}

\author{
Lotte Hollands ${ }^{1}$ and Andrew Neitzke ${ }^{2}$ \\ ${ }^{1}$ Department of Mathematics, Heriot-Watt University \\ ${ }^{2}$ Department of Mathematics, University of Texas at Austin
}

\begin{abstract}
We describe the exact WKB method from the point of view of abelianization, both for Schrödinger operators and for their higher-order analogues (opers). The main new example which we consider is the " $T_{3}$ equation," an order 3 equation on the thrice-punctured sphere, with regular singularities at the punctures. In this case the exact WKB analysis leads to consideration of a new sort of Darboux coordinate system on a moduli space of flat SL(3)-connections. We give the simplest example of such a coordinate system, and verify numerically that in these coordinates the monodromy of the $T_{3}$ equation has the expected asymptotic properties. We also briefly revisit the Schrödinger equation with cubic potential and the Mathieu equation from the point of view of abelianization.
\end{abstract}

\section{Contents}

1 Introduction 3

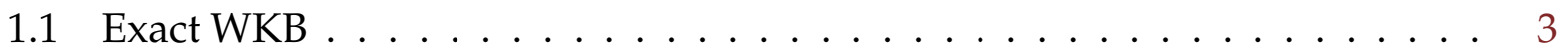

1.2 Abelianization . . . . . . . . . . . . . . . . 3

1.3 Voros symbols for Schrödinger equations . . . . . . . . . . . . . . 4

1.4 Exact WKB for order 3 equations . . . . . . . . . . . . . 5

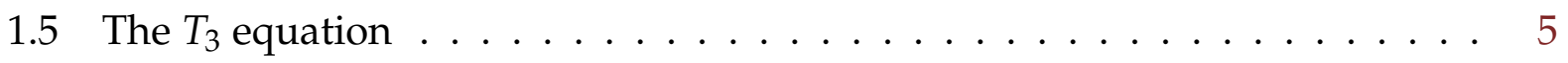

1.6 Integral equations and analytic structures $\ldots \ldots \ldots \ldots$

1.7 Supersymmetric QFT . . . . . . . . . . . . . . . . . . . . . . . . . . . . . . . . . .

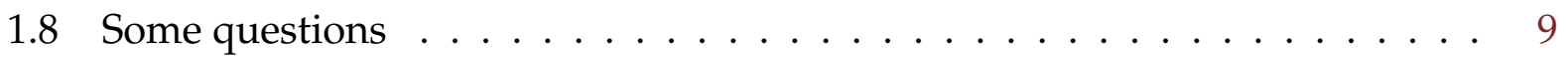

2 Exact WKB for Schrödinger equations 11

2.1 WKB solutions . . . . . . . . . . . . . . . . . . . . . 12

2.2 Abelianization . . . . . . . . . . . . . . . . 14

2.3 Gluing across the Stokes graph . . . . . . . . . . . . . . . . 14 


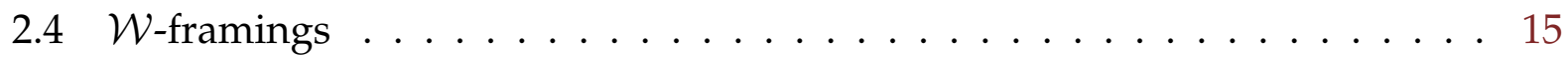

2.5 Spectral coordinates and their properties . . . . . . . . . . . 17

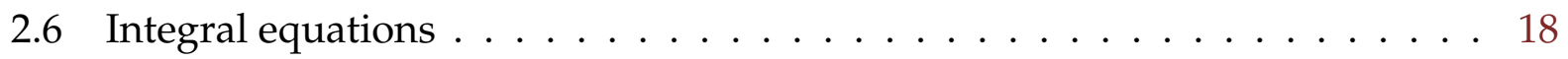

3 Exact WKB for Schrödinger operators with cubic potential 20

3.1 A Stokes graph . . . . . . . . . . . . . . . . 21

3.2 The spectral coordinates . . . . . . . . . . . . . . . 21

3.3 Analytic continuation . . . . . . . . . . . . . . . . 22

3.4 The regular pentagon . . . . . . . . . . . . . . . . 23

3.5 Integral equations for spectral coordinates . . . . . . . . . . . . . 24

4 Exact WKB for the Mathieu equation 25

4.1 Exponential coordinate . . . . . . . . . . . . . . . . . . 25

4.2 A simple Stokes graph . . . . . . . . . . . . . . . . . . . . . 25

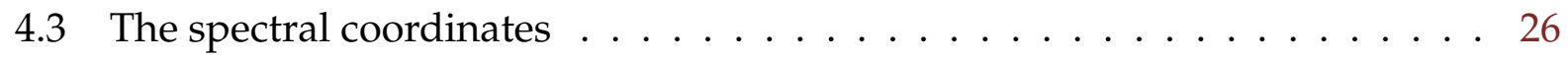

4.4 Application: bound states $\ldots \ldots \ldots \ldots \ldots$

4.5 Analytic continuation . . . . . . . . . . . . . . . . . . 27

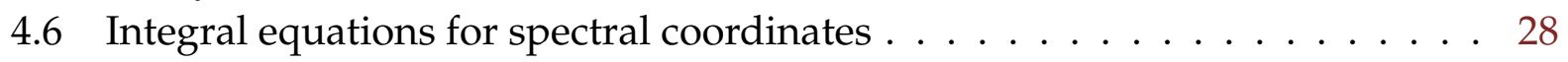

4.7 Another Stokes graph . . . . . . . . . . . . . . . . . . . . . . . 31

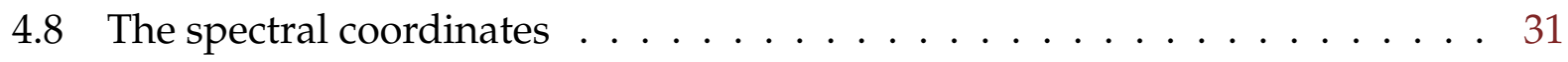

4.9 Application: quasiperiodic solutions . . . . . . . . . . . . 32

5 Exact WKB for higher order equations 34

5.1 WKB solutions . . . . . . . . . . . . . . . . . . . . 34

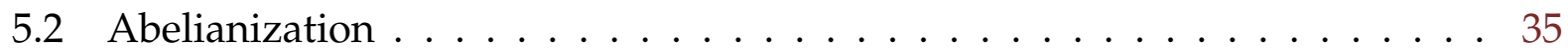

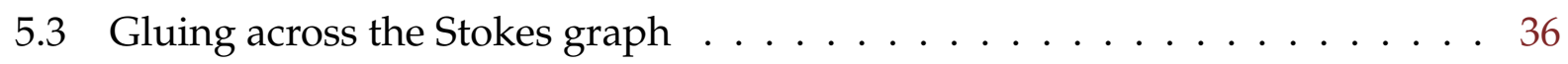

5.4 Spectral coordinates . . . . . . . . . . . . . . . . 36

6 Exact WKB for the $T_{3}$ equation 36

6.1 A simple Stokes graph . . . . . . . . . . . . . . . . . . 37

6.2 The abelianization problem for the $T_{3}$ equation $\ldots \ldots \ldots 38$

6.3 Projective bases in special position . . . . . . . . . . . . . . . 39

6.4 The spectral coordinates . . . . . . . . . . . . . . . . . 42

6.5 Spectral coordinates for the continuous family of abelianizations . . . . . . 43

6.6 The monodromy matrices . . . . . . . . . . . . . . . . 43

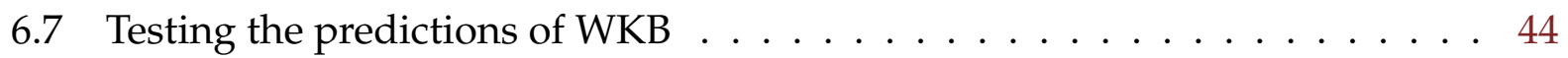

6.8 Analytic continuation . . . . . . . . . . . . . . . . . 46

6.9 The uniformization point . . . . . . . . . . . . . . . . . 48

6.10 Integral equations . . . . . . . . . . . . . . . . . . . . 49

6.11 Spectral problem . . . . . . . . . . . . . . . . . 53 
$\begin{array}{lll}7 & \text { Supersymmetric field theory } & 54\end{array}$

7.1 Opers and QFT of class $S \ldots \ldots \ldots \ldots \ldots$

7.2 Spectral coordinates as vevs . . . . . . . . . . . . . . 55

7.3 Scaling line defects . . . . . . . . . . . . . . . . . . 55

7.4 Opers and instanton counting . . . . . . . . . . . . . . . 57

A Computations of spectral coordinates 58

A.1 Computations for the cubic potential . . . . . . . . . . . . 58

A.2 Computations for the Mathieu equation . . . . . . . . . . . . . . . . 59

A.3 Computations for the $T_{3}$ equation . . . . . . . . . . . . . . . . 61

\section{Introduction}

\subsection{Exact WKB}

The exact WKB method is a scheme for studying the monodromy (or bound states, or more generally Stokes data) of linear scalar differential equations. This method was initiated in $[1,2,3,4]$ and subsequently developed in a large body of literature. Its origin is in the study of Schrödinger equations, of the form

$$
\left[\hbar^{2} \partial_{z}^{2}+P(z)\right] \psi(z)=0,
$$

where $P(z)$ is holomorphic or meromorphic; most of the literature is concerned with this case. For some useful reviews see [5, 6, 7]. More recently the exact WKB investigation of higher-order analogues of Schrödinger equations has been taken up, e.g. in $[8,9,10,11,12$, $13,14]$.

\subsection{Abelianization}

The method of $[15,16]$ leads to a new geometric reformulation of exact WKB, both for Schrödinger operators and their higher-order analogues. In this reformulation, the key step in exact WKB is a process of "abelianization" which replaces a flat $\mathrm{SL}(K)$-connection $\nabla$ over a surface $C$ by a flat GL(1)-connection $\nabla^{\mathrm{ab}}$ over a $K$-fold covering $\Sigma \rightarrow C$. ${ }^{1}$ Some aspects of this abelianization process and its relation to exact WKB have been further developed in $[17,18,19,20]$.

In Section 2 we review the exact WKB method for Schrödinger operators, i.e. the $K=2$ case, from the perspective of abelianization. The aim is not to break any really new ground, but just to explain the theory from the abelianization point of view, which is a bit different from the conventional language of exact WKB.

\footnotetext{
${ }^{1}$ Throughout this paper $\operatorname{SL}(K)$ means $\operatorname{SL}(K, \mathbb{C})$, and $\operatorname{GL}(1)$ means $\operatorname{GL}(1, \mathbb{C})=\mathbb{C}^{\times}$.
} 


\subsection{Voros symbols for Schrödinger equations}

The exact WKB analysis of Schrödinger equations revolves around certain complexvalued functions $\mathcal{X}_{\gamma}(\hbar)$ known as the Voros symbols. ${ }^{2}$ In the language of abelianization, the $\mathcal{X}_{\gamma}(\hbar)$ are the holonomies of the GL(1)-connection $\nabla^{\mathrm{ab}}$ around 1-cycles $\gamma$ on the double cover $\Sigma$.

The $\mathcal{X}_{\gamma}(\hbar)$ can be expressed as products of Wronskians of distinguished local solutions $\psi_{i}(z, \hbar)$ of (1.1). The solutions $\psi_{i}(z, \hbar)$ have a dual role:

- On the one hand, the $\psi_{i}(z, \hbar)$ are produced by Borel resummation of the perturbative WKB series. As a result, one has good control over their behavior as $\hbar \rightarrow 0$, which gives good control over the behavior of $\mathcal{X}_{\gamma}(\hbar)$ as $\hbar \rightarrow 0$.

- On the other hand, the $\psi_{i}(z, \hbar)$ can be characterized intrinsically: either as asymptotically decaying solutions as $z$ approaches a singularity, or as eigenvectors of the monodromy as $z$ goes around a loop. This allows one to identify the $\mathcal{X}_{\gamma}(\hbar)$ as familiar coordinate functions on a moduli space of flat SL(2)-connections. In a generic enough situation these are the "Fock-Goncharov coordinates" introduced in [21], as explained in $[15,7,17,22]$. In less generic situations, as discussed in [15, 17], one can get the "exponentiated complexified Fenchel-Nielsen coordinates" studied in [23, 24, 25], or other slight variants.

The combination of these two points of view on the $\mathcal{X}_{\gamma}(\hbar)$ is responsible for much of the power of the exact WKB method.

In this paper we revisit this story in two examples, again with the aim of showing how exact WKB works in the language of abelianization, and paving the way for the higher-order case:

- First, in Section 3, we discuss the Schrödinger equation with cubic potential. This is an instance of (1.1) with $P(z)=z^{3}-u$. We treat this example relatively briefly. We consider only the choice $u=1$ and real $\hbar$, for which the $\mathcal{X}_{\gamma}(\hbar)$ are Fock-Goncharov coordinates.

- Second, in Section 4, we discuss the Mathieu equation. This is an instance of (1.1) with $P(z)=\frac{1}{z^{3}}-\frac{2 E-\frac{1}{4} \hbar^{2}}{z^{2}}+\frac{1}{z}$. We focus on the cases of real $\hbar>0$ and $E>1$ or $E<-1$. For $E<-1$ the $\mathcal{X}_{\gamma}(\hbar)$ turn out to be Fenchel-Nielsen coordinates, and we explain their application to the bound state problem for the modified Mathieu equation; for $E>1$ the $\mathcal{X}_{\gamma}(\hbar)$ turn out to be a slight variant of the Fock-Goncharov coordinates, and we explain their application to the quasiperiodic solutions of the ordinary Mathieu equation. In either case we do not do anything really new, except perhaps that we give a new version of the exact quantization condition for the Mathieu equation, (4.25), and use it to derive the width of the gaps at small $\hbar$.

\footnotetext{
${ }^{2}$ In the main text we will distinguish several different variants of the functions $\mathcal{X}_{\gamma}$. The functions $\mathcal{X}_{\gamma}^{\text {intro }}$ we use in the introduction are related to those appearing in the main text by $\mathcal{X}_{\gamma}^{\text {intro }}(\hbar)=\mathcal{X}_{\gamma}^{\vartheta=\arg \hbar}(\hbar)$.
} 


\subsection{Exact WKB for order 3 equations}

The next natural test bed is the case of SL(3)-opers: this means order 3 equations of the general form

$$
\left[\partial_{z}^{3}+\hbar^{-2} P_{2} \partial_{z}+\left(\hbar^{-3} P_{3}+\frac{1}{2} \hbar^{-2} P_{2}^{\prime}\right)\right] \psi(z)=0 .
$$

In Section 5 we describe an extension of the exact WKB method to this case, again in the language of abelianization. This extension comes from combining the methods of [16] with the scaling limit of [26], now applied to families of SL(3)-connections.

As in the order 2 case, the theory is founded on the existence of distinguished local solutions $\psi_{i}(z, \hbar)$ of (1.2), with $\hbar \rightarrow 0$ asymptotics given by the WKB series. In contrast to the order 2 case, however, as far as we know, there are not yet theorems guaranteeing the existence of these local solutions. Thus the higher-order exact WKB method is not yet on solid footing.

Nevertheless, we press on, making the assumption that the $\psi_{i}(z, \hbar)$ do exist. Then, as before, one can use them to construct functions $\mathcal{X}_{\gamma}(\hbar)$, which we call spectral coordinates because of their relation to abelianization; one might also have called them higher-order Voros symbols. Now the question arises: can we identify the $\mathcal{X}_{\gamma}(\hbar)$ as some concrete coordinate functions on moduli of flat SL(3)-connections - or, essentially equivalently, can we give an intrinsic characterization of the $\psi_{i}(z, \hbar)$ in terms of their monodromy properties?

For some examples of equations (1.2), the expected picture is well understood, and similar to the order 2 case. One such situation arises if $C$ is a punctured surface, $P_{2}$ is meromorphic and generic with generic residues, and $P_{3}$ is small compared to $P_{2}$. In this case the $\psi_{i}(z, \hbar)$ in most of $C$ can be described by beginning with the filtrations induced by the asymptotic growth rate at the punctures, and then using the linear algebra of "snakes" as introduced by Fock-Goncharov [21, 27]. The $\mathcal{X}_{\gamma}(\hbar)$ then turn out to be higher-rank FockGoncharov coordinates. Another such situation arises if $\left(P_{2}, P_{3}\right)$ is a generalized Strebel pair of length-twist type as defined in [18]; then the $\psi_{i}(z, \hbar)$ can be characterized as monodromy eigenvectors, and the $\mathcal{X}_{\gamma}(\hbar)$ turn out to be "higher length-twist" coordinates generalizing Fenchel-Nielsen. In this paper we do not revisit these cases.

\subsection{The $T_{3}$ equation}

Instead, in Section 6, we turn our attention to the $T_{3}$ equation. This is a specific instance of (1.2), defined on the Riemann surface $C=\mathbb{C P}^{1}$ with three generic regular singularities ("full punctures" in the physics literature), and depending on a parameter $u \in \mathbb{C}$ : namely, we take in (1.2)

$$
P_{2}=\frac{9 \hbar^{2} z}{\left(z^{3}-1\right)^{2}}, \quad P_{3}=\frac{u}{\left(z^{3}-1\right)^{2}} .
$$


This equation is a particularly interesting test case. One way to understand this is to remark that this family of opers corresponds (in the sense of Subsection 1.7 below) to a specific $\mathcal{N}=2$ superconformal quantum field theory, the Minahan-Nemeschansky theory with flavor symmetry $E_{6}[28,29]$, which is known to be difficult to study by conventional Lagrangian field theory methods.

As expected, in this case we meet new difficulties. One source of these difficulties is that the Stokes graphs can be rather wild for general $(u, \hbar)$. We thus restrict ourselves to only the simplest situation, which arises when $u^{\prime}=u / \hbar^{3}$ is real and positive; in this case the Stokes graph is actually very simple. It is shown in Figure 10 below.

Then we find that the $\psi_{i}(z, \hbar)$ are solutions of an interesting linear algebra problem: relative to the local basis $\left\{\psi_{1}, \psi_{2}, \psi_{3}\right\}$ near $z=0$, the monodromies $\mathbf{A}, \mathbf{B}, \mathbf{C}$ around the three punctures must have zeroes in specific positions,

$$
\begin{aligned}
\mathbf{A} & =\left(\begin{array}{lll}
* & 0 & * \\
* & * & * \\
* & * & *
\end{array}\right), \quad \mathbf{B}=\left(\begin{array}{lll}
* & * & * \\
* & * & 0 \\
* & * & *
\end{array}\right), \quad \mathbf{C}=\left(\begin{array}{lll}
* & * & * \\
* & * & * \\
0 & * & *
\end{array}\right), \\
\mathbf{A}^{-1} & =\left(\begin{array}{lll}
* & * & * \\
0 & * & * \\
* & * & *
\end{array}\right),
\end{aligned}
$$

The best approach we have found to this linear algebra problem involves a bit of algebraic geometry, as we describe in Subsection 6.3: we reduce the problem to finding fixed points of a certain degree 64 birational automorphism of $\mathbb{C P}^{2}$, and then identify these fixed points with singularities in the fibers of a certain rational elliptic surface.

At any rate, once this problem has been solved, we can then compute the spectral coordinates $\mathcal{X}_{\gamma}(\hbar)$ for the $T_{3}$ equation. The concrete formulas are given in (6.18) below, reproduced here: for a basis $\left\{\gamma_{A}, \gamma_{B}\right\}$ of $H_{1}(\Sigma, \mathbb{Z}),{ }^{3}$

$$
\begin{aligned}
\mathcal{X}_{\gamma_{A}} & =\frac{\left[\psi_{2}, \psi_{3}, \psi_{1}\right]}{\left[\mathbf{C}^{-1} \psi_{3}, \mathbf{A} \psi_{2}, \psi_{1}\right]} \\
\mathcal{X}_{\gamma_{B}} & =\sqrt{-\frac{\left[\mathbf{C} \psi_{1}, \mathbf{B}^{-1} \psi_{2}, \psi_{3}\right]\left[\mathbf{C} \psi_{1}, \psi_{1}, \psi_{3}\right]\left[\psi_{2}, \mathbf{A} \psi_{2}, \psi_{1}\right]\left[\mathbf{B} \psi_{3}, \mathbf{A}^{-1} \psi_{1}, \psi_{2}\right]\left[\mathbf{B} \psi_{3}, \psi_{3}, \psi_{2}\right]}{\left[\psi_{2}, \mathbf{B}^{-1} \psi_{2}, \psi_{3}\right]\left[\mathbf{C}^{-1} \psi_{3}, \mathbf{A} \psi_{2}, \psi_{1}\right]\left[\mathbf{C}^{-1} \psi_{3}, \psi_{3}, \psi_{1}\right]\left[\psi_{1}, \psi_{3}, \psi_{2}\right]\left[\psi_{1}, \mathbf{A}^{-1} \psi_{1}, \psi_{2}\right]}}
\end{aligned}
$$

where $\left[\psi, \psi^{\prime}, \psi^{\prime \prime}\right]$ means the Wronskian of the three solutions $\psi, \psi^{\prime}, \psi^{\prime \prime}$. This gives a local Darboux coordinate system on the moduli space of flat SL(3)-connections with unipotent holonomy on the thrice-punctured sphere. As far as we know, this coordinate system has not been considered before. What our computations say is that these particular coordinates arise naturally from the WKB analysis of the equation (1.2), (1.3).

\footnotetext{
${ }^{3}$ Here and elsewhere in this paper, unless explicitly noted, $\sqrt{\cdot}$. denotes the principal branch of the square root.
} 
Combining our conjectures and computations, one can extract a concrete prediction: the quantities (1.5), computed from the monodromy of the $T_{3}$ equation, should have a specific asymptotic series expansion, with leading behavior $\mathcal{X}_{\gamma} \sim \exp \left(Z_{\gamma} / \hbar\right)$, as $\hbar \rightarrow 0$ in an appropriate sector. Here the constants $Z_{\gamma} \in \mathbb{C}$ are periods, $Z_{\gamma}=\oint_{\gamma} P_{3}^{1 / 3}$, given explicitly in (6.24) below. We have implemented this computation numerically and find very good agreement (see e.g. Figure 14 below). We regard this as evidence that the higher-order exact WKB method indeed works.

\subsection{Integral equations and analytic structures}

A relatively recent development in the exact WKB method is the discovery that the functions $\mathcal{X}_{\gamma}(\hbar)$ are, quite generally, solutions of integral equations in the $\hbar$-plane. A general form of these integral equations was formulated in [26] (see (2.19) below), generalizing some cases which had been known before. In particular, the equations closely resemble the thermodynamic Bethe ansatz, and some cases literally match with the high-temperature (chiral) limit of the thermodynamic Bethe ansatz for specific integrable models; these cases had been studied as part of the ODE-IM correspondence, explained in e.g. [30, 31].

One way to motivate these equations is to argue that their solutions solve a certain Riemann-Hilbert problem: they have the same analytic structure and $\hbar \rightarrow 0$ asymptotic properties as the desired functions $\mathcal{X}_{\gamma}(\hbar)$ have. One hopes that these properties are sufficient to characterize $\mathcal{X}_{\gamma}(\hbar) .{ }^{4}$

The general idea of determining the $\mathcal{X}_{\gamma}(\hbar)$ from their analytic properties has appeared before in the exact WKB literature, e.g. in [3] under the name "analytic bootstrap." In another direction, the same Riemann-Hilbert problem has been studied recently in relation to the topological string [32].

In various sections of this paper we consider integral equations for our $\mathcal{X}_{\gamma}(\hbar)$ :

- In Subsection 3.5 we review the integral equations obeyed by $\mathcal{X}_{\gamma}(\hbar)$ for the Schrödinger equation with cubic potential. In this case the $\mathcal{X}_{\gamma}(\hbar)$ are Fock-Goncharov coordinates. This case is by now reasonably well understood in the literature; it was discussed already in [30], in [26], and more recently in [33].

- In Subsection 4.6 we propose integral equations for $\mathcal{X}_{\gamma}(\hbar)$ for the Mathieu equation, in the case where $\mathcal{X}_{\gamma}(\hbar)$ are complexified exponentiated Fenchel-Nielsen coordinates. This case is somewhat more difficult; we find definite equations, which do seem to be satisfied by the $\mathcal{X}_{\gamma}(\hbar)$ in numerical experiments, but we are not able to use the equations to compute the $\mathcal{X}_{\gamma}(\hbar)$ directly.

- Finally in Subsection 6.10 we write one version of the integral equations for the $\mathcal{X}_{\gamma}(\hbar)$ of the $T_{3}$ equation. Here, in order to determine the equations completely, one needs to

\footnotetext{
${ }^{4}$ For a more elementary example, if a function $x(\hbar)$ is known to be holomorphic for $\hbar \in \mathbb{C}^{\times}, x(\hbar) \rightarrow c$ as $\hbar \rightarrow 0$, and $x(\hbar)$ is bounded as $\hbar \rightarrow \infty$, then we can conclude $x(\hbar)=c$.
} 
find a closed formula for a certain transformation $\mathbf{S}_{0, \frac{\pi}{3}}$ relating two different branches of $\mathcal{X}_{\gamma}(\hbar)$; we formulate this problem carefully but do not solve it. We also explain how one can approximate $\mathbf{S}_{0, \frac{\pi}{3}}$ using some integer invariants previously computed in [34] (BPS indices in the Minahan-Nemeschansky $E_{6}$ theory), and give some numerical evidence that this approximation works.

All of these analyses just barely scratch the surface; there is much more to do here.

A closely related issue is that of the analytic structure of the maximal analytic continuation of $\mathcal{X}_{\gamma}(\hbar)$ from a given initial $\hbar$. Zeroes, poles, and branch cuts can all occur:

- In Subsection 3.3 we briefly recall the analytic properties of the Fock-Goncharov coordinates $\mathcal{X}_{\gamma}(\hbar)$ for the Schrödinger equation with cubic potential. These are relatively simple: the maximal analytic continuation is defined on a fivefold cover branched only at $\hbar=0$, with a concrete monodromy action (3.4). The $\mathcal{X}_{\gamma}(\hbar)$ can also have poles or zeroes, which come from bound states of the Schrödinger equation; they occur in infinite discrete families.

- In Subsection 4.5 we describe the analytic properties of the complexified exponentiated Fenchel-Nielsen coordinates $\mathcal{X}_{\gamma}(\hbar)$ for the Mathieu equation in the regime $E<-1$. These are a bit more complicated: there is infinite-order monodromy (4.11) around $\hbar=0$, and also order-2 monodromy (4.12) around an infinite discrete family of other points. The latter points are analytically continued versions of the edges of the bands/gaps in the Mathieu spectrum. In this case we did not explore the positions of poles or zeroes.

- In Subsection 6.8 we consider the analytic properties of our new coordinates $\mathcal{X}_{\gamma}(\hbar)$ for the $T_{3}$ equation. The picture we find, through numerical experimentation, is that the maximal analytic continuation lives on a threefold cover, with order- 3 monodromy around $\hbar=0$, and order- 2 monodromy around 6 other points. In terms of the coordinate $u^{\prime}=u / \hbar^{3}$, the picture is simpler: there is only order- 2 branching, around the points $u^{\prime}= \pm u_{*}^{\prime}$ where $u_{*}^{\prime} \approx 0.041992794$. The $\mathcal{X}_{\gamma}(\hbar)$ can also have poles or zeroes, which numerically do appear to occur, in infinite discrete families.

\subsection{Supersymmetric QFT}

Over the last decade it has turned out that exact WKB is closely connected to $\mathcal{N}=2$ supersymmetric quantum field theory in four dimensions. This work was motivated by an attempt to understand these connections better. They arose in two different ways:

- On the one hand, [35] discovered a new connection between Nekrasov's $\Omega$-background partition function $\mathbf{Z}$ in $\mathcal{N}=2$ theories and quantum integrable systems. For $\mathcal{N}=2$ theories of class $S$, the AGT correspondence says $\mathbf{Z}$ is related to Liouville conformal blocks on a Riemann surface $C$ [36], while the quantum integrable systems turned 
out to be spectral problems for Schrödinger equations (1.1) on $C$. The investigation of this connection between Schrödinger equations, Liouville conformal blocks and topological strings was carried out using WKB methods beginning in [37, 38]. This connection has led to a flow of ideas in both directions. For example, it has been proposed that using exact WKB one can obtain "nonperturbative" information about $\mathbf{Z}$, e.g. [39, 40,41]; also, techniques from the study of $\mathbf{Z}$, such as the holomorphic anomaly equations, have been imported back to WKB, e.g. [42, 43].

- On the other hand, studying BPS states and supersymmetric defects in $\mathcal{N}=2$ theories of class $S,[15,16]$ were led to develop a version of exact WKB which applies to a slightly different sort of equation: instead of the 1-parameter families (1.1) or (1.2) parameterized by $\hbar \in \mathbb{C}^{\times},[15,16]$ treat a 2-parameter family of covariant constancy equations for flat $\mathrm{SL}(K)$-connections $\nabla_{R, \zeta}$, parameterized by $R \in \mathbb{R}_{+}$and $\zeta \in \mathbb{C}^{\times}:{ }^{5}$

$$
\nabla_{R, \zeta} \psi(z)=0 .
$$

Despite the difference between (1.1) and (1.6), the geometric structures which appear in their exact WKB analysis are the same; in particular the Stokes graphs in exact WKB are the same as the spectral networks in [16]. A reason for this was conjectured in [26], as follows: in the case $K=2$, taking the scaling limit $R \rightarrow 0, \zeta \rightarrow 0$ while holding $\hbar=\zeta / R$ fixed reduces the 2-parameter family of equations (1.6) to the 1parameter family (1.1). For general $K \geq 2$, this scaling limit similarly reduces (1.6) to a 1-parameter family of SL $(K)$-opers, i.e. order $K$ linear scalar ODEs. This conjecture was proven in some cases in [44].

In this paper we mostly focus on questions internal to exact $\mathrm{WKB}$, using these developments in physics only as motivation. However, in the final section, Section 7, we return briefly to the question of what our computations mean for $\mathcal{N}=2$ field theory. We propose that the construction of the functions $\mathcal{X}_{\gamma}$ provided by exact $\mathrm{WKB}$ is related to a construction of supersymmetric local operators in the field theory in $\Omega$-background, and comment on the expected relation of the $\mathcal{X}_{\gamma}$ to the Nekrasov $\Omega$-background partition function $\mathbf{Z}$, motivated by the ideas of [25].

\subsection{Some questions}

This project has raised, at least in our minds, many unanswered questions. Here are some:

- In our study of the $T_{3}$ equation we consider only a specific Stokes graph, the simplest of infinitely many which occur at different points in the $u^{\prime}$-plane. Even for this Stokes

\footnotetext{
${ }^{5}$ The family of flat connections $\nabla_{R, \zeta}$ arises from a solution $(D, \varphi)$ of Hitchin's equations, through the formula $\nabla_{R, \zeta}=R \zeta^{-1} \varphi+D+R \zeta \varphi^{\dagger}$.
} 
graph the monodromy properties of the local WKB solutions turn out to involve a complicated linear algebra problem, which seems to require real work to solve (in Subsection 6.3). What kind of problem will appear at other points of the $u^{\prime}$-plane? Is there some systematic way of solving all of them at once?

- Similarly, what happens in other Minahan-Nemeschansky theories, like the $E_{7}$ or $E_{8}$ theories? Is there a uniform way of describing the $\mathcal{X}_{\gamma}$ and their behavior, or do we have to treat each example separately?

- In this paper we reformulate various aspects of exact WKB in the language of abelianization. One notable exception is the "P/NP relation" discussed recently in the WKB literature, e.g. [45, 46, 47, 48, 49, 42, 50,33]. Does this part of the story have a useful geometric reformulation in the language of abelianization?

- In our discussions of TBA-type integral equations in Subsection 4.6 and Subsection 6.10 we make some progress, but do not attain the ultimate goal, which would be to completely determine the monodromy of the oper in terms of these integral equations. It would be very interesting to push this project further. For the $T_{3}$ equation the main obstruction to doing so is that we have not understood the coordinate transformation $\mathbf{S}_{0, \frac{\pi}{3}}$ appearing in Subsection 6.10. Finding a closed form for this transformation would be very interesting in its own right since it would be equivalent to completely determining the BPS spectrum of the $E_{6}$ Minahan-Nemeschansky theory.

- In Subsection 6.8 we uncover an unexpectedly interesting analytic structure for the functions $\mathcal{X}_{\gamma}$ in the case of the $T_{3}$ equation. It is natural to ask what is the physical meaning in the $E_{6}$ Minahan-Nemeschansky theory of the nonperturbative monodromy we find around the points $u^{\prime}= \pm u_{*}^{\prime}$. (A similar question for pure $\mathcal{N}=2$ supersymmetric $U(N)$ gauge theory was discussed in [51], where the relevant physics was proposed to be the appearance of new massless fields in the theory in $\Omega$-background; perhaps the monodromy we have found has a similar meaning.) It would also be interesting to prove rigorously that there is no monodromy around any other points in the $u^{\prime}$-plane.

- In this paper we treat only the case of the $T_{3}$ equation with unipotent monodromy, corresponding to the massless Minahan-Nemeschansky theory. There is a natural perturbation to consider, taking semisimple monodromy instead of unipotent, corresponding to the mass-perturbed Minahan-Nemeschansky theory. It would be interesting to study this case systematically - in particular, to see how the analytic structure of the $\mathcal{X}_{\gamma}$ is modified in this case. (On general grounds we should expect that the structure could be more complicated; in the massless case the monodromy came ultimately from the fact that there were 4 discrete abelianizations of the $T_{3}$ equation; in the massive case there are 12 discrete abelianizations rather than 4.)

- The exact WKB analysis we describe in this paper for equations of order $K>2$ is still conjectural, mainly because it has not yet been proven that the local WKB series are 
Borel summable. It would be very interesting to close this gap, perhaps by extending the approach of Koike-Schäfke from the $K=2$ case, or by using the integral equations of [52].

- The Darboux coordinates we encounter on moduli of SL(3)-connections over the thrice-punctured sphere are new as far as we know. It would be interesting to understand explicitly their relation to other known Darboux coordinate systems on the same space, e.g. the Fock-Goncharov coordinates [21], the coordinates introduced by Goldman [53], or the coordinates obtained from conformal field theory in [54].

- Finally, as we discuss in Section 7, the exact WKB computations we make here should have a precise meaning in terms of $\mathcal{N}=2$ supersymmetric quantum field theories in the $\Omega$-background. We make some proposals in this direction, but to put these proposals on a firm footing would seem to require new constructions of supersymmetric local operators and boundary conditions compatible with the $\Omega$-background. It would be very interesting to develop this story further.

\section{Acknowledgements}

We thank Dylan Allegretti, Tom Bridgeland, Gerald Dunne, Dan Freed, Marco Gualtieri, Kohei Iwaki, Saebyeok Jeong, Anton Leykin, Marcos Mariño, Nikita Nikolaev, Shinji Sasaki, Bernd Sturmfels and Joerg Teschner for useful and enlightening discussions. LH's work is supported by a Royal Society Dorothy Hodgkin Fellowship. AN's work on this paper was supported by NSF grant DMS-1711692 and by a Simons Fellowship in Mathematics.

\section{Exact WKB for Schrödinger equations}

We consider a holomorphic Schrödinger equation, of the local form ${ }^{6}$

$$
\left[\hbar^{2} \partial_{z}^{2}+P(z, \hbar)\right] \psi(z)=0
$$

The equation (2.1) can be given a global meaning on a Riemann surface $C$ equipped with a spin structure and complex projective structure. In that case $\psi(z)$ must be interpreted as a section of $K_{C}^{-\frac{1}{2}}$, and $P(z, \hbar)$ as a meromorphic quadratic differential. All of our considerations extend to this situation. Nevertheless, most of the important constructions can be understood concretely in a single coordinate patch, and we will write them that way throughout.

\footnotetext{
${ }^{6}$ In comparing to the ordinary Schrödinger equation on the real line we would have $P=2(E-V)$.
} 


\subsection{WKB solutions}

The WKB method is often described in terms of distinguished local WKB solutions. In this section we briefly recall the construction of these solutions. (To forestall confusion we emphasize that the WKB solutions are exact solutions, not approximate solutions.)

Suppose we fix a contractible open set $U \subset C$, a local coordinate $z$ on $U$, and a point $z_{0} \in U$. A WKB solution of (2.1) on $U$ means a solution of the form

$$
\psi(z)=\exp \left(\hbar^{-1} \int_{z_{0}}^{z} \lambda(z) \mathrm{d} z\right) .
$$

For $\psi(z)$ to be a solution of (2.1), $\lambda$ must obey the Riccati equation,

$$
\lambda^{2}+P+\hbar \partial_{z} \lambda=0 .
$$

The first step in constructing such a $\lambda$ is to build a formal series solution $\lambda^{\text {formal }}$ of (2.3) in powers of $\hbar$. The order $\hbar^{0}$ part of (2.3) is

$$
y^{2}+p=0,
$$

where $y$ (resp. $p$ ) is the $\hbar^{0}$ term in $\lambda$ (resp. P). ${ }^{7}$ Thus we have a two-fold ambiguity, resolved by choosing one of the two square roots of $-p$. It will be important to keep careful track of this choice of square root. Thus we introduce the Riemann surface of $\sqrt{-p}$,

$$
\Sigma=\left\{y^{2}+p=0\right\} .
$$

$\Sigma$ is a branched double cover of $C$. A sheet of the covering $\Sigma$ corresponds to a choice of $y$ obeying (2.4). We use the generic labels $i, j$ to represent the sheets, and $y_{i}, y_{j}$ for the corresponding square roots of $-p .^{8}$

We now choose a sheet $i$, and consider a formal series solution $\lambda_{i}^{\text {formal }}$ of (2.3), where we choose the $\hbar^{0}$ term to be $y_{i}$. The higher-order expansion of $\lambda_{i}^{\text {formal }}$ is then uniquely fixed by (2.3), taking the form

$$
\lambda_{i}^{\text {formal }}=y_{i}+\sum_{n=1}^{\infty} \hbar^{n} \lambda_{i}^{\text {formal }, n} .
$$

For example, if $P$ is $\hbar$-independent, this expansion is

$$
\lambda_{i}^{\text {formal }}=y_{i}-\hbar \frac{P^{\prime}}{4 P}+\hbar^{2} y_{i} \frac{5 P^{\prime 2}-4 P P^{\prime \prime}}{32 P^{3}}+\cdots .
$$

Note that although $\lambda_{i}^{\text {formal }}$ is a formal solution of the differential equation (2.3), in writing this solution we do not have to do any integrals!

\footnotetext{
${ }^{7}$ In the important special case of $\hbar$-independent $P$, we just have $p=P$.

${ }^{8}$ In the WKB literature it is common to write the two square roots simply as $\pm \mathrm{i} \sqrt{p}$, and label the two sheets as,+- instead of $i, j$.
} 
The series $\lambda_{i}^{\text {formal }}$ in (2.6) is generally not convergent. Nevertheless, one might hope that we could interpret $\lambda_{i}^{\text {formal }}$ as an asymptotic series, and that there would be a unique actual solution $\lambda_{i}$ with $\lambda_{i} \sim \lambda_{i}^{\text {formal }}$ as $\hbar \rightarrow 0$. It turns out that the situation is more complicated. There is no $\lambda_{i}$ which has this asymptotic expansion, if $\hbar$ is allowed to approach 0 from an arbitrary direction in the complex plane. The best one can do in general is to ask for a solution $\lambda_{i}^{\vartheta}$ which has the expansion $\lambda_{i}^{\vartheta} \sim \lambda_{i}^{\text {formal }}$ as $\hbar \rightarrow 0$ while staying within a closed half-plane

$$
\mathbb{H}_{\vartheta}=\left\{\operatorname{Re}\left(\mathrm{e}^{-\mathrm{i} \vartheta} \hbar\right) \geq 0\right\} .
$$

Such a $\lambda_{i}^{\vartheta}$ actually does exist ${ }^{9}$, but only away from the $\vartheta$-Stokes curves of type $i j$, which we define next.

For simplicity we assume henceforward that $p(z)$ has only simple zeroes. Then, from each zero of $p(z)$ there emanate three trajectories along which $\int \mathrm{e}^{-\mathrm{i} \vartheta} \sqrt{-p(z)} \mathrm{d} z$ is purely real; we call these $\vartheta$-Stokes curves. The $\vartheta$-Stokes curves make up the Stokes graph $\mathcal{W}(p, \vartheta)$. Each Stokes curve is oriented away from the zero, and carries a label $i j$, determined such that $\mathrm{e}^{-\mathrm{i} \vartheta}\left(y_{i}-y_{j}\right) \mathrm{d} z$ is positive along the oriented curve. ${ }^{10}$ See Figure 1 for some examples of $\vartheta$-Stokes graphs in the case where $P(z)$ is a polynomial potential in the plane; many other such examples can be found e.g. in $[5,15]$.
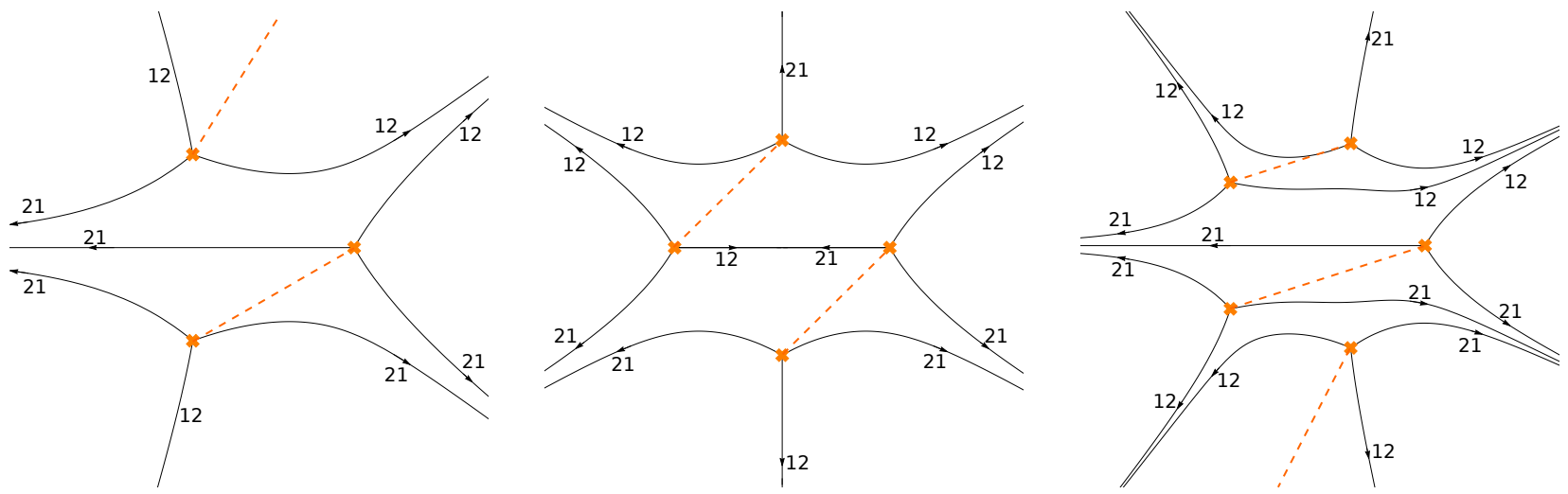

Figure 1: Examples of $\vartheta$-Stokes graphs at $\vartheta=0$, with $p(z)=z^{n}-1$, for $n=3,4,5$. The dashed lines denote branch cuts of the covering $\Sigma \rightarrow C$; the labels $i=1,2$ are swapped when we cross a cut.

As long as the domain $U$ does not contain any $\vartheta$-Stokes curve of type $i j, \lambda_{i}^{\vartheta}$ is defined on $U$ and can be integrated to give a WKB solution:

$$
\psi_{i}^{\vartheta}(z)=\exp \left(\hbar^{-1} \int_{z_{0}}^{z} \lambda_{i}^{\vartheta}(z) \mathrm{d} z\right) .
$$

\footnotetext{
${ }^{9}$ This has been a folk-theorem for some time, at least for the case of $p$ with sufficiently generic residues, and a proof has been announced by Koike-Schäfke. See [6] for an account.

${ }^{10}$ Since $y_{j}=-y_{i}$ we could also have just written that $\mathrm{e}^{-\mathrm{i} \vartheta} y_{i}$ is positive, and we could have labeled the curve just by the single index $i$ instead of the ordered pair $i j$. Our redundant-looking notation is chosen with an eye toward the generalization to higher-order equations, in Section 5 below.
} 
If $U$ does not contain any $\vartheta$-Stokes curve of either type $i j$ or $j i$, then both $\psi_{i}^{\vartheta}$ and $\psi_{j}^{\vartheta}$ exist on $U$, and give a basis of solutions of the Schrödinger equation (2.1). If $U$ does contain a $\vartheta$-Stokes curve of type $i j$, then we still get a basis of solutions on the complement of the Stokes curve, but $\psi_{i}^{\vartheta}$ jumps by a constant multiple of $\psi_{j}^{\vartheta}$ on crossing the Stokes curve.

\subsection{Abelianization}

The WKB formula (2.9) has the awkward feature that it depends on the arbitrary choice of basepoint $z_{0}$. To see the content of (2.9) more clearly, we can observe that it represents a solution of the first-order equation

$$
\left(\partial_{z}-\hbar^{-1} \lambda_{i}^{\vartheta}(z)\right) \psi_{i}^{\vartheta}(z)=0 .
$$

(2.10) is much simpler than the original equation (2.1); a lot of the complexity of (2.1) has been swallowed into solving the Riccati equation to produce $\lambda_{i}^{\vartheta}(z)$.

We interpret (2.10) as the condition that $\psi_{i}^{\vartheta}(z)$ represents a flat section of a connection $\nabla^{\mathrm{ab}, \vartheta}$ in a line bundle $\mathcal{L}$. The line bundle $\mathcal{L}$ lives not over the base $C$ but over the double cover $\Sigma$, since the function $\lambda_{i}^{\vartheta}$ depends on the sheet index $i$. The 1 -form $-\hbar^{-1} \lambda_{i}^{\vartheta} \mathrm{d} z$ represents $\nabla^{\mathrm{ab}, \vartheta}$ relative to a local trivialization of $\mathcal{L}$.

\subsection{Gluing across the Stokes graph}

Consider a $\vartheta$-Stokes curve of type ij. $\mathcal{L}$ and $\nabla^{\mathrm{ab}, \vartheta}$ naively do not extend across the lift of this $\vartheta$-Stokes curve to sheet $i$, because the solutions $\psi_{i}^{\vartheta}$ are different on the two sides. We can nevertheless extend them "by hand" by giving a gluing map which takes $\nabla^{\mathrm{ab}, \vartheta}$-flat sections on one side to $\nabla^{\mathrm{ab}, \vartheta}$-flat sections on the other, i.e. it maps $\psi_{i}^{\vartheta, L}$ to some constant multiple of $\psi_{i}^{\vartheta, R}$. There is a canonical and convenient choice: we glue $\psi_{i}^{\vartheta, L}$ to the unique multiple of $\psi_{i}^{\vartheta, R}$ which is of the form $\psi_{i}^{\vartheta, L}+\beta \psi_{j}^{\vartheta, L}$, and glue $\psi_{j}^{\vartheta, L}$ to $\psi_{j}^{\vartheta, L}$. This gluing prescription can be summarized as ${ }^{11}$

$$
\left(\begin{array}{c}
\psi_{i}^{L} \\
\psi_{j}^{L}
\end{array}\right) \mapsto\left(\begin{array}{cc}
1 & \beta \\
0 & 1
\end{array}\right)\left(\begin{array}{l}
\psi_{i}^{L} \\
\psi_{j}^{L}
\end{array}\right)=\left(\begin{array}{l}
\frac{\left[\psi_{i}^{L}, \psi_{j}^{L}\right]}{\left[\psi_{i}^{R}, \psi_{i}^{L}\right]} \psi_{i}^{R} \\
{\left[\frac{\left.\psi_{j}^{L}, \psi_{i}^{L}\right]}{\left[\psi_{j}^{R}, \psi_{i}^{L}\right]} \psi_{j}^{R}\right.}
\end{array}\right)
$$

where $\left[\psi_{1}, \psi_{2}\right]$ means the Wronskian of the two solutions.

An additional subtlety arises if a $\vartheta$-Stokes curve of type $i j$ coincides with a $\vartheta$-Stokes curve of type $j i$, as e.g. in the middle of Figure 1. (This does not occur for generic values of $\vartheta$, but it can occur for special $\vartheta$, and in many of the examples we consider in this paper we

\footnotetext{
${ }^{11}$ The gluing rule (2.11) should be regarded as a version of the "WKB connection formula."
} 
take such a special $\vartheta$.) In this case we have four distinct solutions $\psi_{i}^{\vartheta, L}, \psi_{j}^{\vartheta, L}, \psi_{i}^{\vartheta, R}, \psi_{j}^{\vartheta, R}$ on $U$, and we choose a gluing of the form

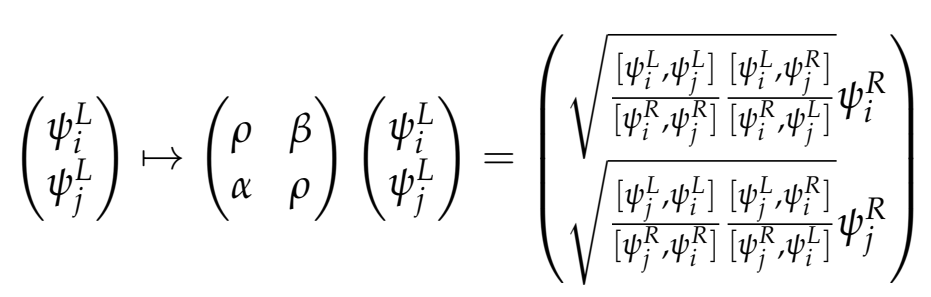

where $\rho^{2}-\alpha \beta=1$.

We must make two technical comments about the gluing rule (2.12):

- In writing (2.12) we adopted the choice that the two diagonal entries of the gluing matrix should be equal. We could alternatively have chosen e.g. that the upper left entry of the gluing matrix should be 1 , or the lower right entry should be 1 . These alternate choices also have their advantages: they arise naturally if one imagines that the two Stokes curves with labels $i j$ and $j i$ are infinitesimally displaced from one another, so that the gluing matrix arises as the product of an upper-triangular and a lower-triangular matrix. This infinitesimal displacement was used in [16, 17] and was called "resolution" of the spectral network. It appears naturally if we consider an infinitesimal perturbation of the phase $\vartheta$, either to $\vartheta+\epsilon$ or $\vartheta-\epsilon$. The choice we made in (2.12) is in some sense an average of these two resolutions, which avoids breaking symmetries.

- The gluing matrix in (2.12) is determined only up to an overall sign. To fix this ambiguity, we need to specify the branches of the square roots. For this purpose (but only for this purpose!) it is convenient to make a definite choice of the normalization of our solutions $\psi_{i}^{L / R}$, by choosing the basepoint $z_{0}$ in (2.9) to be on the Stokes curve. Then we choose the principal branch for both square roots. The motivation for this choice is that all four Wronskians appearing under the top square root asymptotically approach $2 \hbar^{-1} y_{i}$ as $\hbar \rightarrow 0$, and similarly the four Wronskians under the bottom square root approach $2 \hbar^{-1} y_{j}$, so both ratios approach 1 .

After all this gluing, we get a line bundle $\mathcal{L}$ with flat connection $\nabla^{\mathrm{ab}, \vartheta}$, defined over all of $\Sigma$ except for the branch points. It remains to consider the monodromy around the branch points. By a short calculation (see e.g. [17]), using the fact that the gluing matrices have determinant 1 , one can show that $\nabla^{\mathrm{ab}, \vartheta}$ has monodromy -1 on small loops encircling branch points. We summarize this situation by saying that $\nabla^{\mathrm{ab}, \vartheta}$ is an almost-flat connection over $\Sigma$.

\section{$2.4 \mathcal{W}$-framings}

The structure we have obtained from WKB can be encapsulated formally as follows. 
The Schrödinger equation can be interpreted as a flat connection $\nabla$ in the 1-jet bundle $J_{1}\left(K_{C}^{-\frac{1}{2}}\right)$ over $C$ : this is just the standard maneuver of replacing a second-order equation by a first-order equation with $2 \times 2$ matrix coefficients, locally written as

$$
\underbrace{\left[\partial_{z}+\hbar^{-1}\left(\begin{array}{cc}
0 & -P(z) \\
1 & 0
\end{array}\right)\right]}_{\nabla} \underbrace{\left(\begin{array}{c}
-\hbar \psi^{\prime}(z) \\
\psi(z)
\end{array}\right)}_{J(\psi)}=0 .
$$

Given a flat connection $\nabla$ and a Stokes graph $\mathcal{W}$, one can formulate the notion of a $\mathcal{W}$ abelianization of $\nabla$, as in [17] (see also [20] for a more recent and mathematical treatment). A $\mathcal{W}$-abelianization consists of:

- A flat $\mathrm{SL}(2)$-connection $\nabla$ over $C$,

- An almost-flat GL(1)-connection $\nabla^{\text {ab }}$ over $\Sigma$,

- A flat isomorphism $\iota: \pi_{*} \nabla^{\mathrm{ab}} \simeq \nabla$ away from the walls of $\mathcal{W}$ (where $\pi: \Sigma \rightarrow C$ is the projection),

obeying the constraint that, at the walls of $\mathcal{W}, \iota$ jumps by a unipotent transformation of the form (2.11) (for a wall of type $i j$ ) or (2.12) (for a wall of type $i j$ and $j i$ ).

Given the connection $\nabla$, to construct a $\mathcal{W}$-abelianization of $\nabla$ amounts to producing projective bases of $\nabla$-flat sections in the various domains of $C \backslash \mathcal{W}$, such that the relations between the bases in neighboring domains are given by matrices of the form (2.11) or (2.12). This is ultimately a linear algebra problem determined by the combinatorics of $\mathcal{W}$ and the monodromy and Stokes data of $\nabla$. For any particular $\mathcal{W}$ and $\nabla$, one can ask, how many $\mathcal{W}$-abelianizations of $\nabla$ are there? In the examples studied in [17], it turns out that there are just finitely many of them, and moreover they are in $1-1$ correspondence with some concrete extra data one can attach to $\nabla$, called $\mathcal{W}$-framings in [17]. For example,

- Suppose we consider Schrödinger equations on a Riemann surface $C$, taking $P(z)$ meromorphic with $n$ second-order poles. In this case, for generic $\vartheta$, the $\vartheta$-Stokes graph $\mathcal{W}$ is a "Fock-Goncharov" network as described in [15, 17]. A $\mathcal{W}$-framing in this case is a choice of an eigenline of the monodromy around each of the $n$ punctures. For generic $\nabla$, the monodromy at each puncture has 2 distinct eigenlines. Thus $\nabla$ admits $2^{n}$ distinct $\mathcal{W}$-framings.

- Again, suppose we consider Schrödinger equations on a Riemann surface $C$, taking $P(z)$ meromorphic with $n$ second-order poles. For special $v$, the complement of the $\vartheta$-Stokes graph $\mathcal{W}$ can include regions with the topology of an annulus. For such a $\vartheta$, a $\mathcal{W}$-framing involves additional data: a choice of an eigenline of the monodromy around each annulus. Thus $\nabla$ admits $2^{n+m}$ distinct $\mathcal{W}$-framings, where $m$ is the number of annuli.

Now we come back to WKB. The discussion of Subsection 2.1-Subsection 2.3 above can be rephrased as follows: when $\nabla$ is the flat $\mathrm{SL}(2)$-connection induced by a Schrödinger 
equation (2.1), and $\mathcal{W}$ is the Stokes graph with phase $\vartheta=\arg \hbar$, exact WKB analysis constructs a distinguished $\mathcal{W}$-abelianization of $\nabla$. This construction will be developed in more detail in [55].

It is somewhat remarkable that the WKB method automatically equips $\nabla$ with a distinguished $\mathcal{W}$-framing. In the cases above, this boils down to the statement that the local WKB solutions are automatically eigenvectors of the relevant monodromies of $\nabla$.

\subsection{Spectral coordinates and their properties}

Starting from the Schrödinger equation (2.1) and a choice of phase $\vartheta$, we have seen that exact WKB analysis gives rise to an almost-flat GL(1)-connection $\nabla^{\mathrm{ab}, \vartheta}$ over the surface $\Sigma$. In particular, given any 1-cycle $\gamma$ on $\Sigma$ there is a corresponding holonomy,

$$
\mathcal{X}_{\gamma}^{\vartheta}=\operatorname{Hol}_{\gamma} \nabla^{\mathrm{ab}, \vartheta} \in \mathbb{C}^{\times}
$$

As we have discussed in Subsection 1.3, the quantities $\mathcal{X}_{\gamma}^{\vartheta}$ have various names, among them Voros symbols, spectral coordinates, and quantum periods. They turn out to be extremely convenient for the analysis of the Schrödinger equation (2.1). Here are a few of their expected properties:

1. $\mathcal{X}_{\gamma}^{\vartheta}$ admits a complete asymptotic expansion as $\hbar \rightarrow 0$ in $\mathbb{H}_{\vartheta}$, obtained by term-byterm integration of the formal WKB series (2.6):

$$
\mathcal{X}_{\gamma}^{\vartheta} \sim \exp \left(\hbar^{-1} \oint_{\gamma} \lambda^{\text {formal }} \mathrm{d} z\right) .
$$

In particular, assuming $P(z)$ has no term of order $\hbar$, the leading asymptotic of $\mathcal{X}_{\gamma}^{\vartheta}$ is controlled by the classical period: if we define

$$
Z_{\gamma}=\oint_{\gamma} y d z
$$

then to leading order

$$
\mathcal{X}_{\gamma}^{\vartheta} \sim \pm \exp \left(\hbar^{-1} Z_{\gamma}\right)
$$

The sign \pm in (2.17) is explicitly $\exp \oint_{\gamma} \frac{1}{4} \frac{\mathrm{d} p}{p}=(-1)^{\frac{1}{2} w}$, where $w$ is the number of zeroes of $p(z)$ enclosed by the projection of $\gamma$, counted with multiplicity. ${ }^{12}$

2. $\mathcal{X}_{\gamma}^{\vartheta}$ depends on $\hbar$, on the potential $P$, and on the phase $\vartheta$. As long as the topology of the $\vartheta$-Stokes graph does not change, the dependence of $\mathcal{X}_{\gamma}^{\vartheta}$ on $\vartheta$ is trivial, while the dependence of $\mathcal{X}_{\gamma}^{\vartheta}$ on $\hbar$ and $P$ is holomorphic. There is a codimension- 1 locus in

\footnotetext{
${ }^{12}$ When $C$ is a compact Riemann surface of genus $g$, to see that $(-1)^{\frac{1}{2} w}$ does not depend on which side we call the "inside" of $\gamma$, we use the fact that a holomorphic quadratic differential has $4 g-4$ zeroes, which is divisible by 4.
} 
the $(P, \vartheta)$ parameter space where the topology of the $\vartheta$-Stokes graph does change; we call this the BPS locus. When $(P, \vartheta)$ crosses the BPS locus, the functions $\mathcal{X}_{\gamma}^{\vartheta}$ jump by a holomorphic transformation, called Stokes automorphism or Kontsevich-Soibelman transformation depending on the context. This transformation can be computed from the Stokes graph at the BPS locus. ${ }^{13}$

3. The asymptotic expansion (2.15) should hold as $\hbar \rightarrow 0$ in the half-plane $\mathbb{H}_{\vartheta}$. If $\hbar$ is exactly in the middle of the half-plane $\mathbb{H}_{\vartheta}$, i.e. if $\vartheta=\arg \hbar$, then we can make a stronger conjecture, as follows. If $(P, \vartheta)$ is not on the BPS locus, $\mathcal{X}_{\gamma}^{\vartheta}$ is the Borel sum of the asymptotic expansion (2.15) along the ray $\mathrm{e}^{\mathrm{i} \vartheta} \mathbb{R}_{+}$. If $(P, \vartheta)$ is on the BPS locus, then (2.15) may not be Borel summable along the ray $\mathrm{e}^{\mathrm{i} \vartheta} \mathbb{R}_{+}$, because of singularities of the Borel transform. In that case, our conjecture is that $\mathcal{X}_{\gamma}^{\vartheta}$ is obtained from (2.15) by Écalle's "median summation" (in the sense of [56, 57], also reviewed in [58] page 21. $)^{14}$

\subsection{Integral equations}

Finally we come to one of the most interesting properties of the spectral coordinates of families of Schrödinger operators: this is the conjecture of [26] which says that they obey integral equations as functions of $\hbar$.

There is some choice involved in writing down the equations; one has to first choose some function $\vartheta(\arg \hbar)$, subject only to the constraint that $|\vartheta(\arg \hbar)-\arg \hbar| \leq \frac{\pi}{2}$. Then one considers the specialization

$$
\mathcal{X}_{\gamma}^{\mathrm{RH}}(\hbar)=\mathcal{X}_{\gamma}^{\vartheta(\arg \hbar)}(\hbar) .
$$

$\mathcal{X}_{\gamma}^{\mathrm{RH}}$ is piecewise analytic in $\hbar$; it jumps along some rays in the $\hbar$-plane, namely those rays at which the topology of the Stokes graph $\mathcal{W}(p, \vartheta(\arg \hbar))$ jumps. We call these active rays and denote them by $r$. When $\hbar$ lies on an active ray $r$, we let $\mathcal{X}^{R H, r, \pm}(\hbar)$ denote the limit of $\mathcal{X}^{\mathrm{RH}}(\hbar)$ as arg $\hbar$ approaches the phase of $r$ from the \pm side. The conjecture of [26] says that these functions are the unique solution of a system of coupled integral equations, of the form

$$
\mathcal{X}_{\gamma}^{\mathrm{RH}}(\hbar)=\exp \left[\frac{Z_{\gamma}}{\hbar}+\frac{1}{4 \pi \mathrm{i}} \sum_{r \text { active }} \int_{r} \frac{\mathrm{d} \hbar^{\prime}}{\hbar^{\prime}} \frac{\hbar^{\prime}+\hbar}{\hbar^{\prime}-\hbar} F_{r, \gamma}\left(\mathcal{X}^{R H, r,+}\left(\hbar^{\prime}\right)\right)\right] .
$$

\footnotetext{
${ }^{13}$ In a generic situation the Stokes automorphisms which can occur are of the form $\mathcal{X}_{\mu} \rightarrow \mathcal{X}_{\mu}(1 \pm$ $\left.\mathcal{X}_{\gamma}\right)^{\Omega(\gamma)\langle\gamma, \mu\rangle}$, where $\Omega(\gamma)=+1$ for a "flip" of the Stokes graph and $\Omega(\gamma)=-2$ for a "juggle" of the Stokes graph, in the terminology of [15]. The active rays corresponding to flips are typically isolated in the $\hbar$-plane, while juggles occur at the limit of infinite sequences of flips. A general algorithm for computing the Stokes automorphism from a Stokes graph at the BPS locus is given in [16].

${ }^{14}$ This statement is sensitive to the particular gluing rule (2.12) which we chose. Had we chosen a different rule, as described below (2.12), we would expect to get instead the "lateral summation" corresponding to perturbing $\vartheta$ infinitesimally.
} 
This integral equation is similar to those appearing in the thermodynamic Bethe ansatz (TBA), and indeed (2.19) can be viewed as a generalization of the "ODE-IM correspondence" as we discussed in the introduction.

We are not aware of a completely rigorous proof of (2.19); morally the idea is that the $\mathcal{X}_{\gamma}^{\mathrm{RH}}(\hbar)$ can be uniquely characterized in terms of their analytic properties in the $\hbar$-plane, and a solution of (2.19) would necessarily have the same analytic properties, so it must be $\mathcal{X}_{\gamma}^{\mathrm{RH}}(\hbar)$. One direct argument which derives (2.19) from reasonable analytic assumptions is given in [33]. In another direction, [26] offers some reasons for optimism based on identifying (2.19) as the scaling limit of a better-behaved equation previously considered in [15]. For us, the strongest reason so far to believe (2.19) is a practical one: it has been checked to high precision in examples. So far this has been done for various simple potentials, as reported e.g. in [31, 26, 33, 59].

To formulate (2.19) completely, as we have explained, one needs to fix the choice of the function $\vartheta(\arg \hbar)$. One canonical possibility is to take

$$
\vartheta(\arg \hbar)=\arg \hbar .
$$

The resulting functions $\mathcal{X}_{\gamma}^{\mathrm{RH}}(\hbar)$ are obtained by making WKB analysis for each $\hbar$ using the Stokes graph adapted to the phase $\vartheta=\arg \hbar$. This choice makes the functions $F_{r, \gamma}$ relatively simple, at the cost that there may be many active rays (even infinitely many), and one has to consider all possible $\vartheta$-Stokes graphs. See Figure 2.

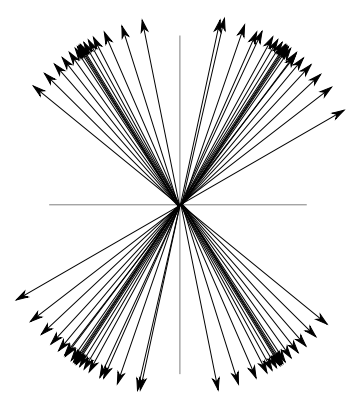

Figure 2: A sample picture of what the active rays in the $\hbar$-plane can look like. There are in general infinitely many such rays, which can accumulate at discrete phases (as shown here) or even be dense in part or all of the $\hbar$-plane.

Another natural choice is to take $\vartheta(\arg \hbar)$ to be piecewise constant; this has the effect of dividing the plane into sectors (of opening angle $\leq \pi$ ) and collapsing all the active rays in each sector $S_{i}$ onto a single "aggregated" ray $r_{i}$. In this case the aggregated functions $F_{r_{i}, \gamma}$ contain equivalent information to all of the functions $F_{r, \gamma}$ for $r \subset S_{i}$.

In any case, to determine concretely the functions attached to the active rays, one can use the relation

$$
F_{r, \gamma}\left(\mathcal{X}_{\gamma}^{R H, r,+}\right)=\log \left(\mathcal{X}_{\gamma}^{R H, r,+} / \mathcal{X}_{\gamma}^{R H, r,-}\right),
$$

if one knows the spectral coordinate systems $\mathcal{X}_{\gamma}^{\vartheta}$ for $\vartheta$ on both sides of the active ray $r$. 
The most extreme possibility is to divide the plane into just two sectors, by fixing a phase $\alpha$ and defining

$$
\vartheta(\arg \hbar)= \begin{cases}\alpha & \text { for } \hbar \in \mathbb{H}_{\alpha \prime} \\ \alpha+\pi & \text { for } \hbar \in \mathbb{H}_{\alpha+\pi}\end{cases}
$$

In this case there are just 2 active rays $r$, and we only have to consider two Stokes graphs,
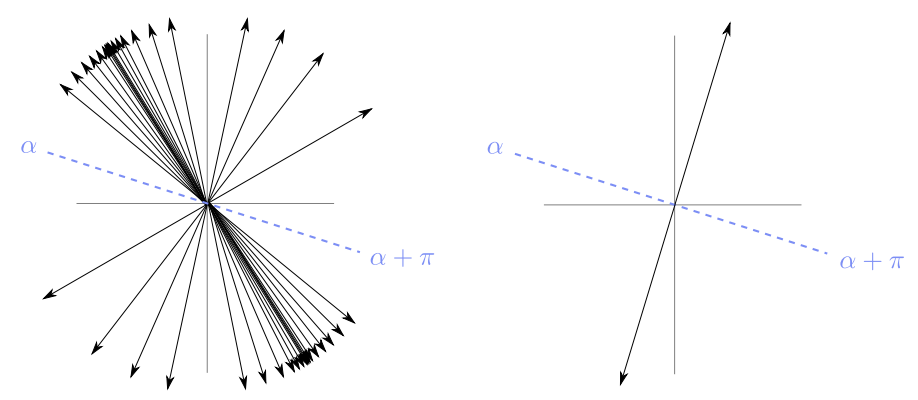

Figure 3: Collapsing infinitely many active rays down to 2 by making the choice (2.22). Each active ray on the right carries functions $F_{r, \gamma}$ which should be thought of as containing the same information as all the $F_{r, \gamma}$ in the corresponding half-plane on the left.

the $\alpha$-Stokes graph and the $(\alpha+\pi)$-Stokes graph. These two Stokes graphs are moreover identical except for an overall relabeling of all the Stokes lines, $i j \rightarrow j i$. The function $F_{r, \gamma}$ on each of the 2 active rays contains equivalent information to the "spectrum generator" discussed in [15]. ${ }^{15}$ When the $\alpha$-Stokes graph is of "Fock-Goncharov type," the spectrum generator has been determined in [15]; these results were used in [26] to give several explicit examples of integral equations (2.19).

\section{Exact WKB for Schrödinger operators with cubic poten- tial}

The WKB method and exact WKB method have been explored rather thoroughly in the case of a Schrödinger equation in the plane with polynomial potential. For the WKB method two important references are [61, 62]; for exact WKB see e.g. the pioneering works $[1,3,63,64]$, and [5] for a clear recent treatment.

In this section we quickly touch on the very simplest example of this sort, the Schrödinger equation

$$
\left[\hbar^{2} \partial_{z}^{2}+\left(z^{3}-u\right)\right] \psi(z)=0
$$

for a constant $u \in \mathbb{C}$. This is an instance of (2.1) with cubic potential

$$
P(z)=z^{3}-u \text {. }
$$

\footnotetext{
${ }^{15}$ In the cluster algebra literature this object is called the "Donaldson-Thomas transformation" or "DT transformation" following [60].
} 


\subsection{A Stokes graph}

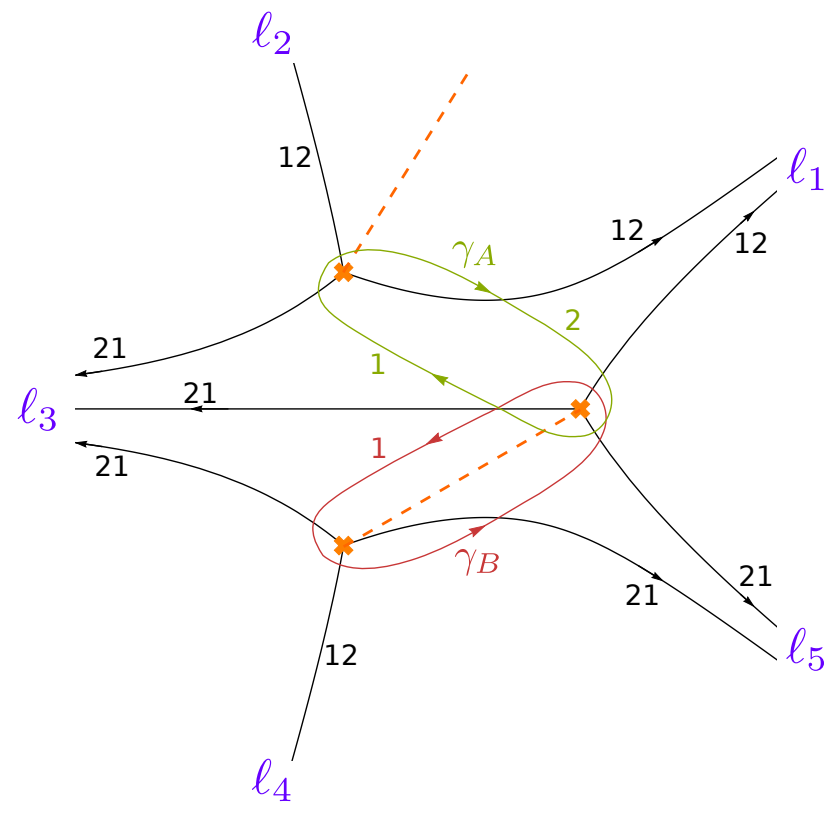

Figure 4: $\vartheta$-Stokes graph for the Schrödinger equation with cubic potential (3.1), at the phase $\vartheta=0$, and $u=1$. Two 1-cycles $\gamma_{A}, \gamma_{B}$ on $\Sigma$ are also shown. Dashed orange segments denote branch cuts; on crossing a cut, the sheet labels are exchanged $1 \leftrightarrow 2$. Orange crosses denote the turning points, zeroes of $p(z)=z^{3}-1$. The singularity at $z=\infty$ is not shown.

Suppose we fix $u=1$ and $\vartheta=0$. Then the $\vartheta$-Stokes graph is shown in Figure 4 .

This graph divides the plane up into 7 domains. As we have reviewed in Section 2, there are canonical local solutions $\lambda_{i}^{\vartheta}$ of the Riccati equation in each of these domains, and from these local solutions we can build local WKB solutions $\psi_{i}^{\vartheta}$ of (2.1) - or more invariantly, we can build an almost-flat GL(1)-connection $\nabla^{\mathrm{ab}, \vartheta}$ over the spectral curve $\Sigma=\left\{y^{2}+z^{3}-1=0\right\}$. The connection $\nabla^{\mathrm{ab}, \vartheta}$ abelianizes the $\operatorname{SL}(2)$-connection $\nabla$ in the $z$-plane associated to (3.1).

\subsection{The spectral coordinates}

Let $\mathcal{X}_{A}\left(\right.$ resp. $\left.\mathcal{X}_{B}\right)$ denote the monodromy of $\nabla^{\mathrm{ab}, \vartheta}$ along the cycle $\gamma_{A}\left(\right.$ resp. $\left.\gamma_{B}\right)$ in Figure 4. The Stokes graph of Figure 4 is an example of a Fock-Goncharov network in the sense of [17], and $\mathcal{X}_{A}, \mathcal{X}_{B}$ are Fock-Goncharov coordinates of the flat connection $\nabla$. Let us explain this more concretely.

We first consider the local WKB solutions in each domain. These turn out to have a simple and concrete characterization, as follows.

Let $\ell_{n}(n=1, \ldots, 5)$ denote the ray with phase $\frac{2 \pi}{5}\left(n+\frac{1}{2}\right)$. When $\hbar \in \mathbb{R}_{+}$, for each $n$ there exists a solution $\psi_{n}^{\mathrm{sm}}$ such that $\psi_{n}^{\mathrm{sm}}$ decays exponentially as $z \rightarrow \infty$ along $\ell_{n}$. This $\psi_{n}^{\mathrm{sm}}$ is unique up to scalar multiple. Now let $U$ be one of the domains in the complement 
of the Stokes graph. $U$ has two infinite "ends" which approach two of the five rays $\ell_{n}$. As $z$ approaches $\ell_{n}$, the WKB solution $\psi_{j}^{\vartheta}$ is exponentially decaying, where $i j$ is the label on the $\vartheta$-Stokes curves asymptotic to $\ell_{n}{ }^{16}$ Thus up to scalar multiple $\psi_{j}^{\vartheta}$ is equal to $\psi_{n}^{\mathrm{sm}}$.

Now that we understand the local WKB solutions, we can use them to compute the spectral coordinates. They turn out to be cross-ratios of the $\psi_{n}^{\mathrm{sm}}$, as follows (see Appendix A for the computation):

$$
\mathcal{X}_{A}=\frac{\left[\psi_{3}^{\mathrm{sm}}, \psi_{2}^{\mathrm{sm}}\right]}{\left[\psi_{1}^{\mathrm{sm}}, \psi_{2}^{\mathrm{sm}}\right]} \frac{\left[\psi_{1}^{\mathrm{sm}}, \psi_{5}^{\mathrm{sm}}\right]}{\left[\psi_{3}^{\mathrm{sm}}, \psi_{5}^{\mathrm{sm}}\right]}, \quad \mathcal{X}_{B}=\frac{\left[\psi_{3}^{\mathrm{sm}}, \psi_{4}^{\mathrm{sm}}\right]}{\left[\psi_{5}^{\mathrm{sm}}, \psi_{4}^{\mathrm{sm}}\right]} \frac{\left[\psi_{5}^{\mathrm{sm}}, \psi_{1}^{\mathrm{sm}}\right]}{\left[\psi_{3}^{\mathrm{sm}}, \psi_{1}^{\mathrm{sm}}\right]}
$$

As we promised above, these are Fock-Goncharov coordinates (or "complexified shear coordinates") of the connection $\nabla$, in the sense of [21].

\subsection{Analytic continuation}

In our description of $\mathcal{X}_{A}$ and $\mathcal{X}_{B}$ above we used the conditions $u=1$ and $\hbar \in \mathbb{R}_{+}$. It is interesting to consider the question of analytic continuation of these functions in $u$ and $\hbar$. For this purpose a simple approach is to just start from the final formulas (3.3) and try to continue them directly. The resulting analytic structure is very simple:

- First, as we vary $u$, the $z \rightarrow \infty$ asymptotic behavior of the equation (3.1) does not change; for each $u$ we still have 5 decaying solutions $\psi_{n}^{\mathrm{sm}}$, now depending on $u$. Since the equation (3.1) depends holomorphically on $u$, so do its decaying solutions. Thus the formula (3.3) defines single-valued analytic functions $\left(\mathcal{X}_{A}, \mathcal{X}_{B}\right)$ of $u \in \mathbb{C}$.

These functions may have poles, because for general $u$ there is nothing preventing $\psi_{n}^{\mathrm{sm}}$ and $\psi_{n^{\prime}}^{\mathrm{sm}}$ from coinciding, as long as $n$ and $n^{\prime}$ are not consecutive. Indeed, numerically one finds a discrete sequence of points $u=u_{1}, u_{2}, \ldots$ where $\mathcal{X}_{A}$ has a simple pole ( $\psi_{3}^{\mathrm{sm}}$ and $\psi_{5}^{\mathrm{sm}}$ become proportional) and conjugate points $u=u_{1}^{*}, u_{2}^{*}, \ldots$ where $\mathcal{X}_{B}$ has a simple pole ( $\psi_{3}^{\mathrm{sm}}$ and $\psi_{1}^{\mathrm{sm}}$ become proportional). These poles can be thought of as "bound states" for the equation (3.1) along a complex contour asymptotic to $\ell_{n}$ and $\ell_{n^{\prime}}$. The $u_{i}$ lie on the ray $\arg u=-\frac{4}{5} \pi$ (but this is not trivial to see: it was proven in [65].)

- Second, we can consider varying $\hbar$. This leads to a slightly subtler analytic structure. If we vary $\arg \hbar$ by an amount $\beta$, the distinguished rays $\ell_{n}$ where we impose the exponential decay condition rotate counterclockwise in the plane by an angle $\frac{2}{5} \beta$. It follows that, when we go clockwise once around the singularity at $\hbar=0$, the $\psi_{n}^{\mathrm{sm}}$ are permuted by $n \mapsto n+2(\bmod 5)$; this transforms $\left(\mathcal{X}_{A}, \mathcal{X}_{B}\right)$ by

$$
\left(\mathcal{X}_{A}, \mathcal{X}_{B}\right) \mapsto\left(\mathcal{X}_{B}^{-1}\left(1-\mathcal{X}_{A}^{-1}\right)^{-1}, \mathcal{X}_{A}\right)
$$

\footnotetext{
${ }^{16}$ This follows from the realization of $\lambda_{j}^{\vartheta}$ as the Borel summation of the WKB series, which implies that $\lambda_{j}^{\vartheta} \mathrm{d} z$ is negative along $\ell_{n}$, since every term of the series has this property.
} 
Thus the maximal analytic continuation of the functions $\left(\mathcal{X}_{A}, \mathcal{X}_{B}\right)$ is defined on a 5 -fold cover of the punctured plane $\hbar \in \mathbb{C}^{\times}$.

In fact, the continuations in $u$ and $\hbar$ are not unrelated: the continued functions actually depend only on the combination $u^{\prime}=u / \hbar^{\frac{6}{5}}$, as one sees by dividing (3.1) by $\hbar^{\frac{6}{5}}$ and then taking $z \mapsto \hbar^{2} z$.

Note that the monodromy (3.4) acts by a symplectomorphism preserving the form $\omega=\mathrm{d} \log \mathcal{X}_{A} \wedge \mathrm{d} \log \mathcal{X}_{B}$. This is a consistency check of the general story: $\mathcal{X}_{A}$ and $\mathcal{X}_{B}$ are local Darboux coordinates on a moduli space of SL(2)-connections with irregular singularity at $z=\infty$.

We emphasize that the analytic continuation of $\left(\mathcal{X}_{A}, \mathcal{X}_{B}\right)$ which we have been discussing in this section is not given directly by WKB analysis; to make the WKB analysis directly at a given $(u, \hbar)$ would require us to consider a different Stokes graph and spectral curve for each $(u, \hbar)$. This would necessarily lead to single-valued functions of $(u, \hbar)$, but ones which are only piecewise analytic, jumping when the Stokes graph jumps. These are the functions which we called $\mathcal{X}_{\gamma}^{\mathrm{RH}}$ above; we will discuss them more in Subsection 3.5 below.

\subsection{The regular pentagon}

One case worthy of special notice is the case $u=0$, where the potential $P(z)$ degenerates to the pure cubic, $P(z)=z^{3}$. At this point the equation (3.1) acquires an extra $\mathbb{Z} / 5 \mathbb{Z}$ symmetry which acts by $z \rightarrow \mathrm{e}^{2 \pi \mathrm{i} / 5} z$, and thus cyclically permutes the five rays $\ell_{n}$. From this symmetry it follows that $\left(\mathcal{X}_{A}, \mathcal{X}_{B}\right)$ is a fixed point of the monodromy (3.4), which implies

$$
\mathcal{X}_{A}=\mathcal{X}_{B}=x, \quad x^{2}-x-1=0 .
$$

Numerically we find that the relevant solution of this quadratic is ${ }^{17}$

$$
\mathcal{X}_{A}=\mathcal{X}_{B}=\frac{1-\sqrt{5}}{2} \text {. }
$$

Since $\mathcal{X}_{A}$ and $\mathcal{X}_{B}$ depend only on $u^{\prime}=u / \hbar^{\frac{6}{5}}$, it follows that this fixed point also governs the $\hbar \rightarrow \infty$ behavior for any constant $u$.

${ }^{17}$ The reader might wonder: what about the other solution, where $\mathcal{X}_{A}=\mathcal{X}_{B}=\frac{1+\sqrt{5}}{2}$ ? That one turns out to be associated to a Schrödinger equation with singular potential, $P(z)=z^{3}-\frac{3}{4} \frac{\hbar^{2}}{z^{2}}$. The specific coefficient $-\frac{3}{4} \hbar^{2}$ here ensures that the singularity at $z=0$ is only an "apparent singularity," with trivial monodromy; thus this equation is still associated to a flat connection $\nabla$ in the plane, and all our discussion of abelianization applies equally well to this case. Moreover this equation still has the $\mathbb{Z} / 5 \mathbb{Z}$ symmetry (because the two terms $z^{3}$ and $1 / z^{2}$ differ by a factor $z^{5}$ ), and numerically one checks that it has $\mathcal{X}_{A}=\mathcal{X}_{B}=\frac{1+\sqrt{5}}{2}$. We thank Dylan Allegretti and Tom Bridgeland for several enlightening conversations about Schrödinger equations with apparent singularities. 


\subsection{Integral equations for spectral coordinates}

Identifying the cross-ratios (3.3) as the spectral coordinates coming from WKB implies that they should have all the properties discussed in Subsection 2.5-Subsection 2.6. In particular, when they are extended to functions $\mathcal{X}_{\gamma}^{\mathrm{RH}}(\hbar)$ as in Subsection 2.6, they should obey an integral equation of the form (2.19).

We make the canonical choice (2.20). Then one direct way to identify the active rays is to use a computer to draw the $\vartheta$-Stokes graphs for various phases $\vartheta$; the active rays are at the phases where the $\vartheta$-Stokes graph jumps. It turns out that there are 6 such rays, as shown in Figure 5. ${ }^{18}$ Each of these rays $r$ has an associated class $\mu \in H_{1}(\Sigma, \mathbb{Z})$, and the function $F_{r, \gamma}$ is

$$
F_{r, \gamma}(\mathcal{X})=\langle\gamma, \mu\rangle \log \left(1-\mathcal{X}_{\mu}\right)
$$

where $\langle\cdot, \cdot\rangle$ is the intersection pairing on $H_{1}(\Sigma, \mathbb{Z})$.

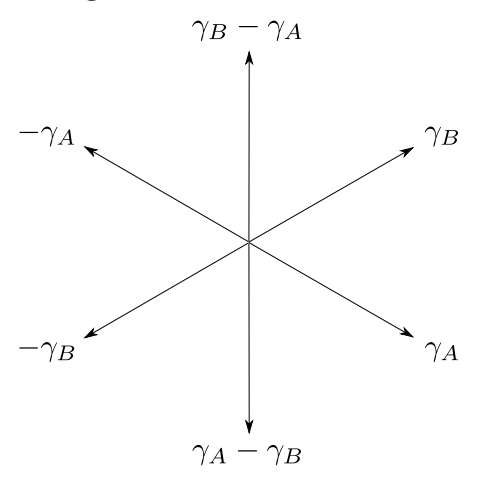

Figure 5: The 6 active rays in the $\hbar$-plane, each labeled by its charge $\mu \in H_{1}(\Sigma, \mathbb{Z})$. These rays divide the $\hbar$-plane into 6 regions. Each region is characterized by a different topology for the $\vartheta$-Stokes graph, where $\vartheta=\arg \hbar$.

In this case one can make a direct numerical test of the integral equation (2.19). Namely, on the one hand we can solve (2.19) by numerical iteration, on the other hand we can determine $\mathcal{X}_{\gamma}^{\mathrm{RH}}(\hbar)$ directly by numerical integration of the Schrödinger equation in the complex plane. The two computations agree very well. To give one concrete example, by direct numerical integration we obtain the estimates

$$
\begin{aligned}
& \mathcal{X}_{A}(\hbar=2+\mathrm{i}) \approx-0.230042356-0.324912345 \mathrm{i}, \\
& \mathcal{X}_{B}(\hbar=2+\mathrm{i}) \approx-0.288795812+0.476012574 \mathrm{i},
\end{aligned}
$$

and each of these agrees with the result obtained from the integral equation (2.19), to the precision given. Many similar computations for polynomial potentials have been made before, with similarly good numerical agreement, e.g. already in [30] and more recently $[26,33,59]$. The appearance of the fixed point (3.6) at the $\hbar \rightarrow \infty$ limit was already noticed in the very early TBA work [67].

\footnotetext{
${ }^{18}$ This corresponds to the well known BPS spectrum of the $\left(A_{1}, A_{2}\right)$ Argyres-Douglas field theory in its "maximal chamber," discussed e.g. in [66, 15].
} 


\section{Exact WKB for the Mathieu equation}

Now let us recall how exact WKB analysis is applied to the Mathieu equation:

$$
\left[-\hbar^{2} \partial_{x}^{2}+2 \cos (x)-2 E\right] \psi(x)=0 .
$$

WKB analysis of this equation has been studied extensively; a review we found particularly helpful is [68], which covers many topics we will not touch here. For other treatments of exact WKB for this equation see e.g. [37, 69, 40, 42], and more broadly [70, 71, 72].

\subsection{Exponential coordinate}

Making the replacements

$$
z=\mathrm{e}^{\mathrm{i} x}, \quad \tilde{\psi}(z)=(\mathrm{i} z)^{\frac{1}{2}} \psi(x)
$$

transforms (4.1) into an equation defined over $\mathbb{C P}^{1}$, with irregular singularities at $z=0$ and $z=\infty$ :

$$
\left[\hbar^{2} \partial_{z}^{2}+P(z)\right] \tilde{\psi}(z)=0, \quad P(z)=\frac{1}{z^{3}}-\frac{2 E-\frac{1}{4} \hbar^{2}}{z^{2}}+\frac{1}{z} .
$$

In what follows we will usually use the formulation (4.3).

\subsection{A simple Stokes graph}

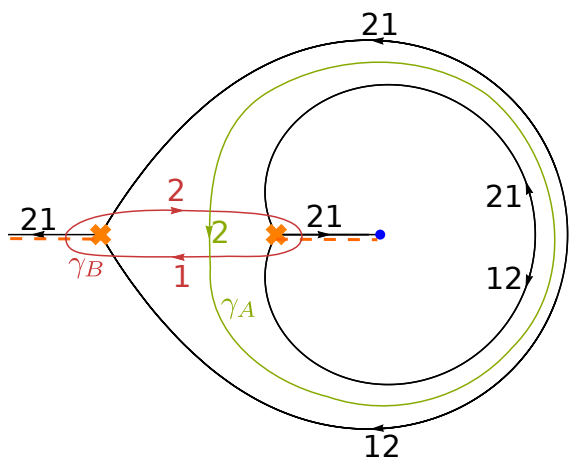

Figure 6: $\vartheta$-Stokes graph for the Mathieu equation, at the phase $\vartheta=0$, and $E=-\frac{9}{8}$. Two 1cycles $\gamma_{A}, \gamma_{B}$ on $\Sigma$ are also shown. Dashed orange segments denote branch cuts; on crossing a cut, the sheet labels are exchanged $1 \leftrightarrow 2$. Orange crosses denote the turning points, zeroes of $P(z)$. The blue dot represents the singularity at $z=0$; the singularity at $z=\infty$ is not shown.

We begin with real $E<-1$ and $\vartheta=0$. The $\vartheta$-Stokes graph is as shown in Figure 6 . The Stokes curves divide the plane into 3 open domains: a simply connected domain near $z=0$, another near $z=\infty$, and an annulus containing $z=1$. 


\subsection{The spectral coordinates}

Let $\mathcal{X}_{A}$ (resp. $\mathcal{X}_{B}$ ) denote the monodromy of $\nabla^{\mathrm{ab}, \vartheta}$ along the cycle $\gamma_{A}$ (resp. $\gamma_{B}$ ) in Figure 6. This Stokes graph is an example of a Fenchel-Nielsen network in the sense of [17], and $\mathcal{X}_{A}, \mathcal{X}_{B}$ are exponentiated Fenchel-Nielsen coordinates of the flat connection $\nabla$. Let us explain this more concretely.

We first consider local WKB solutions in each of the three domains.

- Let $\psi_{i}$ be the local WKB solutions near $z=0 . \psi_{1}$ can be characterized as a solution which exponentially decays as $z \rightarrow 0$ along the negative- $z$ ray, similarly to what we saw in Subsection 3.2.

- Let $\psi_{i}^{\prime}$ be the local WKB solutions near $z=\infty . \psi_{1}^{\prime}$ can be characterized as a solution which exponentially decays as $z \rightarrow \infty$ along the negative- $z$ ray.

- Let $\psi_{i}^{\prime \prime}$ be the local WKB solutions in some simply connected domain of the intermediate annulus. These can be characterized as eigenvectors of the counterclockwise monodromy $M$ of $\nabla$. At $E<-1$ and $\hbar \in \mathbb{R}_{+}$the eigenvalues of $M$ are real and negative, and we let $\mu$ denote the eigenvalue which has $|\mu|<1$; then $\psi_{1}^{\prime \prime}$ is the eigenvector with eigenvalue $\mu^{-1}$, while $\psi_{2}^{\prime \prime}$ is the one with eigenvalue $\mu$.

With the local WKB solutions understood, we can compute the spectral coordinates:

- $\mathcal{X}_{A}$ is the smaller eigenvalue of monodromy of $\nabla$,

$$
\mathcal{X}_{A}=\mu \text {. }
$$

Indeed, the representative $\gamma_{A}$ in Figure 6 does not cross any Stokes curves, so the eigenvalue of monodromy of $\nabla^{\mathrm{ab}}$ on sheet 2 agrees with the eigenvalue of monodromy of $\nabla$ acting on $\psi_{2}^{\prime \prime}$, which is $\mu$. This is an exponentiated complexified Fenchel-Nielsen length coordinate, in the sense of $[24,17]$.

- $\mathcal{X}_{B}$ can be given in terms of Wronskians of the local WKB solutions in the three domains (see Appendix A for the computation):

$$
\mathcal{X}_{B}=\frac{\left[\psi_{1}, \psi_{2}^{\prime \prime}\right]}{\left[\psi_{1}, \psi_{1}^{\prime \prime}\right]} \frac{\left[\psi_{1}^{\prime}, \psi_{1}^{\prime \prime}\right]}{\left[\psi_{1}^{\prime}, \psi_{2}^{\prime \prime}\right]}
$$

(In computing these Wronskians we have to evolve all the solutions to a common domain, which we do along the negative- $z$ ray.) This is an exponentiated complexified Fenchel-Nielsen twist coordinate, in the sense of [24, 17].

\subsection{Application: bound states}

Now let us see one application of the spectral coordinates. We return to the original Mathieu equation (4.1) and make the substitution $x=\mathrm{i} x^{\prime}+\pi$ with $x^{\prime}$ real. Then (4.1) 
becomes the modified Mathieu equation,

$$
\left[-\hbar^{2} \partial_{x^{\prime}}^{2}+2 \cosh \left(x^{\prime}\right)+2 E\right] \psi\left(x^{\prime}\right)=0 .
$$

This is a Schrödinger equation with potential $V\left(x^{\prime}\right)=\cosh x^{\prime}$, for which we can formulate the usual bound state problem, i.e. we look for $E$ such that there exists an $L^{2}$ solution of (4.6). Such a solution exists only for countably many $E=E_{1}, E_{2}, \ldots$ With our sign conventions $E$ is minus the usual energy, so all $E_{n}<-1$.

The condition for existence of a bound state is that $\psi_{1}$ is proportional to $\psi_{1}^{\prime}$. Substituting this condition in (4.5) gives simply

$$
\mathcal{X}_{B}=1 .
$$

This is known as the "exact quantization condition" for the modified Mathieu bound states, discussed frequently in the literature, e.g. [43, 69, 37].

To give some indication of how (4.7) can be used in practice, let us consider the leading term of the asymptotic expansion,

$$
\mathcal{X}_{B} \approx-\exp \left(Z_{B} / \hbar\right)
$$

When $E<-1$ we have $Z_{B} \in \mathrm{i} \mathbb{R}_{-}$, and recall that $\hbar>0$; thus this leading approximation says that solutions of (4.7) will be found when

$$
Z_{B} \approx 2 \pi \mathrm{i}\left(n+\frac{1}{2}\right) \hbar .
$$

To understand (4.9) more explicitly, we can expand $Z_{B}$ at large negative $E$ : one finds $Z_{B}(E) \approx-4 \mathrm{i} \sqrt{-2 E} \log (-E)$. Thus, for large negative $E$ and small $\hbar$, the desired bound states are approximately at

$$
\sqrt{-E} \log (-E) \approx \frac{\pi}{2 \sqrt{2}}\left(n+\frac{1}{2}\right) \hbar .
$$

One can improve this estimate by including higher terms - either in the WKB expansion of $\mathcal{X}_{B}$ in powers of $\hbar$, or in the expansion of $Z_{B}(E)$ in inverse powers of $E$. We will not explore these improvements here.

\subsection{Analytic continuation}

So far we have considered the spectral coordinates $\mathcal{X}_{A}$ and $\mathcal{X}_{B}$ for $E<-1, \hbar>0$, built using the exact WKB method. It is also interesting to consider the analytic continuation of these coordinates to complex parameters.

To build this analytic continuation, we will build a $\mathcal{W}$-abelianization of $\nabla$ which varies holomorphically with $(E, \hbar)$. Said otherwise, we will build local solutions $\psi_{i}, \psi_{i}^{\prime}, \psi_{i}^{\prime \prime}$ which 
fit into a $\mathcal{W}$-abelianization and vary holomorphically with $(E, \hbar)$. For general $(E, \hbar)$ they will not necessarily be given by any kind of WKB analysis.

The local solutions $\psi_{i}^{\prime \prime}$ must be eigenvectors of the monodromy $M$ : to decide which one will be $\psi_{1}^{\prime \prime}$ and which will be $\psi_{2}^{\prime \prime}$, we just require that our choice is continuously connected to the choice we got from WKB at $E<-1, \hbar>0$. This gives a nice analytic continuation along any path in $(E, \hbar)$ space, except at the codimension-1 locus where the eigenvalues of $M$ coincide. Around this locus we have an order-2 monodromy exchanging $\psi_{1}^{\prime \prime} \leftrightarrow \psi_{2}^{\prime \prime}$.

At our initial locus $(E<-1, \hbar>0), \psi_{1}$ can be characterized by the property of exponential decay as $z \rightarrow 0$ along the negative real axis. As we vary $(E, \hbar)$ we can define $\psi_{1}$ by a similar condition, except that the negative real axis has to be replaced by a different path, which asymptotically has $z \rightarrow 0$ with $\arg z=2 \arg \hbar+\pi$. Similar comments apply to $\psi_{1}^{\prime}$ except that we use a path with $z \rightarrow \infty$ and $\arg z=-2 \arg \hbar+\pi$. This gives a nice analytic continuation of $\psi_{1}$ and $\psi_{1}^{\prime}$ along any path in $(E, \hbar)$ space which avoids $\hbar=0$. Now we have to consider the possibility of monodromy around $\hbar=0$. As arg $\hbar$ is continuously increased by $2 \pi$, our paths into $z=0$ and $z=\infty$ wind around twice, in opposite directions. The result is that as we go counterclockwise around $\hbar=0$ we have an infinite-order monodromy acting by $\psi_{1} \mapsto M^{-2} \psi_{1}, \psi_{1}^{\prime} \mapsto M^{2} \psi_{1}^{\prime}$.

(We might also wonder whether the eigenvectors $\psi_{i}^{\prime \prime}$ of $M$ are exchanged as $\hbar$ goes around 0; this cannot occur, since the Mathieu equation depends only on $\hbar^{2}$, so the monodromy around $\hbar=0$ is the square of an order-2 element, hence the identity.)

Using (4.4) and (4.5), the analytic structure of $\mathcal{X}_{A}$ and $\mathcal{X}_{B}$ follows from that of $\psi_{i}, \psi_{i}^{\prime}$, $\psi_{i}^{\prime \prime}$; we have unrestricted analytic continuation in $(E, \hbar)$, except that:

- Going around $\hbar=0$ counterclockwise we have the infinite-order monodromy

$$
\left(\mathcal{X}_{A}, \mathcal{X}_{B}\right) \mapsto\left(\mathcal{X}_{A}, \mathcal{X}_{A}^{8} \mathcal{X}_{B}\right)
$$

- Around the locus in $(E, \hbar)$ space where the eigenvalues of $M$ coincide, we have the order-2 monodromy

$$
\left(\mathcal{X}_{A}, \mathcal{X}_{B}\right) \mapsto\left(\mathcal{X}_{A}^{-1}, \mathcal{X}_{B}^{-1}\right) .
$$

Note that both of these monodromies act by symplectomorphisms preserving the form $\omega=\mathrm{d} \log \mathcal{X}_{A} \wedge \mathrm{d} \log \mathcal{X}_{B}$. This is a consistency check of the general story: $\mathcal{X}_{A}$ and $\mathcal{X}_{B}$ are local Darboux coordinates on the moduli space of SL(2)-connections.

\subsection{Integral equations for spectral coordinates}

As we have discussed in Subsection 2.6, one of the most interesting properties of spectral coordinates for families of Schrödinger equations is that they conjecturally obey integral equations of the form (2.19).

In the case of the Mathieu equation, integral equations for spectral coordinates were considered in [26]. There the function $\vartheta(\arg \hbar)$ was chosen in the form (2.22), with $\alpha$ a 
generic phase, collapsing all the active rays onto two aggregated rays. In this case the Stokes graphs which appear are of Fock-Goncharov type in the terminology of [17], and the $\mathcal{X}_{\gamma}$ are Fock-Goncharov coordinates. This example is thus qualitatively similar to the one we considered in Subsection 3.5 above, though the details are more intricate. ${ }^{19}$

In this section we try something different: we try to find integral equations obeyed by the complexified Fenchel-Nielsen coordinates. For this purpose we choose the very nongeneric phase $\alpha=0$, so that the aggregated rays are the positive and negative imaginary axes. See Figure 7.
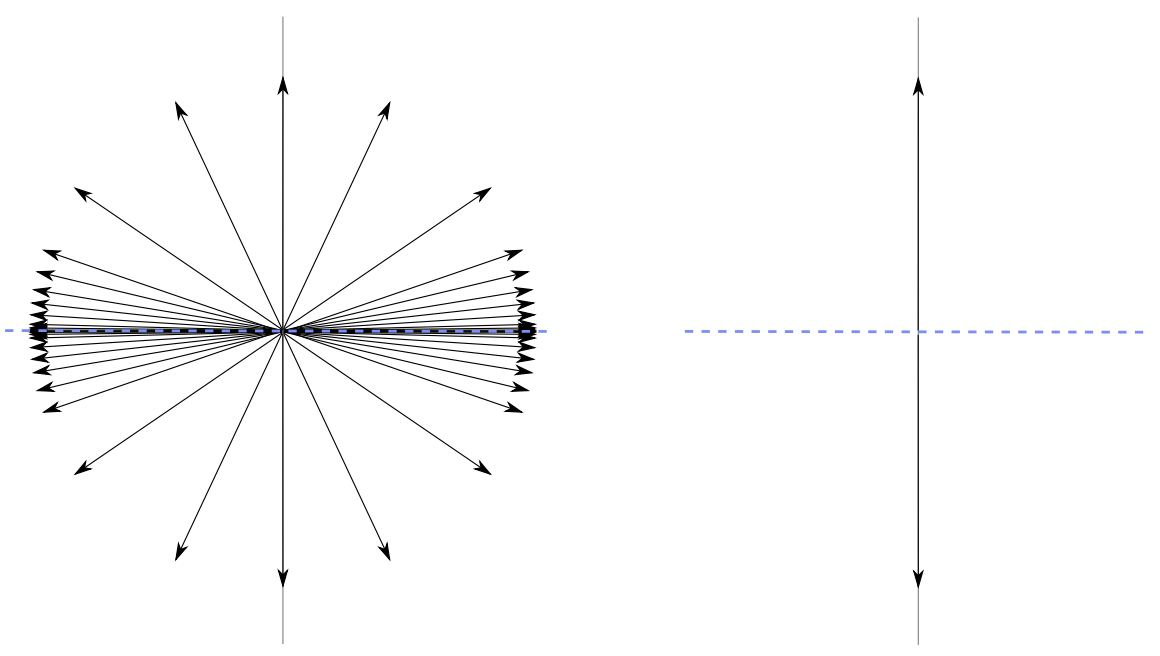

Figure 7: Collapsing the infinitely many active rays down to 2 by making the choice (2.22) with $\alpha=0$. Each active ray on the right carries functions $F_{r, \gamma}$ which should be thought of as containing the same information as all the $F_{r, \gamma}$ in the corresponding half-plane on the left. This is a particularly thorny case because the active rays on the left accumulate at the boundary of the half-planes.

Then, according to the recipe of Subsection 2.6, the functions $\mathcal{X}_{\gamma}^{\mathrm{RH}}$ are:

$$
\mathcal{X}_{\gamma}^{\mathrm{RH}}(\hbar)= \begin{cases}\mathcal{X}_{\gamma}^{\vartheta=0}(\hbar) & \text { for } \operatorname{Re} \hbar>0, \\ \mathcal{X}_{\gamma}^{\vartheta=\pi}(\hbar) & \text { for } \operatorname{Re} \hbar<0 .\end{cases}
$$

Now, to construct the functions $F_{r, \gamma}$ appearing in the integral equation (2.19), we need to understand the discontinuity of $\mathcal{X}_{\gamma}^{\mathrm{RH}}$ across the imaginary axis. It turns out that this discontinuity has a more complicated form than those we have previously considered: $\mathcal{X}_{\gamma}^{\mathrm{RH}}$ is continuous along some segments of the axis, and discontinuous along other segments. Correspondingly the functions $F_{r, \gamma}$ must be zero on some segments and nonzero on others, so in particular they cannot be holomorphic functions of $\mathcal{X}_{\gamma}$. This feature is related to the

\footnotetext{
${ }^{19}$ In particular, it seems to be harder to find a solution of the integral equations (2.19) directly by iteration in this case. Instead one can start with a slightly different system of integral equations, those used in [73]; these one can solve by iteration; then one can take the limit $R \rightarrow 0, \zeta \rightarrow 0, \hbar=R / \zeta$, to get solutions of (2.19).
} 
fact that each $r$ aggregates contributions from infinitely many rays which accumulate at the boundary of the half-plane, as shown in Figure 7.

We can work out the discontinuities of the functions $\mathcal{X}_{\gamma}$ by keeping track of their symmetry properties. First, we have

$$
\mathcal{X}_{\gamma}^{\vartheta=\pi}(-\hbar)=\mathcal{X}_{\gamma}^{\vartheta=0}(\hbar)^{-1} .
$$

Second, $\mathcal{X}_{A}(\hbar)$ is real for $\hbar>0$, which implies the reality property $\mathcal{X}_{A}(\bar{\hbar})=\overline{\mathcal{X}_{A}(\hbar)}$. Combining this with (4.14) we get

$$
\mathcal{X}_{A}^{\mathrm{RH}}(-\bar{\hbar})={\overline{\mathcal{X}_{A}^{\mathrm{RH}}(\hbar)}}^{-1} .
$$

It follows that the discontinuity of $\mathcal{X}_{A}^{\mathrm{RH}}$ at the imaginary axis is

$$
\mathcal{X}_{A}^{\mathrm{RH}} \mapsto \overline{\mathcal{X}}_{A}^{\mathrm{RH}}{ }^{-1}=\mathcal{X}_{A}^{\mathrm{RH}} \times\left|\mathcal{X}_{A}^{\mathrm{RH}}\right|^{-2} .
$$

For $\mathcal{X}_{B}^{\mathrm{RH}}$ it is similar except that the reality property has an extra sign, $\mathcal{X}_{B}(\bar{\hbar})={\overline{\mathcal{X}_{B}(\hbar)}}^{-1}$, giving

$$
\mathcal{X}_{B}^{\mathrm{RH}}(-\bar{\hbar})=\overline{\mathcal{X}_{B}^{\mathrm{RH}}(\hbar)} .
$$

Thus the discontinuity of $\mathcal{X}_{B}^{\mathrm{RH}}$ is

$$
\mathcal{X}_{B}^{\mathrm{RH}} \mapsto \overline{\mathcal{X}_{B}^{\mathrm{RH}}}=\mathcal{X}_{B}^{\mathrm{RH}} \times \frac{\overline{\mathcal{X}_{B}^{\mathrm{RH}}}}{\mathcal{X}_{B}^{\mathrm{RH}}} .
$$

Substituting these discontinuities into the general form (2.19) using (2.21), we get integral equations which are most naturally written directly in terms of $x_{\gamma}=\log \mathcal{X}_{\gamma}$ :

$$
\begin{aligned}
& x_{A}^{\mathrm{RH}}(\hbar)=\frac{Z_{A}}{\hbar}+\frac{1}{2 \pi \mathrm{i}} \int_{0}^{\mathrm{i} \infty} \mathrm{d} \hbar^{\prime}\left(\frac{2 \hbar}{\hbar^{\prime 2}-\hbar^{2}}\right)\left(-2 \operatorname{Re} x_{A}^{\mathrm{RH}}\left(\hbar^{\prime}+0\right)\right), \\
& x_{B}^{\mathrm{RH}}(\hbar)=\frac{Z_{B}}{\hbar}+\frac{1}{2 \pi \mathrm{i}} \int_{0}^{\mathrm{i} \infty} \mathrm{d} \hbar^{\prime}\left(\frac{2 \hbar}{\hbar^{\prime 2}-\hbar^{2}}\right)\left(-2 \mathrm{i} \operatorname{Im} x_{B}^{\mathrm{RH}}\left(\hbar^{\prime}+0\right)\right) .
\end{aligned}
$$

Numerical experimentation gives us some confidence that (4.19), (4.20) do indeed hold.

These equations by themselves do not fully characterize $x_{A}^{\mathrm{RH}}$ and $x_{B}^{\mathrm{RH}}$; to see this it is enough to observe that they admit the "trivial" solutions $x_{\gamma}^{\mathrm{RH}}(\hbar)=\frac{Z_{\gamma}}{\hbar}$. This is a bit disappointing when we compare to simpler examples like that of Subsection 3.5, where it is believed that the integral equations do characterize the spectral coordinates, and even give a useful way of computing them. One hope remains; the actual functions $x_{\gamma}^{\mathrm{RH}}$ obey one more important condition: for $\hbar \in \pm \mathrm{i} \mathbb{R}$, the quantity $x_{A}^{\mathrm{RH}} \pm 2 x_{B}^{\mathrm{RH}}$ is always either real or pure imaginary. It would be interesting to know whether this property together with (4.19), (4.20) is enough to determine the functions $x_{\gamma}^{\mathrm{RH}}$. 


\subsection{Another Stokes graph}

To get good information about the region $E>-1$ from $W K B$, we switch to considering the $\vartheta$-Stokes graphs relevant for that region. There are two possibilities, depending on whether $E \in(-1,1)$ or $E>1$. Here we will just discuss $E>1$. Then the $\vartheta$-Stokes graph for $\vartheta=0$ is shown in Figure 8 .

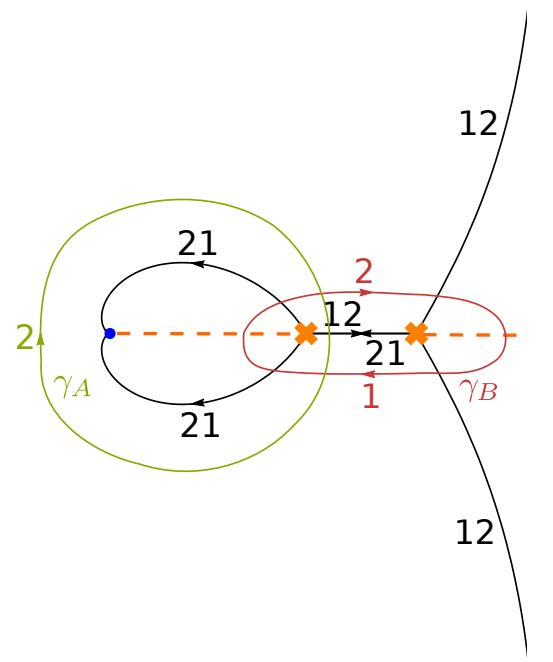

Figure 8: $\vartheta$-Stokes graph for the Mathieu equation, at the phase $\vartheta=0$, and $E=\frac{41}{40}$. All notation is as in Figure 6.

\subsection{The spectral coordinates}

Let $\psi$ denote the unique solution of (4.3) which decays exponentially as $z \rightarrow 0$ along the negative real axis, $\psi^{\prime}$ the unique solution which decays exponentially as $z \rightarrow \infty$ along the negative real axis, and $M$ the operator of counterclockwise monodromy around $z=0$. Then we have (see Appendix A)

$$
\mathcal{X}_{A}= \pm \sqrt{\frac{\left[\psi, M \psi^{\prime}\right]}{\left[M \psi, \psi^{\prime}\right]}}, \quad \mathcal{X}_{B}=\frac{[\psi, M \psi]\left[\psi^{\prime}, M \psi^{\prime}\right]}{\left[\psi, \psi^{\prime}\right]^{2}} .
$$

In particular, unlike Subsection 4.3, here there is no spectral coordinate which is equal to an eigenvalue of $M$. Nevertheless, we can express the trace of the monodromy in terms of spectral coordinates:

$$
\operatorname{Tr} M=\left(\mathcal{X}_{A}+\mathcal{X}_{A}^{-1}\right) \sqrt{1-\mathcal{X}_{B}}
$$

One quick way to see (4.22) is to write $M$ relative to the basis $\left(\psi, \psi^{\prime}\right)$ as a matrix $\left(\begin{array}{ll}a & b \\ c & d\end{array}\right)$; then (4.22) becomes

$$
a+d= \pm\left(\sqrt{\frac{d}{a}}+\sqrt{\frac{a}{d}}\right)(\sqrt{1+b c})
$$


which indeed holds, using the fact that $a d-b c=1$. To fix the sign we use the facts that, at small $\hbar, \sqrt{1-\mathcal{X}_{B}}$ is exponentially close to 1 , and $\mathcal{X}_{A}$ is exponentially close to an eigenvalue of $M$.

\subsection{Application: quasiperiodic solutions}

Now we consider the application of these spectral coordinates to another classical spectral problem. If we consider $x$ to be a real variable, then (4.1) is a Schrödinger equation with periodic potential, $V(x)=\cos x$. The standard analysis of such equations involves fixing $v \in \mathbb{R} / 2 \pi \mathbb{Z}$ (quasimomentum) and looking at solutions obeying the quasiperiodic boundary condition

$$
\psi(x+2 \pi)=\mathrm{e}^{\mathrm{i} v} \psi(x) .
$$

For fixed $v$, solutions of (4.1), (4.24) exist only at a countable set of energies $E=E_{1}, E_{2}, \ldots$, with all $E_{n}>-1$. These can be thought of as analogues of the bound state energies for a confining potential on the real line.

Using (4.22) we can rewrite the quasiperiodicity condition (4.24) as ${ }^{20}$

$$
\left(\mathcal{X}_{A}+\mathcal{X}_{A}^{-1}\right) \sqrt{1-\mathcal{X}_{B}}=-2 \cos v .
$$

This is another example of an "exact quantization condition" in the terminology of exact WKB (however, we have not found precisely (4.25) in the literature.)

When $E>1$ we have $Z_{B} \in \mathbb{R}_{-}$, and the leading WKB asymptotic $\mathcal{X}_{B} \approx-\exp \left(Z_{B} / \hbar\right)$, so the factor $\sqrt{1-\mathcal{X}_{B}}$ in (4.25) gives an exponentially small correction. As a first approach we could try neglecting this correction. Then (4.25) reduces to

$$
\mathcal{X}_{A} \approx-\mathrm{e}^{ \pm \mathrm{i} v}
$$

To derive concrete predictions from (4.26) we can use the WKB series for $\mathcal{X}_{A}$. For example, suppose we take the leading asymptotic $\mathcal{X}_{A} \approx-\exp \left(Z_{A} / \hbar\right)$, and further take large $E$, so that $Z_{A} \approx 2 \pi \mathrm{i} \sqrt{2 E}$ : then we get

$$
\mathrm{e}^{2 \pi \mathrm{i} \sqrt{2 E} / \hbar} \approx \mathrm{e}^{ \pm \mathrm{i} v}
$$

i.e.

$$
E \approx \frac{\hbar^{2}}{2}\left(n \pm \frac{v}{2 \pi}\right)^{2}
$$

This is indeed the leading behavior of the energies at large $E$ and small $\hbar$; in fact, in this limit we can approximate the quasiperiodic solutions with given $v$ simply by the free-particle wavefunctions, $\psi(x) \approx \mathrm{e}^{\mathrm{i}( \pm n+v / 2 \pi) x}$.

\footnotetext{
${ }^{20}$ The minus sign on the right side in (4.25) arises because of the square-root cut in the transformation (4.2).
} 
To improve the accuracy one could include subleading terms in the WKB series of $\mathcal{X}_{A}$; this gives perturbative corrections in a power series in $\hbar$. Likewise one could take more terms in the expansion of $Z_{A}$ around large $E$. This would modify the relation between $E$ and $(n, v)$ but preserve the basic feature that for every $E$ there is some corresponding $(n, v)$ with $v$ real. Indeed, even if we used the exact $\mathcal{X}_{A}$ in (4.26), we would still find that for every $E$ there is a corresponding $(n, v)$ with $v$ real; this follows from the fact that $\left|\mathcal{X}_{A}\right|=1$ for all large enough real $E$, a consequence of (4.21).

Now, let us consider the nonperturbative correction $\sqrt{1-\mathcal{X}_{B}}$ in (4.25). This has a qualitatively new effect: when $\mathcal{X}_{A}(E)$ is close to \pm 1 , the LHS of (4.25) can have absolute value larger than 2. For such an $E$ there is no solution to (4.25) for any real $v$; the eigenvalues of the monodromy become complex. This is the well-known phenomenon of "gaps" in the Mathieu spectrum.

It is known that the width of the gaps is exponentially suppressed by $\frac{1}{2} Z_{B} / \hbar$; see e.g. [68] for discussion and references on this point. ${ }^{21}$ Let us see how to recover this fact from the exact quantization condition (4.25). Taking $\cos v=-1$, expanding $\mathcal{X}_{A}=1+\delta \mathcal{X}_{A}$ and taking $\mathcal{X}_{B}$ small, (4.25) gives

$$
\left(2+\left(\delta \mathcal{X}_{A}\right)^{2}\right)\left(1-\frac{1}{2} \mathcal{X}_{B}\right) \approx 2,
$$

i.e. the leading-order displacement of $\mathcal{X}_{A}$ from the gap center is

$$
\delta \mathcal{X}_{A} \approx \pm \sqrt{\mathcal{X}_{B}}
$$

and thus the leading-order displacement of $E$ from the gap center is

$$
\delta E \approx \pm \frac{\sqrt{\mathcal{X}_{B}}}{\partial \mathcal{X}_{A} / \partial E}
$$

If we further take the leading $\hbar \rightarrow 0$ asymptotics of $\mathcal{X}_{A}$ and $\mathcal{X}_{B}$, this becomes ${ }^{22}$

$$
\delta E \approx \pm \hbar\left(\mathrm{i} \frac{\partial Z_{A}}{\partial E}\right)^{-1} \exp \left(\frac{1}{2 \hbar} Z_{B}\right)
$$

One could try to go beyond this leading-order estimate using the full $\hbar$ expansions of $\mathcal{X}_{A}$ and $\mathcal{X}_{B}$. It would be interesting to know whether in this way one can recover the more detailed results on the gap widths explained in [68].

\footnotetext{
${ }^{21}$ In this context the quantity $\frac{1}{2} Z_{B}$ might be called a "1-instanton action" since it corresponds to the change in the exponent of a WKB solution upon integrating along a one-way path from one branch point to another, as opposed to $Z_{B}$ which is the integral over the round-trip path $\gamma_{B}$.

${ }^{22}$ As a check against blunders, we numerically computed the width of a few of the gaps and obtained reasonable agreement: for example, when $\hbar=0.2$, there is a gap extending from $E_{-} \approx 1.3836418$ to $E_{+} \approx$ 1.3838946 , which thus has $\delta E=\frac{1}{2}\left(E_{+}-E_{-}\right) \approx 0.0001264$, while the estimate (4.32) gives $\delta E \approx 0.0001278$.
} 


\section{Exact WKB for higher order equations}

So far we have been discussing order 2 differential equations (2.1). We now move to the case of order 3 equations, involving two meromorphic "potentials" $P_{2}$ and $P_{3}$ :

$$
\left[\partial_{z}^{3}+\hbar^{-2} P_{2} \partial_{z}+\left(\hbar^{-3} P_{3}+\frac{1}{2} \hbar^{-2} P_{2}^{\prime}\right)\right] \psi(z)=0
$$

The equation (5.1) can be given a global meaning on a Riemann surface $C$ with a complex projective structure, as with (2.1) above; in this case $\psi(z)$ is a section of $K_{C}^{-1}, P_{2}(z)$ is a meromorphic quadratic differential, and $P_{3}(z)$ is a meromorphic cubic differential.

In this section we explain how the exact WKB method is expected to extend to equations of the form (5.1). In this situation there are no rigorous results yet, but there is a reasonable conjectural picture. (The same picture is expected to work for equations of any order $K \geq 2$; we stick to $K=3$ to be concrete, and because our main example has $K=3$.) Some numerical evidence supporting this conjectural picture in special cases has been obtained in $[18,59]$. We will give more numerical evidence in the case of the $T_{3}$ equation in Subsection 6.7 and Subsection 6.10 below.

All the formal structures in the story are parallel to the order 2 case, so this section is organized in parallel to Section 2 , and we will be very brief.

\subsection{WKB solutions}

WKB solutions of (5.1) are solutions of the form

$$
\psi(z)=\exp \left(\hbar^{-1} \int_{z_{0}}^{z} \lambda(z) \mathrm{d} z\right)
$$

where now $\lambda$ must obey a higher analogue of the Riccati equation (2.3),

$$
\lambda^{3}+3 \hbar \lambda \partial_{z} \lambda+\hbar^{2} \partial_{z}^{2} \lambda+P_{2} \lambda+P_{3}+\frac{1}{2} \hbar P_{2}^{\prime}=0
$$

One again constructs WKB solutions $\lambda_{i}^{\text {formal }}$ as power series in $\hbar$. As before, one meets an ambiguity at order $\hbar^{0}$; this ambiguity is resolved by choosing a sheet $i$ of the 3-fold covering

$$
\Sigma=\left\{y^{3}+p_{2}(z) y+p_{3}(z)=0\right\} .
$$

Now the conjectural picture is that, as in the order 2 case, there exist actual solutions of (5.3) which have the asymptotic behavior $\lambda_{i}^{\vartheta} \sim \lambda_{i}^{\text {formal }}$ in the half-plane $\mathbb{H}_{\vartheta}$, away from $\vartheta$-Stokes curves.

The $\vartheta$-Stokes curves carry labels $i j$. Along a $\vartheta$-Stokes curve of type $i j, \mathrm{e}^{-\mathrm{i} \vartheta}\left(y_{i}-y_{j}\right) \mathrm{d} z$ is real and positive. We make the simplifying assumption that all branch points of $\Sigma$ are simple branch points, i.e. only two $y_{i}$ collide at a time. For the construction of the $\vartheta$-Stokes 
graph in this case see [16]. One key new feature of the higher-order case, first discovered in [74] and further investigated in e.g. [75, 14, 16], is that Stokes curves of type $i k$ can be born from intersections of Stokes curves of types $i j$ and $j k$. See Figure 9 for an example.

The local solution $\lambda_{i}^{\vartheta}$ of (5.3) is supposed to exist away from $\vartheta$-Stokes curves of type $i j$, as in the order 2 case. On crossing a $\vartheta$-Stokes curve of type $i j$, we conjecture that the local WKB solution $\psi_{i}^{\vartheta}$ jumps by a constant multiple of $\psi_{j}^{\vartheta} .{ }^{23}$

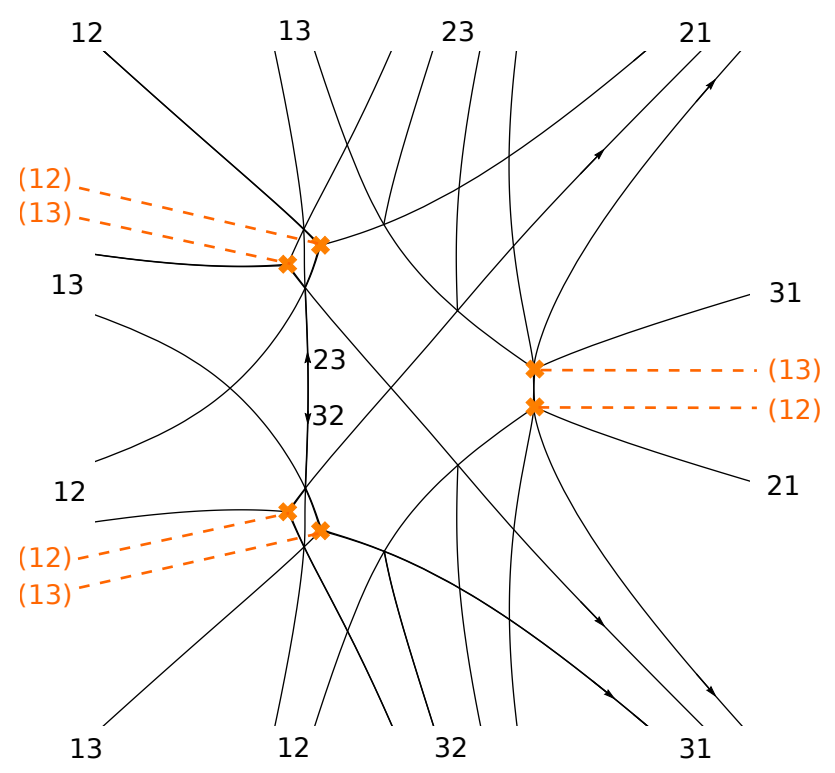

Figure 9: An example of a $\vartheta$-Stokes graph at $\vartheta=0$, with $p_{2}(z)=1$ and $p_{3}(z)=z^{3}-1$.

\subsection{Abelianization}

As in the order 2 case, the WKB solutions of (5.1) can be thought of as solutions of a first-order equation over $\Sigma$, built using the $\lambda_{i}^{\vartheta}$. Thus, as before, exact WKB analysis of (5.1) leads to a line bundle $\mathcal{L}$ with almost-flat connection $\nabla^{\mathrm{ab}, \vartheta}$ over $\Sigma$, away from the $\vartheta$-Stokes curves.

\footnotetext{
${ }^{23}$ Some evidence for this conjecture has been given in [76]. We thank Kohei Iwaki for pointing out this reference.
} 


\subsection{Gluing across the Stokes graph}

Also as before, we can glue $\mathcal{L}$ and $\nabla^{\mathrm{ab}, \vartheta}$ across the $\vartheta$-Stokes curves. At a $\vartheta$-Stokes curve of type $i j$ the gluing takes the form (cf. (2.11))

$$
\left(\begin{array}{c}
\psi_{i}^{L} \\
\psi_{j}^{L} \\
\psi_{k}^{L}
\end{array}\right) \mapsto\left(\begin{array}{ccc}
1 & \beta & 0 \\
0 & 1 & 0 \\
0 & 0 & 1
\end{array}\right)\left(\begin{array}{l}
\psi_{i}^{L} \\
\psi_{j}^{L} \\
\psi_{k}^{L}
\end{array}\right)=\left(\begin{array}{l}
\frac{\left[\psi_{i}^{L}, \psi_{j}^{L}, \psi_{k}^{L}\right]}{\left[\psi_{i}^{R}, \psi_{i}^{L}, \psi_{k}^{L}\right]} \psi_{i}^{R} \\
\frac{\left[\psi_{j}^{L}, \psi_{k}^{L}, \psi_{i}^{L}\right]}{\left[\psi_{j}^{R}, \psi_{k}^{L}, \psi_{i}^{L}\right]} \psi_{j}^{R} \\
{\left[\frac{\left[\psi_{k}^{L}, \psi_{i}^{L}, \psi_{j}^{L}\right]}{\left[\psi_{k}^{R}, \psi_{i}^{L}, \psi_{j}^{L}\right]} \psi_{k}^{R}\right.}
\end{array}\right) .
$$

If $\vartheta$-Stokes curves of type $i j$ and $j i$ coincide, we choose a gluing of the form (cf. (2.12) $)^{24}$

$$
\left(\begin{array}{c}
\psi_{i}^{L} \\
\psi_{j}^{L} \\
\psi_{k}^{L}
\end{array}\right) \mapsto\left(\begin{array}{ccc}
\rho & \beta & 0 \\
\alpha & \rho & 0 \\
0 & 0 & 1
\end{array}\right)\left(\begin{array}{c}
\psi_{i}^{L} \\
\psi_{j}^{L} \\
\psi_{k}^{L}
\end{array}\right)=\left(\begin{array}{c}
\sqrt{\frac{\left[\psi_{i}^{L}, \psi_{j}^{L}, \psi_{k}^{L}\right]\left[\psi_{i}^{L}, \psi_{j}^{R}, \psi_{k}^{L}\right]}{\left[\psi_{i}^{R}, \psi_{j}^{R}, \psi_{k}^{L}\right]\left[\psi_{i}^{R}, \psi_{j}^{L}, \psi_{k}^{L}\right]}} \psi_{i}^{R} \\
\sqrt{\frac{\left[\psi_{j}^{L}, \psi_{i}^{L}, \psi_{k}^{L}\right]\left[\psi_{j}^{L}, \psi_{i}^{R}, \psi_{k}^{L}\right]}{\left[\psi_{j}^{R}, \psi_{i}^{R}, \psi_{k}^{L}\right]\left[\psi_{j}^{R}, \psi_{i}^{L}, \psi_{k}^{L}\right]}} \psi_{j}^{R} \\
\frac{\left[\psi_{k}^{L}, \psi_{i}^{L}, \psi_{j}^{L}\right]}{\left[\psi_{k}^{R}, \psi_{i}^{L}, \psi_{j}^{L}\right]} \psi_{k}^{R}
\end{array}\right),
$$

with $\rho^{2}-\alpha \beta=1$. (The branches of the square roots are fixed as was done above in the $K=2$ case.) By this process we obtain a line bundle $\mathcal{L}$ with almost-flat connection $\nabla^{\mathrm{ab}, \vartheta}$ over $\Sigma$.

\subsection{Spectral coordinates}

Finally we can introduce higher-order versions of the spectral coordinates: as before, these are defined by

$$
\mathcal{X}_{\gamma}^{\vartheta}=\operatorname{Hol}_{\gamma} \nabla^{\mathrm{ab}, \vartheta} \in \mathbb{C}^{\times} .
$$

The $\mathcal{X}_{\gamma}^{\vartheta}$ are expected to have all the same formal properties as in the order 2 case, discussed in Subsection 2.5-Subsection 2.6; we will not repeat those here.

\section{Exact $W K B$ for the $T_{3}$ equation}

Now we consider a specific instance of (5.1), a third-order ODE over $\mathbb{C P}^{1}$ with three regular singularities. By convention we place the singularities at $\left\{1, \omega, \omega^{2}\right\}$ where $\omega=$

\footnotetext{
${ }^{24}$ As in the order 2 case (see Subsection 2.3) this is not the only possible choice, but it is the most invariant choice.
} 


$$
\begin{aligned}
& \mathrm{e}^{2 \pi \mathrm{i} / 3.25} \\
& \quad\left[\partial_{z}^{3}+\hbar^{-2} P_{2} \partial_{z}+\left(\hbar^{-3} P_{3}+\frac{1}{2} \hbar^{-2} P_{2}^{\prime}\right)\right] \psi(z)=0, \quad P_{2}=\frac{9 \hbar^{2} z}{\left(z^{3}-1\right)^{2}}, \quad P_{3}=\frac{u}{\left(z^{3}-1\right)^{2}} .
\end{aligned}
$$

We call (6.1) the $T_{3}$ equation. This equation actually does not depend on $u$ and $\hbar$ separately, but only on the combination $u^{\prime}=u / \hbar^{3} \in \mathbb{C}$.

\subsection{A simple Stokes graph}

The $\vartheta$-Stokes graphs for the $T_{3}$ equation were investigated in [34]. It was found there that the topology of the $\vartheta$-Stokes graph depends on the phase of the quantity $w=\mathrm{e}^{-3 \mathrm{i} \vartheta} u$. For a generic phase of $w$, it seems likely that the Stokes graph is "wild" - in particular, that it is dense at least in some parts of $\mathbb{C P}^{1}$. WKB analysis involving such a wild Stokes graph may ultimately be very interesting, but we are not brave enough to try it today. ${ }^{26}$ Instead, we focus on the non-generic situation where the Stokes graph is compact; this happens for a countable set of phases of $w$. We will not rederive the form of the Stokes graphs here, but simply lift them from [34].

The simplest Stokes graph arises when $w$ is real; to be completely concrete, we take $u>0$ and $\vartheta=0$. See Figure 10 .

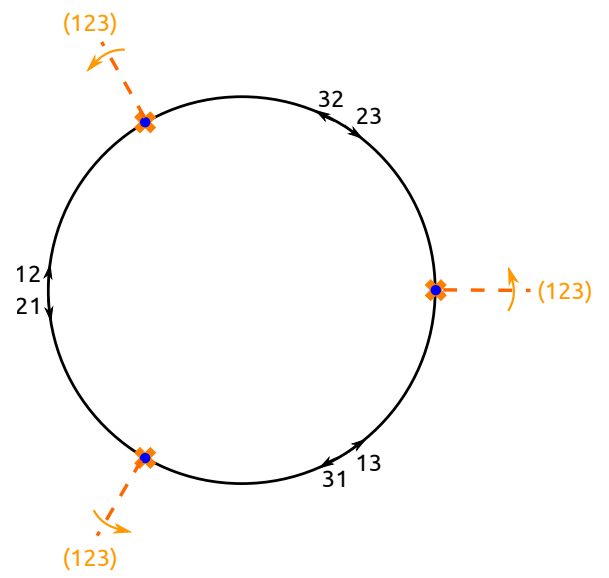

Figure 10: The $\vartheta$-Stokes graph for the $T_{3}$ equation, in case $u>0$ and $\vartheta=0$. The three branch cuts emanating from the singularities meet at $z=\infty$.

Applying the higher-order exact $\mathrm{WKB}$ method is expected to produce a $\mathcal{W}$-abelianization of the $T_{3}$ equation. Thus, we should begin by understanding concretely what this means.

We explained in Subsection 2.4 that $\mathcal{W}$-abelianizations of a meromorphic Schrödinger equation with second-order poles are in 1-1 correspondence with discrete data called $\mathcal{W}$ framings, and the choice of a $\mathcal{W}$-framing amounts to choosing one of the two eigenvectors

\footnotetext{
${ }^{25}$ Our conventions here differ from those of [34] by the replacement $u \rightarrow-u$. Sorry.

${ }^{26}$ In the order 2 case, some of the necessary analytic technology for dealing with wild Stokes graphs is developed in [77]. It would be exciting to develop the higher-rank analogue of this.
} 
of the monodromy around each singularity and each cylinder. In the case of the $T_{3}$ equation, we will have a formally similar story, except that the linear-algebra problem one has to solve to find $\mathcal{W}$-abelianizations is more intricate: it does not just correspond to choosing eigenvectors of monodromy matrices.

\subsection{The abelianization problem for the $T_{3}$ equation}

The local solutions of (6.1) in a neighborhood of $z=0$ form a 3-dimensional vector space $V$. In Figure 11 we show three cycles $A, B, C$ on $\mathbb{C P}^{1} \backslash\left\{1, \omega, \omega^{2}\right\}$, beginning and ending at $z=0$.

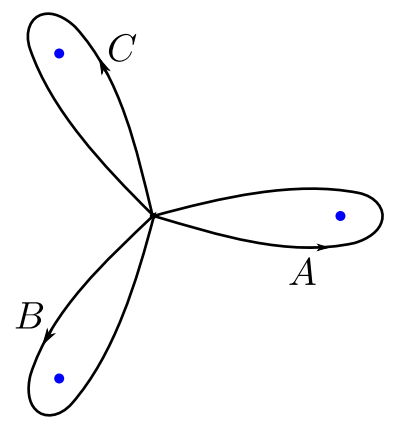

Figure 11: Three cycles on $\mathbb{C P}^{1} \backslash\left\{1, \omega, \omega^{2}\right\}$.

Let A, B, C denote the maps $V \rightarrow V$ induced by monodromy of (6.1) around these three cycles. Note they satisfy

$$
\mathrm{ABC}=1 .
$$

We say a basis $\left(\psi_{1}, \psi_{2}, \psi_{3}\right)$ of $V$ is in special position if the following conditions are satisfied:

$$
\begin{aligned}
& \mathbf{C} \psi_{1}, \mathbf{B}^{-1} \psi_{2} \in\left\langle\psi_{1}, \psi_{2}\right\rangle, \\
& \mathbf{A} \psi_{2}, \mathbf{C}^{-1} \psi_{3} \in\left\langle\psi_{2}, \psi_{3}\right\rangle, \\
& \mathbf{B} \psi_{3}, \mathbf{A}^{-1} \psi_{1} \in\left\langle\psi_{3}, \psi_{1}\right\rangle .
\end{aligned}
$$

A concrete way to think about the special-position constraint is that relative to the basis $\left(\psi_{1}, \psi_{2}, \psi_{3}\right)$ the monodromy endomorphisms must have zeroes in specific places:

$$
\begin{array}{rr}
\mathbf{A}=\left(\begin{array}{lll}
* & 0 & * \\
* & * & * \\
* & * & *
\end{array}\right), \quad \mathbf{B}=\left(\begin{array}{lll}
* & * & * \\
* & * & 0 \\
* & * & *
\end{array}\right), \quad \mathbf{C}=\left(\begin{array}{lll}
* & * & * \\
* & * & * \\
0 & * & *
\end{array}\right), \\
\mathbf{A}^{-1}=\left(\begin{array}{lll}
* & * & * \\
0 & * & * \\
* & * & *
\end{array}\right), & \mathbf{B}^{-1}=\left(\begin{array}{lll}
* & * & * \\
* & * & * \\
* & 0 & *
\end{array}\right), \quad \mathbf{C}^{-1}=\left(\begin{array}{lll}
* & * & 0 \\
* & * & * \\
* & * & *
\end{array}\right) .
\end{array}
$$

The special-position constraint is invariant under rescalings of the vectors $\left(\psi_{1}, \psi_{2}, \psi_{3}\right)$ : it depends only on the projective basis of $V$, which we can view as a 3-tuple of points in the projective space $\mathbb{P}(V) \simeq \mathbb{C P}^{2}$. 
The point of this definition is the following, proven in Appendix A: $\mathcal{W}$-abelianizations for the $T_{3}$ equation are in 1-1 correspondence with projective bases $\left(\psi_{1}, \psi_{2}, \psi_{3}\right)$ of $V$ in special position.

Now the question arises: how can we enumerate the possible projective bases of $V$ in special position? Note that (6.4) imposes 6 conditions on the basis, so a naive dimension count would suggest that bases obeying these constraints should occur discretely. To enumerate them precisely is a problem of algebraic geometry, which we address in Subsection 6.3 below. The outcome is that when $\mathbf{A}, \mathbf{B}, \mathbf{C}$ are unipotent and generic enough there are " $4+\infty$ " projective bases in special position: 4 occurring discretely plus a 1-parameter family.

\subsection{Projective bases in special position}

In this section we consider the following question. Suppose given unipotent endomorphisms $\mathbf{A}, \mathbf{B}, \mathbf{C}$ of a 3-dimensional complex vector space $V$, obeying $\mathbf{A B C}=\mathbf{1}$. Assume that $\mathbf{A}, \mathbf{B}, \mathbf{C}$ are in general position; concretely this means that each of $\mathbf{A}, \mathbf{B}, \mathbf{C}$ preserves a unique complete flag, and these flags are in general position. How do we enumerate the projective bases of $V$ in special position?

We begin with an observation. Let $\left\langle e_{\mathbf{A}}\right\rangle$ denote the eigenline of $\mathbf{A}$ and similarly for B, C. Suppose that $\left(\psi_{1}, \psi_{2}, \psi_{3}\right)$ is a projective basis in special position. Assume that $\left\langle\psi_{1}\right\rangle \neq\left\langle e_{\mathbf{C}}\right\rangle$. Then $\left\langle\psi_{1}, \mathbf{C} \psi_{1}\right\rangle$ is a plane, and (6.3a) says this plane contains both $\psi_{2}$ and $\mathbf{B}^{-1} \psi_{2}$. Equivalently, we have

$$
\psi_{2} \in\left\langle\psi_{1}, \mathbf{C} \psi_{1}\right\rangle, \quad \psi_{2} \in \mathbf{B}\left\langle\psi_{1}, \mathbf{C} \psi_{1}\right\rangle .
$$

Now, these two planes are not equal (if they were, then (6.3c) would show that this plane also contains $\psi_{3}$, contradicting the linear independence of the $\psi_{i}$.) Since both contain $\psi_{2}$, their intersection must be precisely $\left\langle\psi_{2}\right\rangle$ :

$$
\left\langle\psi_{2}\right\rangle=\left\langle\psi_{1}, \mathbf{C} \psi_{1}\right\rangle \cap \mathbf{B}\left\langle\psi_{1}, \mathbf{C} \psi_{1}\right\rangle .
$$

Let $X=\mathbb{P}(V) \simeq \mathbb{C P}^{2}$. The relation (6.6) can be expressed as

$$
\psi_{2}=\Phi_{\mathrm{B}, \mathrm{C}}\left(\psi_{1}\right)
$$

where $\Phi_{\mathbf{B}, \mathbf{C}}: X \rightarrow X$ is the product of two "quadratic transformations"27

$$
\Phi_{\mathrm{B}, \mathrm{C}}=\Phi_{\mathrm{B}^{*}} \circ \Phi_{\mathrm{C}}
$$

with $\Phi_{\mathbf{C}}: X \rightarrow X^{*}$ the quadratic transformation taking the line $\langle\psi\rangle$ to the plane $\langle\psi, \mathbf{C} \psi\rangle$, and $\Phi_{\mathbf{B}^{*}}: X^{*} \rightarrow X$ the dual quadratic transformation taking a plane $p$ to the line $p \cap \mathbf{B} p$.

\footnotetext{
${ }^{27} \mathrm{~A}$ useful reference on quadratic transformations is [78].
} 
Thus $\Phi_{\mathbf{B}, \mathbf{C}}$ is a birational map (Cremona transformation) of degree 4, i.e. defined by three homogeneous degree 4 polynomials.

Thus $\psi_{2}$ is determined by $\psi_{1}$. Repeating this process using (6.3c), (6.3b) shows $\psi_{3}$ is determined by $\psi_{2}$, and $\psi_{1}$ is determined by $\psi_{3}$ :

$$
\psi_{3}=\Phi_{\mathbf{C}, \mathbf{A}}\left(\psi_{2}\right), \quad \psi_{1}=\Phi_{\mathbf{A}, \mathbf{B}}\left(\psi_{3}\right)
$$

Altogether, this means $\psi_{1}$ is constrained to obey

$$
\psi_{1}=\widehat{\Phi}\left(\psi_{1}\right)
$$

where $\widehat{\Phi}: X \rightarrow X$ is a degree 64 birational map

$$
\widehat{\Phi}=\Phi_{\mathrm{A}, \mathbf{B}} \circ \Phi_{\mathbf{C}, \mathbf{A}} \circ \Phi_{\mathbf{B}, \mathbf{C}} .
$$

Thus, whenever $\left(\psi_{1}, \psi_{2}, \psi_{3}\right)$ is a projective basis in special position, $\left\langle\psi_{1}\right\rangle \in X$ is a fixed point of $\widehat{\Phi}$, and (6.9) then determines the rest of the basis. This translates the job of finding projective bases in special position to the job of finding the fixed locus of $\widehat{\Phi}$.

This problem is simplified by the observation that $\widehat{\Phi}$ preserves the ratio of two cubic forms. Indeed, suppose we define a cubic form on $V$ by

$$
F_{M, M^{\prime}}(\psi)=\left[\psi, M \psi, M^{\prime} \psi\right],
$$

and dually on $V^{*}$

$$
F_{M, M^{\prime}}^{*}(\eta)=\left[\eta, M^{T} \eta, M^{\prime T} \eta\right]
$$

Then we have an identity of sextic forms on $V,^{28}$

$$
F_{M, M^{\prime}}^{*}\left(\Phi_{M^{\prime}}(\psi)\right)=F_{M, M^{\prime}}(\psi) F_{M^{\prime-1}, M}(\psi) .
$$

Now we consider the ratio of cubic forms

$$
r(\psi)=\frac{F_{\mathbf{C}, \mathbf{A}^{-1}}(\psi)}{F_{\mathbf{C}^{-1}, \mathbf{A}}(\psi)} .
$$

Using (6.14) six times we obtain the desired invariance:

$$
r(\widehat{\Phi}(\psi))=r(\psi) .
$$

(6.16) is equivalent to saying that $\widehat{\Phi}$ preserves a one-parameter family (pencil) of cubic curves $E_{t} \subset X$,

$$
E_{t}=\left\{F_{\mathbf{C}, \mathbf{A}^{-1}}(\psi)+t F_{\mathbf{C}^{-1}, \mathbf{A}}(\psi)=0\right\} \subset X .
$$

\footnotetext{
${ }^{28}$ We have no great insight into why this identity is true, although we have checked it in Mathematica; it is a specialization of a "remarkable identity" originally due to Zagier, given as equation 14 in [79].
} 
There are three points of $X$ which are common to all of the $E_{t}$, or said otherwise, this pencil of cubic curves has a base locus supported at three points of $X$. Two of the base points are easy to spot: if $\psi=e_{\mathbf{A}}$ or $\psi=e_{\mathbf{C}}$ then $F_{\mathbf{C}_{,} \mathbf{A}^{-1}}(\psi)=F_{\mathbf{C}^{-1}, \mathbf{A}}(\psi)=0$ and so $\psi$ lies on every $E_{t}$. The last base point is trickier: it is $p_{\mathbf{B}} \cap \mathbf{C}^{-1} p_{\mathbf{B}}$ where $p_{\mathbf{B}}$ is the unique plane fixed by $\mathbf{B}$. (Indeed if $\psi \in p_{\mathbf{B}} \cap \mathbf{C}^{-1} p_{\mathbf{B}}$ then $\psi, \mathbf{C} \psi$ and $\mathbf{A}^{-1} \psi$ all lie in $p_{\mathbf{B}}$, showing that $F_{\mathbf{C}, \mathbf{A}^{-1}}(\psi)=0$; similarly $F_{\mathbf{C}^{-1}, \mathbf{A}}(\psi)=0$.) These three base points lie on a line $\ell \subset X$. In fact the line $\ell$ (with multiplicity 3 ) is equal to $E_{t}$ for some $t=t_{*}$. Any point of $\ell$ is a fixed point of $\widehat{\Phi}$ (with the exception of the three base points, where $\widehat{\Phi}$ is not defined). This gives a 1-parameter family of projective bases in special position.

Now we want to see if there are any other fixed points. For this purpose the fact that $\widehat{\Phi}$ is not defined everywhere is technically inconvenient. To resolve its indeterminacies we blow up the base locus. This results in a singular surface, but by further blowing up the singular points, we obtain a smooth rational elliptic surface $\widetilde{X}$. See Figure 12.

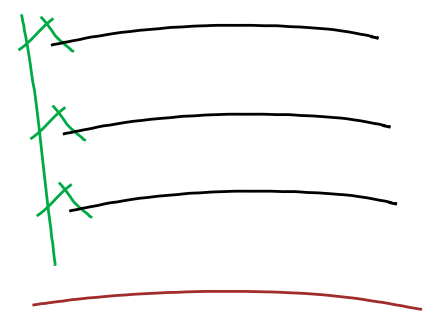

Figure 12: A neighborhood of the $I V^{*}$ fiber (green) in the rational elliptic surface $\widetilde{X}$. The preimage of each point of the base locus is a chain of three rational curves; two of the three are in the $I V^{*}$ fiber (green), while the last is a section of the elliptic fibration (black). There are generically 4 other singular fibers elsewhere in $\widetilde{X}$ (not shown).

$\widetilde{X}$ has one fiber of Kodaira type $I V^{*}$ (affine $E_{6}$ configuration), which maps to the line $E_{t_{*}}$ through the base points in $X$. This fiber has Euler characteristic 8. A smooth rational elliptic surface has Euler characteristic 12, and the smooth fibers do not contribute to the Euler characteristic, so there must be some other singular fibers in $\widetilde{X}$; the most generic possibility is to have 4 more singular fibers, each of type $I_{1}$ (nodal torus), so that altogether $\widetilde{X}$ has singular fibers $I V^{*}+4 I_{1}$. For some special A, B, C it may happen that some of the $I_{1}$ fibers collide. In particular, in Subsection 6.8 below we will meet a phenomenon where two $I_{1}$ fibers collide to make an $I I$ fiber (cusp), so that $\widetilde{X}$ has singular fibers $I V^{*}+I I+2 I_{1}$. The birational automorphism $\widehat{\Phi}$ of $X$ lifts to a regular map $\widetilde{X} \rightarrow X$, so in particular $\widehat{\Phi}$ acts by an honest automorphism of each fiber except for the $I V^{*}$ fiber. Since these fibers are (smooth or nodal) elliptic curves, their automorphism groups are easy to understand, and indeed by direct computations near a base point one can show that $\widehat{\Phi}$ is not trivial and not an inversion; thus it must act by a nontrivial translation on each fiber. It follows that the only place a fixed point can occur is at a singularity of a fiber; in particular, in the generic $I V^{*}+4 I_{1}$ case, the fixed points are exactly the 4 nodes. Combining these with the continuous family we found before, we conclude finally that $\widehat{\Phi}$ has " $4+\infty$ " fixed points, 
and thus there are " $4+\infty$ " projective bases in special position, as we claimed above.

This description of the projective bases in special position gives some small insight into their nature and their number, but more importantly for us, it is efficient enough to be used for numerical computations: starting from A, B, C, we use Mathematica to solve the polynomial system determining the singularities of the cubic curves (6.17); ${ }^{29}$ these give the desired basis elements $\psi_{1}$; then we determine $\psi_{2}$ and $\psi_{3}$ using (6.6), (6.9).

Finally let us comment on the case of A, B, C semisimple instead of unipotent. (This case would arise if, instead of the conformally invariant Minahan-Nemeschansky theory, we considered its mass deformation.) In this case the analysis is very similar to the above, except that the rational elliptic surface $\widetilde{X}$ which appears is a bit different: it arises by blowing up 9 distinct points of $X$ (lying on a cubic curve), instead of 3 with multiplicity. The result is that instead of singular fibers of type $I V^{*}+4 I_{1}$ one generically gets $12 I_{1}$, and so instead of " $4+\infty$ " $\mathcal{W}$-abelianizations there are generically just $12 \mathcal{W}$-abelianizations.

\subsection{The spectral coordinates}

Now we are in a position to decribe the spectral coordinates concretely.
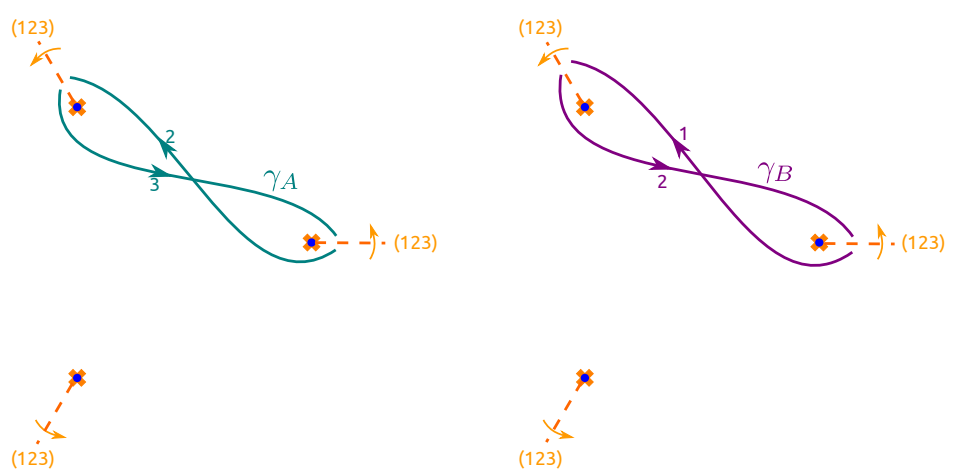

Figure 13: Cycles $\gamma_{A}$ and $\gamma_{B}$ on the 3 -fold branched cover $\Sigma$.

Let $\gamma_{A}, \gamma_{B}$ be the cycles on $\Sigma$ shown in Figure 13. Fix an SL(3)-connection $\nabla$ over $C$, with unipotent holonomy, and fix a $\mathcal{W}$-abelianization of $\nabla$. Let $\left(\psi_{1}, \psi_{2}, \psi_{3}\right)$ be the corresponding basis of solutions near $z=0$. As we have explained above, $\left(\psi_{1}, \psi_{2}, \psi_{3}\right)$ are in special position. Then, the spectral coordinates are (see Appendix A)

$$
\begin{aligned}
\mathcal{X}_{A} & =\frac{\left[\psi_{2}, \psi_{3}, \psi_{1}\right]}{\left[\mathbf{C}^{-1} \psi_{3}, \mathbf{A} \psi_{2}, \psi_{1}\right]} \\
\mathcal{X}_{B} & =\sqrt{-\frac{\left[\mathbf{C} \psi_{1}, \mathbf{B}^{-1} \psi_{2}, \psi_{3}\right]\left[\mathbf{C} \psi_{1}, \psi_{1}, \psi_{3}\right]\left[\psi_{2}, \mathbf{A} \psi_{2}, \psi_{1}\right]\left[\mathbf{B} \psi_{3}, \mathbf{A}^{-1} \psi_{1}, \psi_{2}\right]\left[\mathbf{B} \psi_{3}, \psi_{3}, \psi_{2}\right]}{\left[\psi_{2}, \mathbf{B}^{-1} \psi_{2}, \psi_{3}\right]\left[\mathbf{C}^{-1} \psi_{3}, \mathbf{A} \psi_{2}, \psi_{1}\right]\left[\mathbf{C}^{-1} \psi_{3}, \psi_{3}, \psi_{1}\right]\left[\psi_{1}, \psi_{3}, \psi_{2}\right]\left[\psi_{1}, \mathbf{A}^{-1} \psi_{1}, \psi_{2}\right]}} .
\end{aligned}
$$

\footnotetext{
${ }^{29}$ In particular this seems to be much more efficient than trying to solve the coplanarity constraints (6.3) directly.
} 


\subsection{Spectral coordinates for the continuous family of abelianizations}

In this section we record an interesting curiosity, not required for the rest of the paper.

Recall that there is a continuous family of $\mathcal{W}$-abelianizations, with the property that $\psi_{1}$ is a linear combination of the eigenvectors of $\mathbf{A}$ and $\mathbf{C}$, and similarly for $\psi_{2}, \psi_{3}$. It turns out that the spectral coordinates $\mathcal{X}_{A}$ and $\mathcal{X}_{B}$ are independent of which member of the continuous family we take, so all of these $\mathcal{W}$-abelianizations are actually isomorphic, and in some sense they should be considered as just one abelianization. Moreover, these spectral coordinates are Fock-Goncharov coordinates associated to an ideal triangulation of $\mathbb{C P}^{1} .{ }^{30}$ Indeed, let $a_{1}$ be an eigenvector for $\mathbf{A}$, and $a_{2}$ another vector such that $\left\langle a_{1}, a_{2}\right\rangle$ is the unique plane preserved by $\mathbf{A}$; likewise define $b_{1}, b_{2}$ and $c_{1}, c_{2}$, and $d_{1}, d_{2}$ associated to the operator $\mathbf{D}=\mathbf{C}^{-1} \mathbf{B C}$. Then the triple ratio and edge coordinate from [21] are

$$
t=\frac{\left[a_{1}, a_{2}, b_{1}\right]\left[b_{1}, b_{2}, c_{1}\right]\left[c_{1}, c_{2}, a_{1}\right]}{\left[a_{1}, a_{2}, c_{1}\right]\left[b_{1}, b_{2}, a_{1}\right]\left[c_{1}, c_{2}, b_{1}\right]}, \quad e=\frac{\left[b_{1}, c_{1}, a_{1}\right]\left[d_{1}, a_{2}, a_{1}\right]}{\left[a_{2}, c_{1}, a_{1}\right]\left[b_{1}, d_{1}, a_{1}\right]} .
$$

These coordinates turn out to be related to the spectral coordinates for the continuous family of $\mathcal{W}$-abelianizations, by

$$
\mathcal{X}_{A}=e, \quad \mathcal{X}_{B}=\frac{t}{\sqrt{e}} .
$$

It is not clear to us why the Fock-Goncharov coordinates appear as spectral coordinates for the Stokes graph $\mathcal{W}$. In [27] it was shown that Fock-Goncharov coordinates do appear as spectral coordinates for a specific sort of spectral network associated to a triangulation, but that is a different spectral network from $\mathcal{W}$. It would be interesting to understand this better.

At any rate, the Fock-Goncharov coordinates will not play much role in the rest of the paper; most of our attention will be focused instead on the 4 discrete abelianizations, since these are the ones which turn out to be directly related to WKB for the $T_{3}$ equation.

\subsection{The monodromy matrices}

Relative to the projective basis $\left(\psi_{1}, \psi_{2}, \psi_{3}\right)$ we can write the monodromy explicitly. Its form depends on which $\mathcal{W}$-abelianization we take. For the 4 discrete $\mathcal{W}$-abelianizations, it

\footnotetext{
${ }^{30}$ The triangulation is made up of 2 triangles, whose interiors are $\{|z|<1\}$ and $\{|z|>1\}$.
} 
is (up to a diagonal gauge transformation)

$$
\begin{aligned}
& \mathbf{A}=\left(\begin{array}{ccc}
-f\left(\mathcal{X}_{A}\right) \mathcal{X}_{A} & 0 & \mathcal{X}_{A} \mathcal{X}_{B}^{-1} \sqrt{1+f\left(\mathcal{X}_{A}\right)^{2} \mathcal{X}_{A}} \\
\left(1+f\left(\mathcal{X}_{A}\right)^{2} \mathcal{X}_{A}\right) \mathcal{X}_{B} & f\left(\mathcal{X}_{A}\right) & -f\left(\mathcal{X}_{A}\right) \mathcal{X}_{A} \sqrt{1+f\left(\mathcal{X}_{A}\right)^{2} \mathcal{X}_{A}} \\
f\left(\mathcal{X}_{A}\right) \mathcal{X}_{B} \sqrt{1+f\left(\mathcal{X}_{A}\right)^{2} \mathcal{X}_{A}} & \mathcal{X}_{A}^{-1} \sqrt{1+f\left(\mathcal{X}_{A}\right)^{2} \mathcal{X}_{A}} & -f\left(\mathcal{X}_{A}\right)^{2} \mathcal{X}_{A}
\end{array}\right), \\
& \mathbf{B}=\left(\begin{array}{ccc}
-f\left(\mathcal{X}_{A}\right)^{2} \mathcal{X}_{A} & f\left(\mathcal{X}_{A}\right) \sqrt{1+f\left(\mathcal{X}_{A}\right)^{2} \mathcal{X}_{A}} & \mathcal{X}_{B}^{-1} \sqrt{1+f\left(\mathcal{X}_{A}\right)^{2} \mathcal{X}_{A}} \\
\mathcal{X}_{A} \sqrt{1+f\left(\mathcal{X}_{A}\right)^{2} \mathcal{X}_{A}} & -f\left(\mathcal{X}_{A}\right) \mathcal{X}_{A} & 0 \\
-f\left(\mathcal{X}_{A}\right) \mathcal{X}_{B} \sqrt{1+f\left(\mathcal{X}_{A}\right)^{2} \mathcal{X}_{A}} & \left(1+f\left(\mathcal{X}_{A}\right)^{2} \mathcal{X}_{A}\right) \mathcal{X}_{B} \mathcal{X}_{A}^{-1} & f\left(\mathcal{X}_{A}\right)
\end{array}\right), \\
& \mathbf{C}=\left(\begin{array}{ccc}
f\left(\mathcal{X}_{A}\right) & -f\left(\mathcal{X}_{A}\right) \sqrt{1+f\left(\mathcal{X}_{A}\right)^{2} \mathcal{X}_{A}} & 1+f\left(\mathcal{X}_{A}\right)^{2} \mathcal{X}_{A} \\
\sqrt{1+f\left(\mathcal{X}_{A}\right)^{2} \mathcal{X}_{A}} & -f\left(\mathcal{X}_{A}\right)^{2} \mathcal{X}_{A} & f\left(\mathcal{X}_{A}\right) \mathcal{X}_{A} \sqrt{1+f\left(\mathcal{X}_{A}\right)^{2} \mathcal{X}_{A}} \\
0 & \sqrt{1+f\left(\mathcal{X}_{A}\right)^{2} \mathcal{X}_{A}} & -f\left(\mathcal{X}_{A}\right) \mathcal{X}_{A}
\end{array}\right),
\end{aligned}
$$

where

$$
f\left(\mathcal{X}_{A}\right)=\frac{1-\mathcal{X}_{A} \pm \sqrt{1-14 \mathcal{X}_{A}+\mathcal{X}_{A}^{2}}}{2 \mathcal{X}_{A}} .
$$

The formulas (6.21) can be obtained directly by "nonabelianization:" we begin with $\nabla^{\mathrm{ab}}$ and reconstruct $\nabla$ from it, using only the constraint that the gluing matrices across Stokes curves are of the block form (5.6).

It we choose the - sign in (6.21d), then $f\left(\mathcal{X}_{A}\right)$ is regular at $\mathcal{X}_{A}=0$, with an expansion of the form

$$
f\left(\mathcal{X}_{A}\right)=3 \mathcal{X}_{A}+12 \mathcal{X}_{A}^{2}+\cdots
$$

This expansion played an important role in the analysis of BPS particles of the MinahanNemeschansky $E_{6}$ theory in [34]; its coefficients count BPS solitons in the MinahanNemeschansky theory coupled to a certain $\frac{1}{2}$-BPS surface defect.

The - branch of the square root is also the one which appears for the $\mathcal{W}$-abelianization coming from exact WKB: when we take $u>0$ and $\hbar \rightarrow 0$ with $\arg \hbar=0$, the WKB abelianization has $\mathcal{X}_{A}$ exponentially small, and likewise $f\left(\mathcal{X}_{A}\right)$ exponentially small. On the other hand, when $\mathcal{X}_{A}$ is not small, there is in general no canonical choice of branch in (6.21d); both possibilities are possible. This suggests that we should pay attention to the locus where the branches collide: this occurs when $1-14 \mathcal{X}_{A}+\mathcal{X}_{A}^{2}=0$ ie $\mathcal{X}_{A}=7 \pm 4 \sqrt{3}$. Indeed this locus will turn out to be important below.

\subsection{Testing the predictions of WKB}

As we have described, when $u>0$ and $\hbar>0$, we conjecture that the higher-rank exact WKB method with $\vartheta=0$ furnishes a $\mathcal{W}$-abelianization of the SL(3)-connection associated to the $T_{3}$ equation. 
In fact we can go a bit further: since the $T_{3}$ equation depends only on $u^{\prime}=u / \hbar^{3}$, we could equally well study it by using exact WKB with $u>0$ but $\vartheta=\arg \hbar=\frac{2 \pi}{3}$, or $\vartheta=\arg \hbar=\frac{4 \pi}{3}$. The corresponding Stokes graphs $\mathcal{W}^{\vartheta}$ are not equal to $\mathcal{W}=\mathcal{W}^{\vartheta=0}$, but differ from $\mathcal{W}$ only by cyclic permutations of the sheet labels (123). Thus the $\mathcal{W}^{\vartheta}$ abelianization provided by exact $\mathrm{WKB}$ can be converted to a $\mathcal{W}$-abelianization, by cyclically permuting the projective basis $\left(\psi_{1}, \psi_{2}, \psi_{3}\right)$. In this way exact WKB should produce two additional $\mathcal{W}$-abelianizations $A_{\vartheta}$.

Altogether then, we expect that for $u^{\prime} \gg 0$, among the $\mathcal{W}$-abelianizations of the $T_{3}$ equation we should find three coming from exact $\left.\mathrm{WKB}, A_{\vartheta} \vartheta=0, \frac{2 \pi}{3}, \frac{4 \pi}{3}\right)$. The spectral coordinates associated to these three $\mathcal{W}$-abelianizations should have the small- $\hbar$ asymptotic behavior

$$
\mathcal{X}_{\gamma} \approx \exp \left(Z_{\gamma}(u) / \hbar\right), \quad \arg \hbar=\vartheta .
$$

In fact, these asymptotics should hold not only for $\arg \hbar=\vartheta$ but more generally for $\arg \hbar \in\left(\vartheta-\frac{\pi}{2}, \vartheta+\frac{\pi}{2}\right)$.

It is convenient to rewrite these asymptotics in terms of the invariant parameter $u^{\prime}$, using the explicit formulas for the periods:

$$
Z_{A}=-M u^{\frac{1}{3}}, \quad Z_{B}=-\mathrm{e}^{-\frac{2 \pi \mathrm{i}}{3}} M u^{\frac{1}{3}},
$$

where

$$
M=2^{-\frac{2}{3}} \pi^{-\frac{1}{2}} \Gamma\left(\frac{1}{3}\right) \Gamma\left(\frac{1}{6}\right) \approx 5.2999 .
$$

Then the prediction is

$$
\mathcal{X}_{A} \approx \exp \left(-M \mathrm{e}^{-\mathrm{i} \vartheta} u^{\prime \frac{1}{3}}\right), \quad \mathcal{X}_{B} \approx \exp \left(-M \mathrm{e}^{-\mathrm{i}\left(\vartheta+\frac{2 \pi}{3}\right)} \mathcal{u}^{\prime \frac{1}{3}}\right),
$$

This should hold for $u^{\prime} \gg 0$ but also more generally when $u^{\prime}$ is analytically continued; in fact, since changing $\arg \hbar$ by $\frac{\pi}{2}$ changes $\arg u^{\prime}$ by $\frac{3 \pi}{2}$, the prediction (6.26) can be analytically continued to give the asymptotics as $u^{\prime} \rightarrow \infty$ along an arbitrary ray.

We can test this prediction experimentally as follows:

- Numerically compute the monodromy matrices A, B, C for the $T_{3}$ equation, for various values of $u^{\prime}$.

- Use the method of Subsection 6.3 to determine the $\mathcal{W}$-abelianizations for each $u^{\prime}$.

- Use the formulas (6.18a), (6.18b) to compute the spectral coordinates $\mathcal{X}_{A}$ and $\mathcal{X}_{B}$ for each abelianization.

- Check that 3 of the $\mathcal{W}$-abelianizations have the behavior (6.26) when $\left|u^{\prime}\right| \rightarrow \infty$.

Experimentally this indeed works; for a sample of the numerical evidence, see Figure 14. 

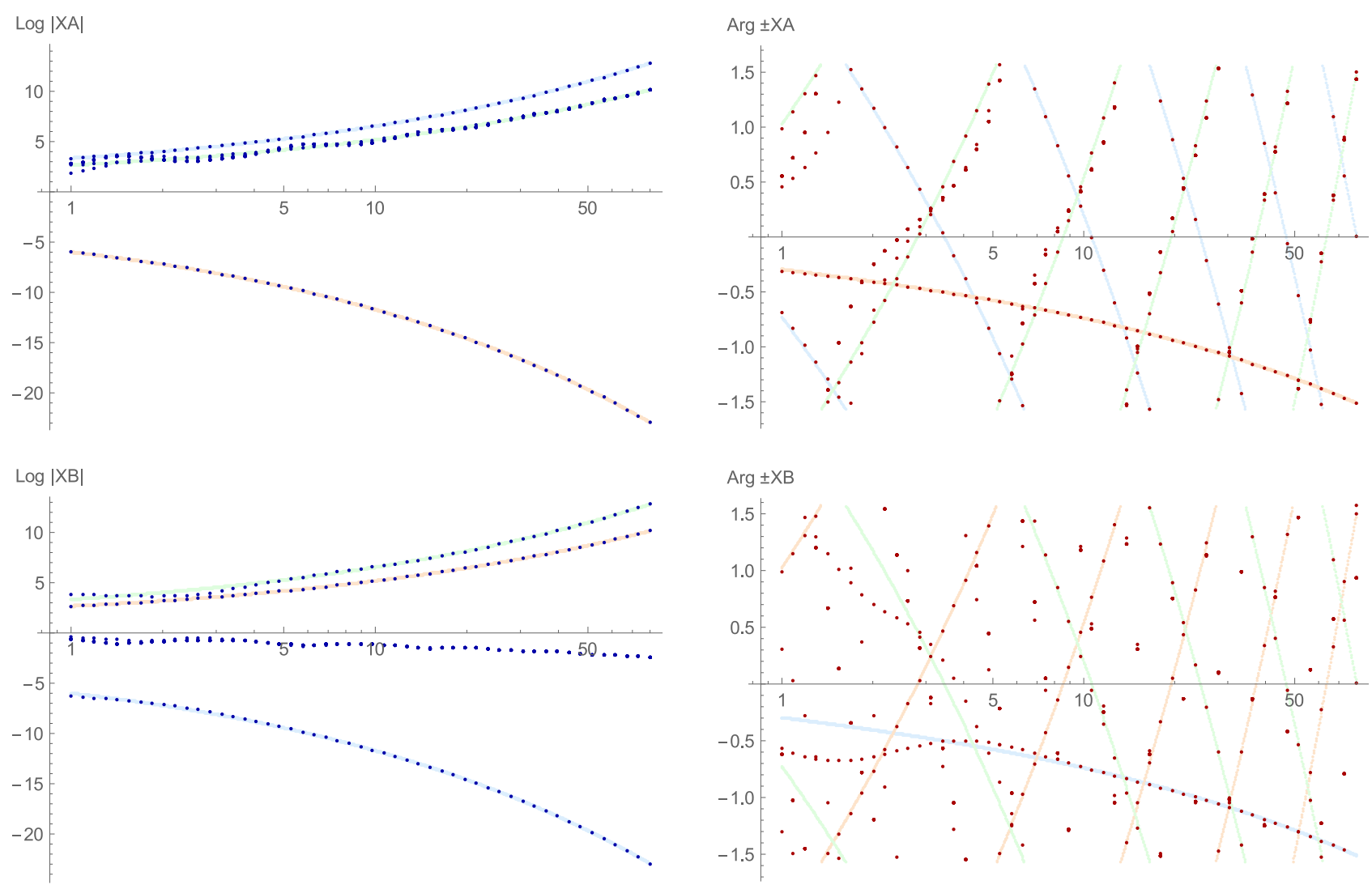

Figure 14: A numerical study of $\mathcal{X}_{A}\left(u^{\prime}\right)$ and $\mathcal{X}_{B}\left(u^{\prime}\right)$ for $\arg u^{\prime}=0.2$ and $1<\left|u^{\prime}\right|<80$. For each value of $u^{\prime}$, the values of $\mathcal{X}_{A}$ and $\mathcal{X}_{B}$ for all of the $\mathcal{W}$-abelianizations are plotted. The $3 \mathrm{WKB}$ asymptotic formulas are also plotted, with $\vartheta=0$ (orange), $\vartheta=-\frac{2 \pi}{3}$ (blue), $\vartheta=\frac{2 \pi}{3}$ (green). In each case the curve plotted is the sum of the first three terms of the WKB asymptotic series.

Finally we consider what happens for $-u^{\prime} \gg 0$. We can reach this situation by taking $u>0$ and $\hbar<0$. The resulting Stokes graph $\mathcal{W}^{\vartheta=\pi}$ is identical to $\mathcal{W}$, except that the sheet labels are reversed. Because all walls of $\mathcal{W}$ are double, the notion of $\mathcal{W}$-abelianization is actually unaffected by this reversal of the sheet labels; a $\mathcal{W}^{\vartheta=\pi}$-abelianization is the same thing as a $\mathcal{W}$-abelianization. Then, in parallel to $u^{\prime} \gg 0$, exact WKB at the three phases $\vartheta=\arg \hbar=\pi, \frac{5 \pi}{3}, \frac{\pi}{3}$ gives three $\mathcal{W}$-abelianizations $A_{\vartheta}$ of the $T_{3}$ equation with $-u^{\prime} \gg 0$.

\subsection{Analytic continuation}

Now let us consider the analytic continuation of the spectral coordinates $\mathcal{X}_{\gamma}$ in $u$ and $\hbar$. The $\mathcal{X}_{\gamma}$ are really defined on the 4 -fold cover given by the discrete $\mathcal{W}$-abelianizations; thus studying their monodromy is equivalent to studying the monodromy of the $\mathcal{W}$ abelianizations. Since the $T_{3}$ equation depends only on $u^{\prime}=u / \hbar^{3}$ this reduces to working out the monodromy in the $u^{\prime}$-plane. We have not found an analytic way of computing this monodromy, but we have studied it numerically, by tracking the spectral coordinates $\mathcal{X}_{A}$ 
and $\mathcal{X}_{B}$ directly as functions of $u^{\prime}$.

Let us begin with large $\left|u^{\prime}\right|$. As we have discussed above, at either $u^{\prime} \gg 0$ or $u^{\prime} \ll 0$ we have three $\mathcal{W}$-abelianizations $A_{\vartheta}$ coming from WKB. As we continue counterclockwise from one side to the other, these three $\mathcal{W}$-abelianizations continue as $A_{\vartheta} \rightarrow A_{\vartheta+\frac{\pi}{3}}$; thus, going counterclockwise around a large circle in the $u^{\prime}$-plane induces the order-3 monodromy $A_{\vartheta} \rightarrow A_{\vartheta+\frac{2 \pi}{3}}$. The behavior of $\mathcal{X}_{A}$ as we go around the circle $\left|u^{\prime}\right|=25$ is shown in Figure 15.

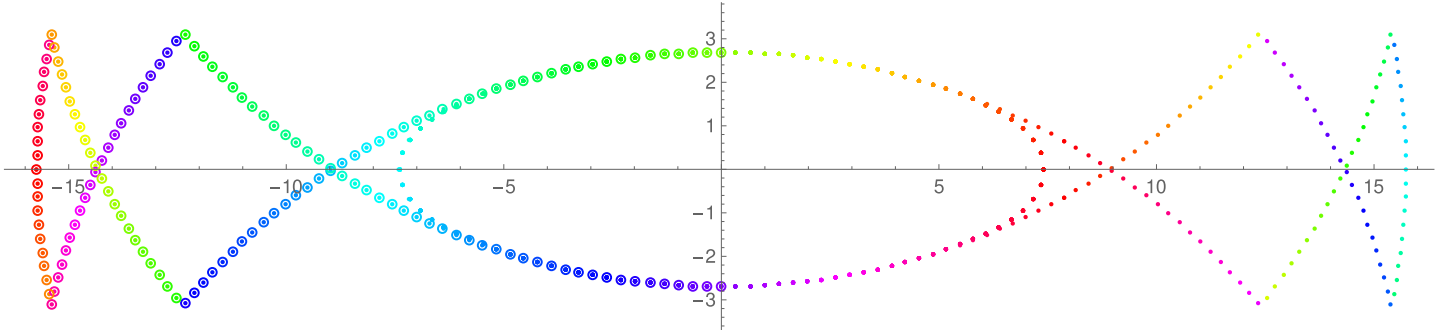

Figure 15: The coordinate $\log \mathcal{X}_{A}\left(u^{\prime}\right)$, plotted in $\mathbb{C} / 2 \pi \mathrm{i} \mathbb{Z}$, for $\left|u^{\prime}\right|=25$. The hue indicates the phase $\arg u^{\prime}$. For each value of $u^{\prime}$, there are 4 solid points on the plot, representing the values of $\mathcal{X}_{A}\left(u^{\prime}\right)$ for the 4 discrete $\mathcal{W}$-abelianizations. 3 of these points lie on a large loop, while the fourth point lies on a smaller loop; the two loops come very close to one another. As arg $u^{\prime}$ advances by $2 \pi, \mathcal{X}_{A}\left(u^{\prime}\right)$ moves one-third of the way around the large loop, or all the way around the small loop. This reflects the fact that the monodromy permutes 3 of the discrete $\mathcal{W}$-abelianizations while leaving the fourth one invariant. The hollow circles on the plot show the WKB asymptotic formula for $\mathcal{X}_{A}\left(u^{\prime}\right)$, analytically continued from $\arg u^{\prime}=0$ to the region $-\frac{3 \pi}{2}<\arg u^{\prime}<\frac{3 \pi}{2}$; the fact that these points track closely with one of the 4 $\mathcal{W}$-abelianizations in this range confirms the prediction of WKB.

Now we can ask what happens in the interior of the $u^{\prime}$-plane. By numerical exploration we found monodromy around just two points, located at $u^{\prime}= \pm u_{*}^{\prime}$, where $u_{*}^{\prime} \approx 0.041992794$. Coming in from $u^{\prime} \gg 0$, we find that the two $\mathcal{W}$-abelianizations which we called $A_{\frac{2 \pi}{3}}$ and $A_{\frac{4 \pi}{3}}$ above collide at $u^{\prime}=u_{*}^{\prime}$. When they collide they have $\mathcal{X}_{A}=7+4 \sqrt{3}$ and $\left|\mathcal{X}_{B}\right|^{-2}=\mathcal{X}_{A}$. Traveling around a small loop around $u_{*}^{\prime}$, these two $\mathcal{W}$-abelianizations are exchanged. Similarly, coming in from $u^{\prime} \ll 0$, we find that the two $\mathcal{W}$-abelianizations we called $A_{\frac{\pi}{3}}$ and $A_{\frac{5 \pi}{3}}$ are exchanged around $u^{\prime}=-u_{*}^{\prime}$, with $\mathcal{X}_{A}=7-4 \sqrt{3}$ there.

By numerical experimentation we have not found monodromy anywhere else in the $u^{\prime}$ plane. Thus we conjecture that the only monodromy is around $\pm u_{*}^{\prime}$. It is straightforward to check that this gives a consistent global picture: the order-3 monodromy we found at large $\left|u^{\prime}\right|$ can be factorized into the two order- 2 monodromies around $\pm u_{*}^{\prime}$.

It is interesting to compare the monodromy of the $\mathcal{X}_{\gamma}$ with that of the periods $Z_{\gamma}$. At large $\left|u^{\prime}\right|$ the two monodromies agree. At small $\left|u^{\prime}\right|$ the $Z_{\gamma}$ have a single singularity at $u^{\prime}=0$, while the $\mathcal{X}_{\gamma}$ have two singularities at $\pm u_{*}^{\prime}$. Since $\mathcal{X}_{\gamma} \sim \exp \left(Z_{\gamma} / \hbar\right)$ one might wonder whether one can globally take logs, to obtain a deformation $\widetilde{Z}_{\gamma}=\hbar \log \mathcal{X}_{\gamma}(\hbar)$. 
Were this possible, we would just have two holomorphic functions $\widetilde{Z}_{\gamma}$ in the $u^{\prime}$-plane, transforming linearly under monodromy around the two points $\pm u_{*}^{\prime}$. Then it would be tempting to try to realize the $\widetilde{Z}_{\gamma}$ directly as periods of a globally defined 1-form on a family of deformed spectral curves. The real situation is more delicate, because the analytically continued functions $\mathcal{X}_{\gamma}$ may have zeroes or poles at some values of $u^{\prime}$; upon analytic continuation around such a $u^{\prime}, \widetilde{Z}_{\gamma}$ has an additive shift by $\pm 2 \pi \mathrm{i} \hbar$. To see examples of this kind of singularity concretely, we plot the spectral coordinates for all abelianizations on the line $u^{\prime}>0$ : see Figure 16 .

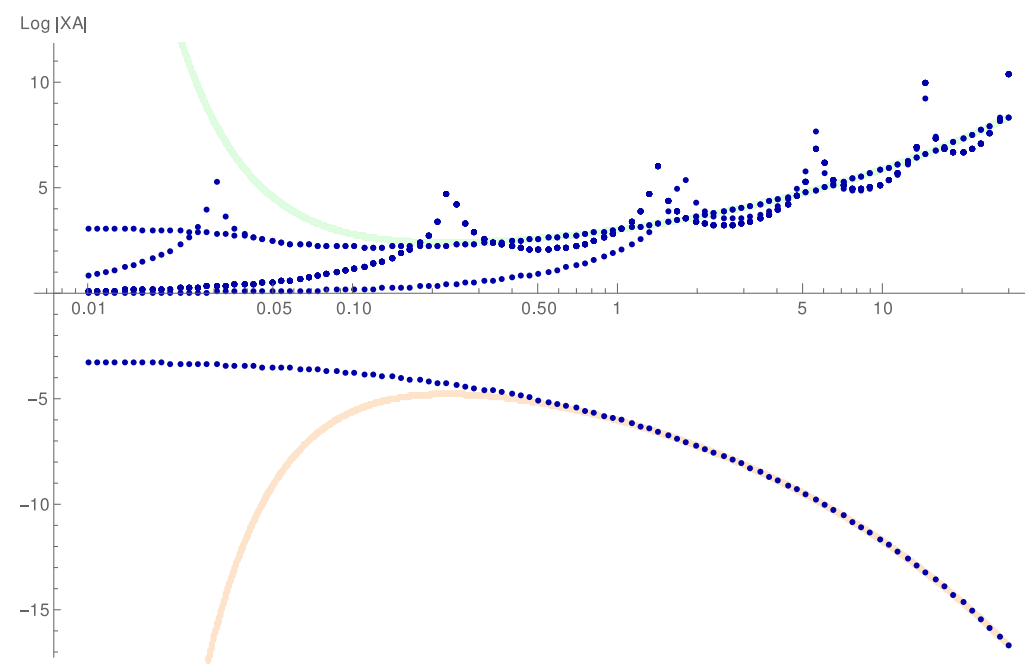

Figure 16: A numerical study of the spectral coordinate $\mathcal{X}_{A}\left(u^{\prime}\right)$ for $0.01<u^{\prime}<30$. Notation is as in Figure 14.

Along the ray $u^{\prime}>0$ there appear to be infinitely many such singularities, with the first few at $u^{\prime} \approx 0.03013837,0.23370955,1.75819973, \ldots$. Similarly along the ray $u^{\prime} \in \mathrm{i} \mathbb{R}_{+}$there are singularities which occur at $u^{\prime} \approx 0.4595 \mathrm{i}, \ldots$

So far we have been discussing the $\mathcal{W}$-abelianizations which occur discretely. For the $\mathcal{W}$-abelianization which occurs in a continuous family, the situation is simpler: there is no monodromy mixing it with the other $\mathcal{W}$-abelianizations. This matches with the fact from Subsection 6.5 that the corresponding spectral coordinates are the Fock-Goncharov coordinates, which are uniquely determined by the connection $\nabla$ as long as each of $\mathbf{A}, \mathbf{B}$, $\mathrm{C}$ preserves a unique flag.

\subsection{The uniformization point}

It is also interesting to ask what happens at $u^{\prime}=0$. This point is a singularity for the periods $Z_{\gamma}$, but the $T_{3}$ equation at $u^{\prime}=0$ is perfectly regular. Indeed, its monodromy representation can be described explicitly, because it has a simple interpretation: it is the image of the uniformization representation of the 3-punctured sphere, $\pi_{1}(C) \rightarrow$ $\Gamma_{0}(2) \subset \mathrm{SL}(2, \mathbb{Z})$, under the symmetric square $\operatorname{Sym}^{2}: \operatorname{SL}(2, \mathbb{Z}) \rightarrow \operatorname{SL}(3, \mathbb{Z})$. Thus it can 
be represented explicitly by the matrices

$$
\begin{aligned}
& \mathbf{A}=\operatorname{Sym}^{2}\left(\begin{array}{ll}
1 & 2 \\
0 & 1
\end{array}\right)=\left(\begin{array}{lll}
1 & 2 & 4 \\
0 & 1 & 4 \\
0 & 0 & 1
\end{array}\right), \\
& \mathbf{B}=\operatorname{Sym}^{2}\left(\begin{array}{cc}
1 & 0 \\
-2 & 1
\end{array}\right)=\left(\begin{array}{ccc}
1 & 0 & 0 \\
-4 & 1 & 0 \\
4 & -2 & 1
\end{array}\right), \\
& \mathbf{C}=(\mathbf{A B})^{-1}=\left(\begin{array}{lll}
1 & -2 & 4 \\
4 & -7 & 12 \\
4 & -6 & 9
\end{array}\right) .
\end{aligned}
$$

The integrality of these matrices implies that the spectral coordinates $\mathcal{X}_{A}, \mathcal{X}_{B}$ are algebraic. Indeed, we have computed them explicitly: at $u^{\prime}=0$ two of the discrete $\mathcal{W}$-abelianizations have

$$
\left(\mathcal{X}_{A}, \mathcal{X}_{B}\right)=\left(\frac{1}{5}(59 \pm 24 \sqrt{6}), \sqrt{\frac{1}{5}(59 \mp 24 \sqrt{6})}\right)
$$

and the other two have the coincident value

$$
\left(\mathcal{X}_{A}, \mathcal{X}_{B}\right)=(-1,1)
$$

while the continuous family of $\mathcal{W}$-abelianizations have

$$
\left(\mathcal{X}_{A}, \mathcal{X}_{B}\right)=(1,1) \text {. }
$$

If we approach $u^{\prime}=0$ starting from $u^{\prime} \gg 0$, the WKB abelianization $A_{0}$ smoothly approaches the one with $\mathcal{X}_{A}=\frac{1}{5}(59-24 \sqrt{6}) \approx 0.0424492$ (see the bottom curve in Figure 16).

\subsection{Integral equations}

Now let us consider the construction of an integral equation (2.19) obeyed by the spectral coordinates, following the scheme of Subsection 2.6. For concreteness, we fix $u>0$ (it is easy to restore more general $u$ dependence if needed.)

In the scheme of Subsection 2.6 we have to choose a function $\vartheta(\arg \hbar)$. It would be inconvenient in this example to choose $\vartheta(\arg \hbar)=\arg \hbar$; the results of [34] imply that there are infinitely many active rays, and indeed the active rays are everywhere dense. We pick instead

$$
\vartheta(\arg \hbar)=n \frac{\pi}{3} \quad \text { for } \quad \arg \hbar \in\left(n \frac{\pi}{3}-\frac{\pi}{6}, n \frac{\pi}{3}+\frac{\pi}{6}\right) .
$$

This choice has the effect of collapsing the infinitely many active rays down to 6 rays $r_{n}$ with phases $\frac{\pi}{6}+n \frac{\pi}{3}$. To write the integral equation (2.19) we need to determine the functions $F_{r_{n}, \gamma}$ attached to those 6 rays. According to (2.21), this amounts to determining the coordinate transformation which relates the spectral coordinates $\mathcal{X}^{\vartheta=n \frac{\pi}{3}}$ to the $\mathcal{X}^{\vartheta=(n+1) \frac{\pi}{3}}$. 
To be concrete let us focus on the ray $r_{0}$, with phase $\frac{\pi}{6}$; the others are essentially the same. The functions $x_{\gamma}=\mathcal{X}_{\gamma}^{\vartheta=0}(\hbar)$ for $\arg \hbar=0$, and the functions $y_{\gamma}=\mathcal{X}_{\gamma}^{\vartheta=\frac{\pi}{3}}(\hbar)$ for $\arg \hbar=\frac{\pi}{3}$, are associated to a $\mathcal{W}$-abelianization and a $\mathcal{W}^{\vartheta=\frac{\pi}{3}}$-abelianization respectively. We analytically continue $x$ and $y$ to a common sector $\arg \hbar \in\left(-\epsilon, \frac{\pi}{3}+\epsilon\right)$, which in particular contains $r_{0}$. In this sector $x_{\gamma}$ and $y_{\gamma}$ have the same asymptotics as $\hbar \rightarrow 0$, but they are not the same; the "nonperturbative" difference between them, $F_{r_{0}, \gamma}=y_{\gamma} / x_{\gamma}$, is what we are after.

We can describe this difference a bit more concretely. Just as in Subsection 6.7, note that a $\mathcal{W}^{\vartheta=\frac{\pi}{3}}$-abelianization also induces a $\mathcal{W}$-abelianization. In fact, the $x_{\gamma}$ are the spectral coordinates for the $\mathcal{W}$-abelianization $A_{0}$, while the $y_{\gamma}$ are obtained by applying a cyclic permutation of the basis cycles, $(A, B,-A-B) \rightarrow(B,-A-B, A)$, to the spectral coordinates for the $\mathcal{W}$-abelianization $A_{-\frac{2 \pi}{3}}$. (For example, $x_{B}$ is given by the points along the orange curve in the lower part of Figure 14, while $y_{B}$ is given by the points along the green curve in the upper part of that figure.)

We do not have a closed formula for the coordinate transformation $y=\mathbf{S}_{0, \frac{\pi}{3}}(x)$ giving the $y_{\gamma}$ as a function of the $x_{\gamma}$. However, we do have some partial information. As we vary $\vartheta$ from 0 to $\frac{\pi}{3}$, the $\vartheta$-Stokes graph jumps at a countable dense set of phases, and correspondingly $\mathbf{S}_{0, \frac{\pi}{3}}$ admits a factorization into a countable product of Stokes automorphisms, of the form $[80,73,16]$

$$
\mathbf{S}_{0, \frac{\pi}{3}}=\mathbf{T}_{\frac{\pi}{3}}^{\frac{1}{2}} \circ\left(\bigcap_{\vartheta \in\left(0, \frac{\pi}{3}\right)}^{\curvearrowright} \mathbf{T}_{\vartheta}\right) \circ \mathbf{T}_{0}^{\frac{1}{2}} .
$$

In (6.34) the product over $\vartheta$ is taken in decreasing order, $\mathbf{T}_{\vartheta}$ is a coordinate transformation of the form

$$
\mathbf{T}_{\vartheta}=\prod_{\gamma: \arg \left(-Z_{\gamma}\right)=\vartheta} \mathcal{K}_{\gamma}^{\Omega(\gamma)}, \quad \mathcal{K}_{\gamma}^{*} \mathcal{X}_{\mu}=\mathcal{X}_{\mu}\left(1-\sigma(\gamma) \mathcal{X}_{\gamma}\right)^{\langle\mu, \gamma\rangle},
$$

$\sigma: H_{1}(\Sigma, \mathbb{Z}) \rightarrow\{ \pm 1\}$ is

$$
\sigma\left(a \gamma_{A}+b \gamma_{B}\right)=(-1)^{a+b+a b},
$$

and most crucially, there appear some integers $\Omega(\gamma) \in \mathbb{Z}$, determined by the jumping of the Stokes graphs. In the relation to $\mathcal{N}=2$ supersymmetric field theory, $\Omega(\gamma)$ is a helicity supertrace counting BPS particles with charge $\gamma$. Note that $\mathbf{T}_{\vartheta}=1$ except for countably many phases $\vartheta, 31$ and for each such phase $\mathbf{T}_{\vartheta}$ is a countable product, so altogether the product in (6.34) involves a countably infinite number of $\mathcal{K}_{\gamma}$.

The effect of the transformation $\mathcal{K}_{\gamma}$ is to multiply each $\mathcal{X}_{\mu}$ by some power of $\left(1 \pm \mathcal{X}_{\gamma}\right)$. For the $\mathcal{K}_{\gamma}$ which contribute to $\mathbf{S}_{0, \frac{\pi}{3}}$ we have $\arg \left(-Z_{\gamma}\right) \in\left[0, \frac{\pi}{3}\right]$. When $\arg \hbar \in\left(-\epsilon, \frac{\pi}{3}+\epsilon\right)$, these $\mathcal{X}_{\gamma}$ are exponentially suppressed like $\exp \left(Z_{\gamma} / \hbar\right)$ as $\hbar \rightarrow 0$, and thus $\mathcal{K}_{\gamma}$ acts by an exponentially small transformation on the coordinates. In particular, if $\arg \hbar=\frac{\pi}{6}$, then for

\footnotetext{
${ }^{31}$ The results of [34] show that $\Omega(\gamma) \neq 0$ for every primitive charge $\gamma$, so all of the countably many phases $\vartheta$ which could give nontrivial $\mathbf{T}_{\vartheta}$ indeed do.
} 
$\gamma=(a, b)=a \gamma_{A}+b \gamma_{B}, \operatorname{Re}\left(-Z_{\gamma} / \hbar\right)$ is proportional to $a-b$; all $(a, b)$ which contribute have $a-b>0$, and of those the least suppressed $\mathcal{K}_{a, b}$ are the ones with $a-b=1$, next are the ones with $a-b=2$, and so on.

We do not know all of the $\Omega(\gamma)$, but we do know some of them, by the results of [34]; in particular we know all of the $\Omega(a, b)$ with $a-b \leq 3$; see Figure 17 .

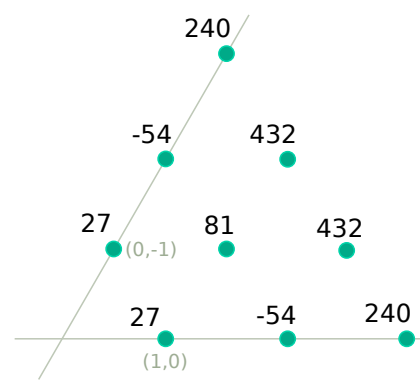

Figure 17: Some degeneracies of BPS particles in the $T_{3}$ theory with $u>0$. Each green dot represents a charge $\gamma=(a, b)=a \gamma_{A}+b \gamma_{B}$, and is plotted at the point $-Z_{\gamma} \in \mathbb{C}$, and decorated by the BPS count $\Omega(\gamma) \in \mathbb{Z}$. The charges shown are the ones with $\arg \left(-Z_{\gamma}\right) \in$ $\left[0, \frac{\pi}{3}\right]$, and with the smallest values of $\operatorname{Re}\left(-Z_{\gamma} / \hbar\right)$ when $\arg \hbar \approx \frac{\pi}{6}$.

Thus we can try approximating $\mathbf{S}_{0, \frac{\pi}{3}}$ by just the contributions from these least-suppressed $\Omega(a, b)$; this gives a sequence of approximations,

$$
\begin{aligned}
& \mathbf{S}_{0, \frac{\pi}{3}}^{(1)}=\mathcal{K}_{0,-1}^{\frac{1}{2} 27} \mathcal{K}_{1,0}^{\frac{1}{2} 27}, \\
& \mathbf{S}_{0, \frac{\pi}{3}}^{(2)}=\mathcal{K}_{0,-1}^{\frac{1}{2} 27} \mathcal{K}_{0,-2}^{-\frac{1}{2} 54} \mathcal{K}_{1,-1}^{81} \mathcal{K}_{1,0}^{\frac{1}{2} 27} \mathcal{K}_{2,0}^{-\frac{1}{2} 54}, \\
& \mathbf{S}_{0, \frac{\pi}{3}}^{(3)}=\mathcal{K}_{0,-1}^{\frac{1}{2} 27} \mathcal{K}_{0,-2}^{-\frac{1}{2} 54} \mathcal{K}_{0,-3}^{\frac{1}{2} 240} \mathcal{K}_{1,-2}^{432} \mathcal{K}_{1,-1}^{81} \mathcal{K}_{2,-1}^{432} \mathcal{K}_{1,0}^{\frac{1}{2} 27} \mathcal{K}_{2,0}^{-\frac{1}{2} 54} \mathcal{K}_{3,0}^{\frac{1}{2} 240},
\end{aligned}
$$

and so on. To write the next approximation $\mathbf{S}_{0, \frac{\pi}{3}}^{(4)}$ would require us to know the BPS count $\Omega(3,-1)$, which was not computed in [34], so for now we stop here.

We have tested these approximations numerically; for example, at $\hbar=\mathrm{e}^{\frac{\pi \mathrm{i}}{6}}$ and $u=1$, we find:

\begin{tabular}{|c|c|c|}
\hline & $A$ & $B$ \\
\hline$x$ & $(-3.81327+4.08339 i) \times 10^{-3}$ & $(-1.207491+1.440995 i) \times 10^{2}$ \\
$\mathbf{S}_{0, \frac{\pi}{3}}^{(1)} x$ & $(-3.40103+4.07226 \mathrm{i}) \times 10^{-3}$ & $(-1.220866+1.303183 \mathrm{i}) \times 10^{2}$ \\
$\mathbf{S}_{0, \frac{\pi}{3}}^{(2)} x$ & $(-3.41706+4.07711 \mathrm{i}) \times 10^{-3}$ & $(-1.221625+1.308395 \mathrm{i}) \times 10^{2}$ \\
$\mathbf{S}_{0, \frac{\pi}{3}}^{(3)} x$ & $(-3.41628+4.07696 \mathrm{i}) \times 10^{-3}$ & $(-1.221619+1.308141 \mathrm{i}) \times 10^{2}$ \\
\hline$y$ & $(-3.41630+4.07694 \mathrm{i}) \times 10^{-3}$ & $(-1.221611+1.308147 \mathrm{i}) \times 10^{2}$ \\
\hline
\end{tabular}

As expected, the $\mathbf{S}_{0, \frac{\pi}{3}}^{(k)} x$ are converging to $y$ as $k$ increases. Also as expected, the speed of convergence increases as we increase $|u|$; for example, at $\hbar=\mathrm{e}^{\frac{\pi \mathrm{i}}{6}}$ and $u=10$, we find: 


\begin{tabular}{|c|c|c|}
\hline & $A$ & $B$ \\
\hline$x$ & $(2.86472-2.57616 \mathrm{i}) \times 10^{-5}$ & $(1.929843-1.734237 \mathrm{i}) \times 10^{4}$ \\
$\mathbf{S}_{0, \frac{\pi}{3}}^{(1)} x$ & $(2.86673-2.57616 \mathrm{i}) \times 10^{-5}$ & $(1.929986-1.735579 \mathrm{i}) \times 10^{4}$ \\
$\mathbf{S}_{0, \frac{\pi}{3}}^{(2)} x$ & $(2.86673-2.57616 \mathrm{i}) \times 10^{-5}$ & $(1.9299866-1.735579 \mathrm{i}) \times 10^{4}$ \\
$\mathbf{S}_{0, \frac{\pi}{3}}^{(3)} x$ & $(2.86673-2.57616 \mathrm{i}) \times 10^{-5}$ & $(1.929986-1.735579 \mathrm{i}) \times 10^{4}$ \\
\hline$y$ & $(2.86673-2.57616 \mathrm{i}) \times 10^{-5}$ & $(1.929986-1.735579 \mathrm{i}) \times 10^{4}$ \\
\hline
\end{tabular}

We regard these results as strong evidence for the consistency of the whole story.

We could also run this program in reverse: since we can compute $x$ and $y$ numerically for any given $u$ and in particular for large $|u|$, we could try to determine the BPS counts $\Omega(\gamma)$ from the condition that $\mathbf{S}_{0, \frac{\pi}{3}} x=y$. It is easy in this way to "discover" the fact that $\Omega(1,0)=\Omega(0,-1)=27$, and in principle one could iteratively determine the higher $\Omega(a, b)$ by the same strategy. As $a-b$ increases, so does the needed precision in the numerical computations of $x$ and $y$.

With our confidence thus bolstered, we tried writing down approximate versions of the desired integral equation (2.19), taking $\vartheta(\arg \hbar)=\arg \hbar$, but truncating as follows: we fix some $k$, and then include only the $\Omega(a, b)$ shown in Figure 17 with $a-b \leq k$, together with their images under the obvious $\mathbb{Z}_{6}$ symmetry. It is not clear a priori whether the resulting approximate equations have any right to work; nevertheless, we tried solving them numerically anyway, with the following results:

\begin{tabular}{|c|c|c|c|}
\hline \multirow{6}{*}{$u=5:$} & & $A$ & $B$ \\
\hline & $\mathcal{X}^{(0)}$ & $11.59062 \times 10^{-5}$ & $(0.005041+0.92884 i) \times 10^{2}$ \\
\hline & $\mathcal{X}^{(1)}$ & $8.42628 \times 10^{-5}$ & $(0.308629+1.04475 \mathrm{i}) \times 10^{2}$ \\
\hline & $\mathcal{X}^{(2)}$ & $8.00913 \times 10^{-5}$ & $(0.362395+1.05700 \mathrm{i}) \times 10^{2}$ \\
\hline & $\mathcal{X}^{(3)}$ & $7.87397 \times 10^{-5}$ & $(0.380959+1.06060 \mathrm{i}) \times 10^{2}$ \\
\hline & $\mathcal{X}$ & $7.77949 \times 10^{-5}$ & $(0.394281+1.06300 \mathrm{i}) \times 10^{2}$ \\
\hline \multirow{6}{*}{$u=1:$} & & $A$ & $B$ \\
\hline & $\mathcal{X}^{(0)}$ & $4.99201 \times 10^{-3}$ & $(0.17298+1.40473 i) \times 10^{1}$ \\
\hline & $\mathcal{X}^{(1)}$ & $2.93480 \times 10^{-3}$ & $(1.07747+1.49881 \mathrm{i}) \times 10^{1}$ \\
\hline & $\mathcal{X}^{(2)}$ & $2.68345 \times 10^{-3}$ & $(1.23050+1.48742 \mathrm{i}) \times 10^{1}$ \\
\hline & $\mathcal{X}^{(3)}$ & $2.60470 \times 10^{-3}$ & $(1.28429+1.47980 \mathrm{i}) \times 10^{1}$ \\
\hline & $\mathcal{X}$ & $2.55054 \times 10^{-3}$ & $(1.32318+1.47307 \mathrm{i}) \times 10^{1}$ \\
\hline \multirow{6}{*}{$u=0.01:$} & & $A$ & $B$ \\
\hline & $\mathcal{X}^{(0)}$ & $31.92335 \times 10^{-2}$ & $(0.97281+1.47856 \mathrm{i}) \times 10^{0}$ \\
\hline & $\mathcal{X}^{(1)}$ & $7.03803 \times 10^{-2}$ & $(3.62200+1.043866 \mathrm{i}) \times 10^{0}$ \\
\hline & $\mathcal{X}^{(2)}$ & $5.00813 \times 10^{-2}$ & $(4.35361+1.00679 \mathrm{i}) \times 10^{0}$ \\
\hline & $\mathcal{X}^{(3)}$ & $4.38490 \times 10^{-2}$ & $(4.67643+0.96774 \mathrm{i}) \times 10^{0}$ \\
\hline & $\mathcal{X}$ & $3.91347 \times 10^{-2}$ & $(4.98408+0.84366 i) \times 10^{0}$ \\
\hline
\end{tabular}


In each of these tables, $\mathcal{X}_{\gamma}^{(k)}$ is the value computed numerically from the $k$-th truncated integral equation, and $\mathcal{X}_{\gamma}$ is the value computed numerically from the monodromy of the $T_{3}$ equation. These results offer some support for the conjecture that $\lim _{k \rightarrow \infty} \mathcal{X}_{\gamma}^{(k)}=\mathcal{X}_{\gamma}$.

Rather than studying these successive approximations, what would be really desirable would be to give a closed formula for $\mathbf{S}_{0, \frac{\pi}{3}}$; then we could write down a version of the integral equation (2.19) which would compute the exact $\mathcal{X}_{\gamma}$. This remains as a problem for the future.

\subsection{Spectral problem}

Finally, we briefly consider a spectral problem for the $T_{3}$ equation, analogous to those we considered for the Mathieu equation in Subsection 4.4 and Subsection 4.9: we search for those $u^{\prime}$ such that the $T_{3}$ equation admits a discrete $\mathcal{W}$-abelianization with

$$
\mathcal{X}_{A}=1
$$

We recall that for large $u^{\prime}$ the asymptotics of 3 of the 4 discrete $\mathcal{W}$-abelianizations are given by (6.26). Thus a natural first place to look for solutions of (6.40) at large $u^{\prime}$ is at the $u^{\prime}$ satisfying

$$
1=\mathcal{X}_{A} \approx \exp \left(-M u^{\prime \frac{1}{3}}\right),
$$

where $u^{\prime \frac{1}{3}}$ is allowed to be any of the three cube roots. This leads to potential solutions at

$$
u^{\prime} \approx 8 \pi^{3} \mathrm{i} n^{3} / M^{3}= \pm 1.666221 \mathrm{i}, \pm 13.32977 \mathrm{i}, \pm 44.9880 \mathrm{i}, \pm 106.6381 \mathrm{i}, \ldots
$$

By numerical experimentation we find actual solutions at

$$
u^{\prime} \approx \pm 0.0610186 \mathrm{i}, \pm 2.148003 \mathrm{i}, \pm 14.24769 \mathrm{i}, \pm 46.3655 \mathrm{i}, \pm 108.4752 \mathrm{i}, \ldots
$$

which asymptotically indeed appear to approach the values (6.42).

The reader might find our choice of spectral problem a little unmotivated, since its very formulation involves the spectral coordinates $\mathcal{X}_{A}$. It might be some comfort to know that the solutions of (6.40) can be alternatively described as points $u^{\prime}$ for which

$$
\operatorname{Tr} \mathbf{A} \mathbf{B}^{-1}-\operatorname{Tr} \mathbf{B} \mathbf{A}^{-1}= \pm 12 \sqrt{3} \mathbf{i},
$$

as one sees by substituting (6.40) into the monodromy matrices (6.21). In the parlance of exact WKB, one would say (6.40) is the "exact quantization condition" for the solutions of (6.44). One could also go the other way, starting with one's favorite condition on the matrices A, B, C and finding the corresponding exact quantization condition in terms of the spectral coordinates $\mathcal{X}_{A}, \mathcal{X}_{B}$; we have not explored in this direction. 


\section{Supersymmetric field theory}

In the main part of this paper we have been exploring the exact WKB method for certain differential equations (opers) of order 2 and 3 . In this final section we consider the relation of our constructions to $\mathcal{N}=2$ supersymmetric quantum field theories of class $S$ in four spacetime dimensions. Our discussion here is somewhat open-ended; we hope to return to these questions in the future.

\subsection{Opers and QFT of class $S$}

Fixing a Lie algebra $\mathfrak{g}$ and a punctured Riemann surface $C$ with singularity data at the punctures determines an $\mathcal{N}=2$ theory $\mathfrak{X}(\mathfrak{g}, C)$ of class $S$. It has been known for some time that there is a connection between the theory $\mathfrak{X}(\mathfrak{g}, C)$ and the space of $\mathfrak{g}$-opers on $C$; see e.g. $[35,81,18,82]$ for various aspects of this connection. In this section we describe a slightly different version of the connection.

The Coulomb branch of the theory $\mathfrak{X}(\mathfrak{g}, C)$ is the base $\mathcal{B}_{0}(\mathfrak{g}, C)$ of the Hitchin integrable system. The algebra $\mathcal{A}_{0}$ of chiral local operators in theory $\mathfrak{X}(\mathfrak{g}, C)$ is canonically identified with the space of holomorphic functions on $\mathcal{B}_{0}(\mathfrak{g}, C)$. Following [35], suppose we deform the theory by turning on the " $\frac{1}{2} \Omega$-background" associated to a rotation in the $x_{2}-x_{3}$ plane, with parameter $\varepsilon=\hbar$. This modification deforms $\mathcal{A}_{0}$ into a new algebra $\mathcal{A}_{\hbar}$, consisting of supersymmetric local operators inserted at the origin of the $x_{2}-x_{3}$ plane, still free to move in the $x_{0}$ and $x_{1}$ directions. $\mathcal{A}_{\hbar}$ can be thought of as the algebra of functions on a deformation $\mathcal{B}_{\hbar}(\mathfrak{g}, C)$ of $\mathcal{B}_{0}(\mathfrak{g}, C)$. By studying the Hilbert space of the theory on $S^{3}$ and using the state-operator map, together with known facts about how $S$-duality acts in the theory reduced on $S^{1}$, one can show that the deformed space $\mathcal{B}_{\hbar}(\mathfrak{g}, C)$ is canonically isomorphic to the space of $\mathfrak{g}$-opers on $C .^{32}$ So, in short, turning on the $\frac{1}{2} \Omega$-background deforms the Coulomb branch into the space of opers.

This deformation might sound a bit trivial since, when considered simply as complex manifolds, the Coulomb branch and the space of opers are isomorphic; however, the two spaces come equipped with natural presentations in terms of holomorphic functions, which are different in the two cases, as we will discuss below.

The three spaces of opers we considered in this paper correspond in this way to familiar quantum field theories:

\begin{tabular}{|c|c|c|c|}
\hline opers & $\mathfrak{g}$ & $C$ & theory $\mathfrak{X}(\mathfrak{g}, C)$ \\
\hline cubic potential (3.1) & $A_{1}$ & $\mathbb{C P}^{1}$, irregular puncture & $\left(A_{1}, A_{2}\right)$ Argyres-Douglas theory \\
Mathieu (4.1) & $A_{1}$ & $\mathbb{C P}^{1}$, 2 irregular punctures & $\mathcal{N}=2$ Yang-Mills, $G=\mathrm{SU}(2)$ \\
$T_{3}$ equation (6.1) & $A_{2}$ & $\mathbb{C P}^{1}, 3$ regular punctures & $E_{6}$ Minahan-Nemeschansky \\
\hline \hline
\end{tabular}

\footnotetext{
${ }^{32}$ We thank David Ben-Zvi for explaining this point to us.
} 


\subsection{Spectral coordinates as vevs}

The stars of this paper are the spectral coordinate functions $\mathcal{X}_{\gamma}(\hbar)$ on $\mathcal{B}_{\hbar}(\mathfrak{g}, C)$. What is their meaning in the theory $\mathfrak{X}(\mathfrak{g}, C)$ ?

The function

$$
\widetilde{Z}_{\gamma}(\hbar)=\hbar \log \mathcal{X}_{\gamma}(\hbar)
$$

is a deformation of the function $Z_{\gamma}$ on $\mathcal{B}_{0}(\mathfrak{g}, C)$ (if we momentarily ignore the multivaluedness of the $\log$ ). Since $Z_{\gamma}$ is the vev of the vector multiplet scalar $a_{\gamma}$, we suspect that $\widetilde{Z}_{\gamma}(\hbar)$ is likewise the vacuum expectation value of an operator $\widetilde{a}_{\gamma}(\hbar)$. The operator $\widetilde{a}_{\gamma}(\hbar)$ should be a deformation of $a_{\gamma}$ which preserves supersymmetry in the $\frac{1}{2} \Omega$-background. Such a deformation might not be simple to construct; nevertheless, a posteriori, the WKB expansion (2.15) of $\mathcal{X}_{\gamma}(\hbar)$ suggests that there is a universal $\widetilde{a}_{\gamma}(\hbar)$ to all orders in $\hbar$.

What about going beyond series in $\hbar$ ? We have seen that the $\mathcal{X}_{\gamma}(\hbar)$ can be defined beyond perturbation theory in various ways, corresponding to the different choices of spectral network. One particularly interesting nonperturbative definition is the function we called $\mathcal{X}_{\gamma}^{\mathrm{RH}}(\hbar)$ in $\S 2.6$, with the canonical choice (2.20). Thus we conjecture that this canonical choice corresponds to a canonical nonperturbative definition of $\widetilde{a}_{\gamma}(\hbar)$.

This canonical $\widetilde{a}_{\gamma}(\hbar)$ must have some new features compared to $a_{\gamma}$ :

- $\widetilde{a}_{\gamma}(\hbar)$ should suffer from a nonperturbative discontinuity as a function of $\hbar$ whenever there exists a BPS state whose central charge is aligned with $\hbar$, corresponding to the fact that the functions $\mathcal{X}_{\gamma}^{\mathrm{RH}}(\hbar)$ jump at the active rays. We might interpret this as saying that the operators $\widetilde{a}_{\gamma}(\hbar)$ are defined only in the IR (like the $a_{\gamma}$ ), and the scale below which this IR description is appropriate goes to zero as $\hbar$ approaches an active ray.

- $\widetilde{a}_{\gamma}(\hbar)$ should also suffer from an additive ambiguity, because $\widetilde{Z}_{\gamma}(\hbar)$ has an ambiguity by shifts by $2 \pi \mathrm{i} \hbar$. This ambiguity presumably comes from the possibility of shifting by a local operator built from background supergravity fields. (After dimensional reduction to $\mathcal{N}=(2,2)$ theory in the $x^{0}-x^{1}$ plane, the rotation in the $x^{2}-x^{3}$ plane becomes a global symmetry; then $\hbar$ can be interpreted as a complex twisted mass for this global symmetry, and the ambiguity we are after would come from shifting by the scalar in the background vector multiplet.)

It would be very interesting to give a direct construction of the operator $\widetilde{a}_{\gamma}(\hbar)$ and to understand more precisely why it has the above features.

\subsection{Scaling line defects}

Although we do not have a direct construction of the operators $\widetilde{a}_{\gamma}(\hbar)$ in hand, we can at least propose a construction which should yield the operators $\exp \left(\widetilde{a}_{\gamma}(\hbar) / \hbar\right)$, as follows.

We recall that in an $\mathcal{N}=2$ theory one has families of $\frac{1}{2}$-BPS line defects $L(\zeta)$ labeled by a parameter $\zeta \in \mathbb{C}^{\times}$. It was argued in [83] that in the low-energy limit of the theory there 
exist distinguished $\frac{1}{2}$-BPS "IR line defects" $L_{\gamma}$. The vacuum expectation values of these line defects on $\mathbb{R}^{3} \times S^{1}$ are functions $\hat{\mathcal{X}}_{\gamma}(R, \zeta)$ which are close analogues of the functions $\mathcal{X}_{\gamma}^{\mathrm{RH}}(\hbar)$; the precise relation was proposed in [26],

$$
\lim _{R \rightarrow 0} \hat{\mathcal{X}}_{\gamma}(R, \zeta=\hbar R)=\mathcal{X}_{\gamma}^{\mathrm{RH}}(\hbar) .
$$

So far this is only a relation on the level of functions; can we promote it to the level of operators?

Here is a possible approach. After the $\Omega$-background deformation in the $x_{2}-x_{3}$ plane we expect that, for any $R>0, L_{\gamma}$ can be wrapped supersymmetrically around the circle $\left(x_{2}\right)^{2}+\left(x_{3}\right)^{2}=R^{2}{ }^{33}$ Taking the limit $R \rightarrow 0$ then gives a supersymmetric local operator placed at the origin of the $x_{2}-x_{3}$ plane, which we propose to identify with $\exp \left(\widetilde{a}_{\gamma}(\hbar) / \hbar\right)$.

To get a different viewpoint on this construction, following [81], we can deform the $x_{2}-x_{3}$ plane to a "cigar" metric and then compactify on the radial circle. The result is a 3dimensional theory on a half-space, with a boundary condition corresponding to the origin of the $x_{2}-x_{3}$ plane. At low energies the 3-dimensional theory is described by a sigma model into a moduli space $\mathcal{M}(\mathfrak{g}, C, \hbar)$ of flat $\mathfrak{g}$-connections on $C$, and it was proposed in [81] that the boundary condition we get corresponds to a Lagrangian subspace $\mathcal{L}_{\text {oper }} \subset \mathcal{M}(\mathfrak{g}, C, \hbar)$, whose points are the opers. ${ }^{34}$ This is consistent with our proposal, as follows. Wrapping $L_{\gamma}$ around the compactification circle gives a local operator $O_{\gamma}$ in the sigma model. As we approach the boundary the radius of the compactification circle shrinks to zero, so at the boundary our proposal says $O_{\gamma}$ should become identified with $\exp \left(\widetilde{a}_{\gamma}(\hbar) / \hbar\right)$. This is what the $\mathcal{L}_{\text {oper }}$ boundary condition enforces: it requires that the $O_{\gamma}$ obey the same relations as the $\exp \left(\widetilde{a}_{\gamma}(\hbar) / \hbar\right)$.

\footnotetext{
${ }^{33}$ Here is a heuristic way to understand why $L_{\gamma}$ can be wrapped supersymmetrically around the circle. Suppose $\hbar$ is real. We imagine lifting the 4-dimensional theory to a 5-dimensional theory on an $\mathbb{R}^{4}$ bundle over $S^{1}$, where the $S^{1}$ base has length $\rho$, and the $x_{2}-x_{3}$ plane in the fiber is rotated by an angle $\rho \hbar$ as we go around the $S^{1}$ base. In the limit $\rho \rightarrow 0$ this gives rise to an effectively 4-dimensional theory, which can be identified with the $\Omega$-background deformation of the original theory. On the other hand, this 5-dimensional background is locally Euclidean space, and in the 5-dimensional theory, we can put the line defect $L_{\gamma}$ supersymmetrically on any straight line. We choose a straight line in the $x_{4}$ direction, beginning at some point $\left(x_{0}, x_{1}, x_{2}, x_{3}, x_{4}=0\right)$. After going around the $S^{1}$ fiber this line will return to $\left(x_{0}, x_{1}, x_{2}^{\prime}, x_{3}^{\prime}, x_{4}=0\right)$ where $\left(x_{2}^{\prime}, x_{3}^{\prime}\right)$ is the image of $\left(x_{2}, x_{3}\right)$ under rotation by an angle $\rho \hbar$. If $\rho \hbar=\frac{2 \pi}{N}$, then after going around $N$ times, the line closes up to a loop, which pierces the $\mathbb{R}^{4}$ fiber in $N$ points arranged around a circle in the $x_{2}-x_{3}$ plane. In the limit as $\rho \rightarrow 0$ ie $N \rightarrow \infty$, these $N$ points just look like a line wrapped around the circle.

${ }^{34}$ By a change of variable introduced in [81], $\mathcal{M}(\mathfrak{g}, C, \hbar)$ can be identified with the moduli space of the theory without $\Omega$-background, compactified on a circle of radius $R=|\hbar|^{-1}$. This moduli space is hyperkähler, with complex structures labeled by $\zeta \in \mathbb{C P}^{1}$; the boundary condition we get preserves the subalgebra labeled by $\zeta=\frac{\hbar}{|\hbar|}$.
} 


\subsection{Opers and instanton counting}

Concretely, what are the relations obeyed by the local operators $\widetilde{a}_{\gamma}(\hbar)$, or by their vevs $\widetilde{Z}_{\gamma}(\hbar)$ ?

The functions $Z_{\gamma}$ on $\mathcal{B}_{0}(\mathfrak{g}, C)$ obey well-known relations: choosing a symplectic basis $\left\{A_{1}, \ldots, A_{r}, B^{1}, \ldots, B^{r}\right\}$ for the charge lattice $\Gamma$, the $Z_{B}$ are determined by the $Z_{A}$, via the formula

$$
Z_{B^{I}}=\partial \mathcal{F}\left(Z_{A_{1}}, \ldots, Z_{A_{r}}\right) / \partial Z_{A_{I}}
$$

for a locally defined holomorphic function $\mathcal{F}$ called "prepotential." The existence of such an $\mathcal{F}$ reflects the fact that $Z$ gives a local Lagrangian embedding of the Coulomb branch $\mathcal{B}_{0}$ into the symplectic vector space $\Gamma^{*} \otimes \mathbb{C}$. Physically, $\mathcal{F}$ gives a Lagrangian description of the $\mathcal{N}=2$ theory on its Coulomb branch.

At $\hbar \neq 0$ there is a very similar picture: any log spectral coordinate system $\widetilde{Z}_{\gamma}$ gives local Darboux coordinates on the moduli space $\mathcal{M}(\mathfrak{g}, C, \hbar)$, and the fact that $\mathcal{L}_{\text {oper }}$ is a Lagrangian subspace means that there is a locally defined $\widetilde{\mathcal{F}}$ for which $\mathcal{L}_{\text {oper }}$ is given by the equations ${ }^{35}$

$$
\widetilde{Z}_{B^{I}}=\partial \widetilde{\mathcal{F}}\left(\widetilde{Z}_{A_{1}}, \ldots, \widetilde{Z}_{A_{r}}, \hbar\right) / \partial \widetilde{Z}_{A_{I}} .
$$

Now it is natural to ask: what is the meaning of $\widetilde{\mathcal{F}}$ in the language of supersymmetric field theory? In [25] this question was considered in the special case where $\mathfrak{g}=A_{1}$ and the $\widetilde{Z}_{\gamma}$ are complexified Fenchel-Nielsen coordinates, like those we considered in Subsection 4.3 above. In this case (as long as $C$ has only regular punctures), the theory $\mathfrak{X}(\mathfrak{g}, C)$ is a supersymmetric gauge theory [29], and so one can formulate the Nekrasov instanton partition function $\mathbf{Z}\left(\varepsilon_{1}, \varepsilon_{2} ; a\right)[84,85]$. The proposal of [25] is that $\widetilde{\mathcal{F}}$ is the $\varepsilon_{2} \rightarrow 0$ limit of $\mathbf{Z}$, or more precisely,

$$
\widetilde{\mathcal{F}}\left(\widetilde{Z}_{A}, \hbar=\varepsilon_{1}\right)=\frac{1}{\varepsilon_{1}} \lim _{\varepsilon_{2} \rightarrow 0} \varepsilon_{2} \log \mathbf{Z}\left(\varepsilon_{1}, \varepsilon_{2} ; a=\varepsilon_{1} \widetilde{Z}_{A}\right) .
$$

The formula (7.5) is a direct link between two very different-looking objects: on the LHS the monodromy of SL(2)-opers on the Riemann surface $C$, on the RHS equivariant integrals over moduli of instantons in $\mathbb{R}^{4}$. It has been extended in $[18,82]$ to a broader class of Lagrangian field theories of class $S$; in those cases the LHS involves monodromy of SL $(N)$ opers on $C$, expressed in terms of $\widetilde{Z}_{A}$ which are higher-rank analogues of complexified Fenchel-Nielsen coordinates.

It is difficult to check (7.5) directly. Nevertheless, in [25, 18] evidence for (7.5) has been given, and in [82] a proof in many cases. The strategy is as follows. In Lagrangian field theories of class $S$ one always has parameters $q_{i}$ which can be varied: from the field theory point of view these are gauge couplings, while from the point of view of $C$ they are

\footnotetext{
${ }^{35}$ In conformal theories $\widetilde{\mathcal{F}}$ depends only on the $\widetilde{Z}_{A_{i}}$ and not on $\hbar$. In non-conformal theories there are complex parameters $m_{i}$ with the dimension of mass, and then $\widetilde{\mathcal{F}}$ depends on $\hbar$ through the combinations $m_{i} / \hbar$.
} 
moduli of the complex structure. One considers a degeneration limit " $q_{i} \rightarrow 0$ ": in field theory this is a weak-coupling limit, and in the complex moduli space of $C$ it is a limit where $C$ maximally degenerates to a chain of three-punctured spheres. Expanding both sides of (7.5) in powers of the $q_{i}$, each term is a well-defined nonperturbative function of $\varepsilon_{1}$. Thus the statement (7.5) is sensitive to the precise nonperturbative definition of $\widetilde{Z}_{\gamma}$, and as is shown in $[25,18,82]$, it holds only when one takes the $\widetilde{Z}_{\gamma}$ to be complexified Fenchel-Nielsen coordinates (or their higher-rank analogues).

In Section 6 of this paper, we have been exploring a specific coordinate system $\widetilde{Z}_{\gamma}$ which arose naturally from the exact WKB analysis of the locus of opers associated to the $\frac{1}{2} \Omega$-deformed $E_{6}$ Minahan-Nemeschansky theory. One might ask whether some analogue of (7.5) holds in this setting. To formulate this question sharply would require us to understand precisely how to define $\mathbf{Z}$ in the non-Lagrangian Minahan-Nemeschansky theory. We suspect that the proper formulation of $\mathbf{Z}$ in a general non-Lagrangian field theory requires a choice of boundary condition, and that there is a natural class of boundary conditions corresponding to the different spectral coordinate systems $\widetilde{Z}_{\gamma}$; thus in a general theory the equality (7.5) could indeed hold, with both sides depending on this choice of boundary condition. We hope to develop this story more fully in the future.

\section{A Computations of spectral coordinates}

In this appendix we give some computations omitted from the main text.

\section{A.1 Computations for the cubic potential}

Computation of (3.3). We will only describe the computation for $\mathcal{X}_{A}$; that for $\mathcal{X}_{B}$ is similar.

We need to compute the parallel transport of $\nabla^{\mathrm{ab}}$ along a path in the homology class $\gamma_{A}$. To compute concretely it is convenient to work relative to bases of $\nabla^{\mathrm{ab}}$-flat sections in each domain. Each local $\nabla^{\mathrm{ab}}$-flat section corresponds to a local $\nabla$-flat section, and by continuation we can think of all these local flat sections as lying in a single 2-dimensional vector space $V$, the space of global $\nabla$-flat sections over the plane. See Figure 18. 


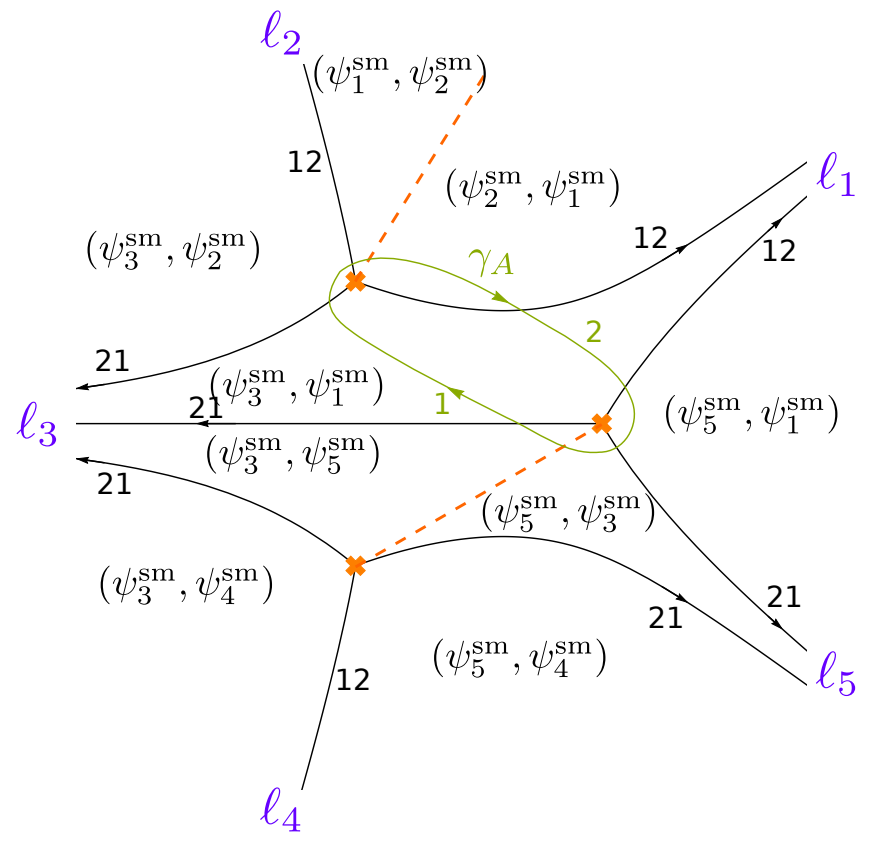

Figure 18: The Stokes graph from Figure 4, with the local WKB bases shown in each domain. To write the basis concretely as an ordered pair of solutions we have used the trivialization of the double cover $\Sigma$ away from branch cuts; thus, in a domain containing a branch cut, we write two versions of the basis, one on each side of the cut.

Relative to these local bases, the parallel transport within each domain is just represented by 1 , and the only nontrivial part is the gluing factor from (2.11):

- When we cross a single wall of type $i j$ on sheet $i$, from side $L$ to side $R$, we get a factor

$$
\frac{\left[\psi_{i}^{L}, \psi_{j}^{L}\right]}{\left[\psi_{i}^{R}, \psi_{j}^{L}\right]} .
$$

- When we cross a single wall of type $i j$ on sheet $j$, we also get a gluing factor, but this factor is just 1 if $\psi_{j}^{L}=\psi_{j}^{R}$, which it always is in this example.

The representative of $\gamma_{A}$ shown in Figure 18 crosses six walls; multiplying the factors for these six crossings, starting from the eastmost region, gives

$$
\mathcal{X}_{A}=\frac{\left[\psi_{5}^{\mathrm{sm}}, \psi_{1}^{\mathrm{sm}}\right]}{\left[\psi_{5}^{\mathrm{sm}}, \psi_{3}^{\mathrm{sm}}\right]} \times 1 \times 1 \times \frac{\left[\psi_{3}^{\mathrm{sm}}, \psi_{2}^{\mathrm{sm}}\right]}{\left[\psi_{1}^{\mathrm{sm}}, \psi_{2}^{\mathrm{sm}}\right]} \times 1 \times 1
$$

matching (3.3) as desired.

\section{A.2 Computations for the Mathieu equation}

Computation of (4.5). We need to compute the parallel transport of $\nabla^{\mathrm{ab}}$ along a path in the homology class $\gamma_{B}$. We use the path given in Figure 6. 
As above, it is convenient to work relative to bases of $\nabla^{\mathrm{ab}}$-flat sections in each domain. See Figure 19. Again by continuation we think of all these local flat sections as lying in a single 2-dimensional vector space $V$. In this case there is an added technical difficulty: the monodromy around $z=0$ means there are no global $\nabla$-flat sections. Instead we identify $V$ as the space of $\nabla$-flat sections on the complement of the blue dashed line ("monodromy cut").

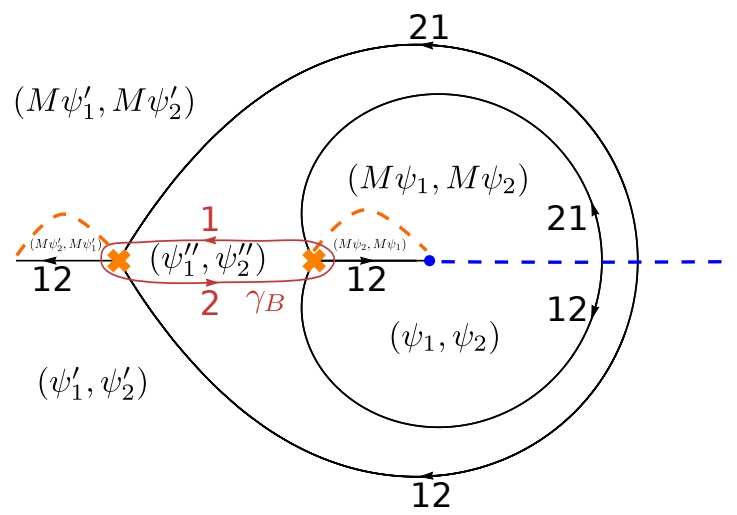

Figure 19: The Stokes graph from Figure 6, with the local WKB bases shown in each domain. As before, to write the basis concretely as an ordered pair of solutions we have used the trivialization of the double cover $\Sigma$ away from branch cuts; thus, in a domain containing a branch cut, we write two versions of the basis, one on each side of the cut. When we cross the monodromy cut, the local WKB basis of $\nabla^{\mathrm{ab}}$-flat sections does not change, but the way we identify them with elements of $V$ does jump, by the action of the monodromy $M$.

Again the only nontrivial part of the parallel transport is the gluing factors appearing in (2.11), (2.12), When we cross a double wall on sheet $i$, from side $L$ to side $R$, we get a factor

$$
\sqrt{\frac{\left[\psi_{i}^{L}, \psi_{j}^{L}\right]}{\left[\psi_{i}^{R}, \psi_{j}^{R}\right]} \frac{\left[\psi_{i}^{L}, \psi_{j}^{R}\right]}{\left[\psi_{i}^{R}, \psi_{j}^{L}\right]^{L}}},
$$

and when we cross a single wall of type $i j$ on sheet $i$, from side $L$ to side $R$, we get a factor

$$
\frac{\left[\psi_{i}^{L}, \psi_{j}^{L}\right]}{\left[\psi_{i}^{R}, \psi_{j}^{L}\right]} .
$$

We can further simplify these factors by choosing bases with $\left[\psi_{1}, \psi_{2}\right]=1,\left[\psi_{1}^{\prime}, \psi_{2}^{\prime}\right]=1$, $\left[\psi_{1}^{\prime \prime}, \psi_{2}^{\prime \prime}\right]=1$. Then starting from the southwest corner, the gluing factors we encounter are

$$
\mathcal{X}_{B}=\sqrt{\frac{\left[\psi_{2}^{\prime}, \psi_{1}^{\prime \prime}\right]}{\left[\psi_{2}^{\prime \prime}, \psi_{1}^{\prime}\right]}} \times \sqrt{\frac{\left[\psi_{2}^{\prime \prime}, \psi_{1}\right]}{\left[\psi_{2}, \psi_{1}^{\prime \prime}\right]}} \times 1 \times \sqrt{\frac{\left[M \psi_{1}, \psi_{2}^{\prime \prime}\right]}{\left[\psi_{1}^{\prime \prime}, M \psi_{2}\right]}} \times \sqrt{\frac{\left[\psi_{1}^{\prime \prime}, M \psi_{2}^{\prime}\right]}{\left[M \psi_{1}^{\prime}, \psi_{2}^{\prime \prime}\right]}} \times 1 .
$$

Using $M \psi_{1}^{\prime \prime}=\mu \psi_{1}^{\prime \prime}, M \psi_{2}^{\prime \prime}=\mu^{-1} \psi_{2}$, and $M \psi_{1}=\psi_{2}, M \psi_{1}^{\prime}=\psi_{2}^{\prime}$, this reduces to

$$
\mathcal{X}_{B}=\frac{\left[\psi_{1}, \psi_{2}^{\prime \prime}\right]}{\left[\psi_{1}, \psi_{1}^{\prime \prime}\right]} \frac{\left[\psi_{1}^{\prime}, \psi_{1}^{\prime \prime}\right]}{\left[\psi_{1}^{\prime}, \psi_{2}^{\prime \prime}\right]}
$$


which matches the desired (4.5).

Computation of (4.21). Just as above, all we need to compute are the gluing factors along the paths $\gamma_{A}$ and $\gamma_{B}$, with respect to the bases shown in Figure 20. We can choose

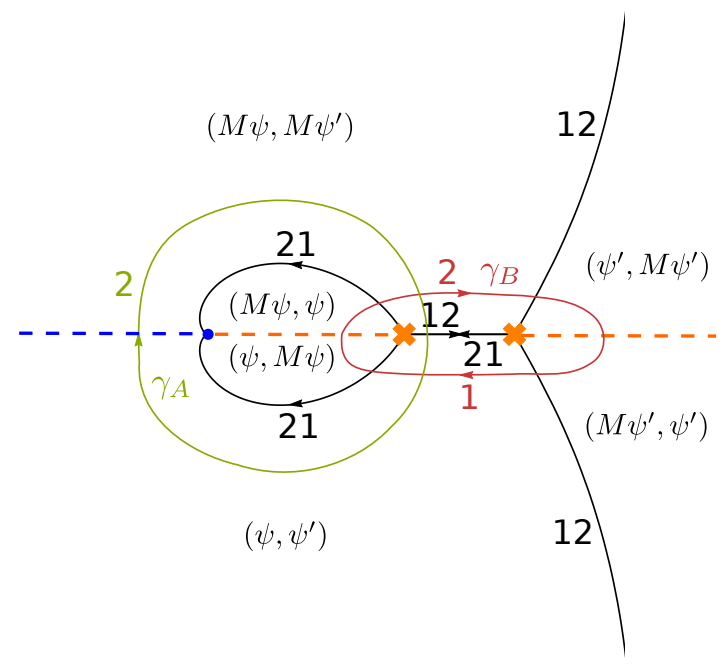

Figure 20: The Stokes graph from Figure 8, with local WKB bases shown in each domain. All notation is as in Figure 19 above.

$\left[\psi, \psi^{\prime}\right]=1$ to simplify. In going around $\gamma_{A}$ we only meet one wall, with the gluing factor

$$
\mathcal{X}_{A}= \pm \sqrt{\frac{\left[M \psi^{\prime}, \psi\right]}{\left[\psi^{\prime}, M \psi\right]}}
$$

To fix the branch we would need to carefully implement the WKB prescription from Subsection 2.3, which we do not do here.

For $\gamma_{B}$ the product of gluing factors, starting from the southeast, is

$$
\mathcal{X}_{B}=\frac{\left[M \psi^{\prime}, \psi^{\prime}\right]}{\left[\psi, \psi^{\prime}\right]} \times 1 \times \frac{[M \psi, \psi]}{\left[M \psi, M \psi^{\prime}\right]} \times 1=\frac{\left[M \psi^{\prime}, \psi^{\prime}\right][M \psi, \psi]}{\left[\psi, \psi^{\prime}\right]^{2}} .
$$

The results (A.7), (A.8) match the desired (4.21).

\section{A.3 Computations for the $T_{3}$ equation}

Abelianizations and adapted bases. Suppose we have a $\mathcal{W}$-abelianization of the $T_{3}$ equation. Then we can choose bases compatible with the $\mathcal{W}$-abelianization in the various domains of Figure 21, as shown.

In writing the form of these bases we began by labeling the basis in the middle as $\left(\psi_{1}, \psi_{2}, \psi_{3}\right)$ and then used the facts that:

- According to (5.6) the $k$-th projective basis element does not change when we cross a wall of type $i j$ and $j i$ (this implies e.g. that the first basis element in the northeast region must be $\psi_{1}$ ), 


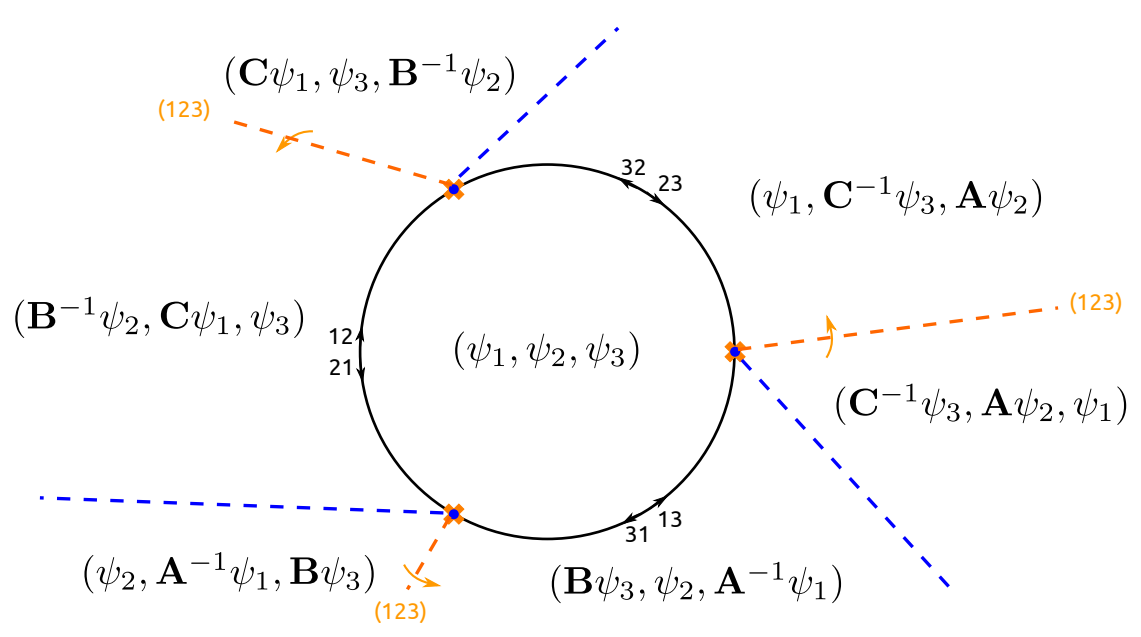

Figure 21: The Stokes graph from Figure 10, with local WKB bases shown in each domain. The notation is as in the figures above.

- Crossing a branch cut of the covering $\Sigma \rightarrow C$ (orange in Figure 21) permutes the projective basis elements,

- The projective bases on the two sides of a monodromy cut (blue in Figure 21) differ by the monodromy (A, B or C) attached to the cut.

One key fact remains to be used: again by (5.6), for a wall of type $i j$ and $j i$, the plane spanned by the $i$-th and $j$-th basis elements is the same on both sides of the wall. Applying this to the northeast wall, which is of type 23 and 32 , leads to the condition that

$$
\left\langle\psi_{2}, \psi_{3}\right\rangle=\left\langle\mathbf{C}^{-1} \psi_{3}, \mathbf{A} \psi_{2}\right\rangle
$$

which is (6.3b); doing similarly for the other two walls gives the other two parts of (6.3). Thus, the basis $\left(\psi_{1}, \psi_{2}, \psi_{3}\right)$ is indeed a basis in special position. Conversely, given a basis $\left(\psi_{1}, \psi_{2}, \psi_{3}\right)$ in special position, the local bases shown in Figure 21 give a $\mathcal{W}$-abelianization. This shows the claimed identification between $\mathcal{W}$-abelianizations and bases in special position.

Computation of (6.18). As above, all we need to compute are the gluing factors along the paths representing $\gamma_{A}$ and $\gamma_{B}$ shown in Figure 13. These factors are given by (5.6): for a wall of type $i j$ and $j i$, and a path on sheet $i$, the factor is

$$
\sqrt{\frac{\left[\psi_{i}^{L}, \psi_{j}^{L}, \psi_{k}^{L}\right]}{\left[\psi_{i}^{R}, \psi_{j}^{R}, \psi_{k}^{L}\right]} \frac{\left[\psi_{i}^{L}, \psi_{j}^{R}, \psi_{k}^{L}\right]}{\left[\psi_{i}^{R}, \psi_{j}^{L}, \psi_{k}^{L}\right]}} .
$$

Since all the walls are double, we will not need to use (5.5) anywhere.

For $\gamma_{A}$ the computation is particularly simple: only two of the four crossings give a nontrivial factor, namely the places where the path crosses the 23-32 wall. This gives 
directly

$$
\begin{aligned}
\mathcal{X}_{A} & =\sqrt{\frac{\left[\psi_{2}, \mathbf{A} \psi_{2}, \psi_{1}\right]}{\left[\mathbf{A} \psi_{2}, \mathbf{C}^{-1} \psi_{3}, \psi_{1}\right]} \frac{\left[\psi_{2}, \psi_{3}, \psi_{1}\right]}{\left[\mathbf{A} \psi_{2}, \psi_{2}, \psi_{1}\right]}} \times \sqrt{\frac{\left[\psi_{3}, \mathbf{C}^{-1} \psi_{3}, \psi_{1}\right]}{\left[\mathbf{C}^{-1} \psi_{3}, \mathbf{A} \psi_{2}, \psi_{1}\right]} \frac{\left[\psi_{3}, \psi_{2}, \psi_{1}\right]}{\left[\mathbf{C}^{-1} \psi_{3}, \psi_{3}, \psi_{1}\right]}} \\
& =\frac{\left[\psi_{2}, \psi_{3}, \psi_{1}\right]}{\left[\mathbf{C}^{-1} \psi_{3}, \mathbf{A} \psi_{2}, \psi_{1}\right]}
\end{aligned}
$$

matching (6.18a) as desired. The computation giving $\mathcal{X}_{B}$ is similar but a little longer since three of the four crossings give nontrivial factors: thus we have altogether 6 factors in numerator and denominator; one common factor cancels, leaving the desired (6.18b).

\section{References}

[1] R. Balian, G. Parisi, and A. Voros, "Quartic oscillator," in Feynman path integrals (Proc. Internat. Colloq., Marseille, 1978), vol. 106 of Lecture Notes in Phys., pp. 337-360. Springer, Berlin-New York, 1979.

[2] A. Voros, Spectre de l'équation de Schrödinger et méthode BKW, vol. 9 of Publications Mathématiques d'Orsay 81 [Mathematical Publications of Orsay 81]. Université de Paris-Sud, Département de Mathématique, Orsay, 1982.

[3] A. Voros, "The return of the quartic oscillator: the complex WKB method," Ann. Inst. H. Poincaré Sect. A (N.S.) 39 (1983), no. 3, 211-338.

[4] H. J. Silverstone, "JWKB connection-formula problem revisited via Borel summation," Phys. Rev. Lett. 55 (1985), no. 23, 2523-2526.

[5] T. Kawai and Y. Takei, Algebraic analysis of singular perturbation theory, vol. 227 of Translations of Mathematical Monographs. American Mathematical Society, Providence, RI, 2005. Translated from the 1998 Japanese original by Goro Kato, Iwanami Series in Modern Mathematics.

[6] Y. Takei, "WKB analysis and Stokes geometry of differential equations," in Analytic, algebraic and geometric aspects of differential equations, Trends Math., pp. 263-304. Birkhäuser/Springer, Cham, 2017.

[7] K. Iwaki and T. Nakanishi, "Exact WKB analysis and cluster algebras," J. Phys. A 47 (2014), no. 47, 474009, 98, 1401.7094.

[8] S. Hirose, "On the redundant and non-redundant virtual turning points for the AKT equation," in Several aspects of microlocal analysis, RIMS Kôkyûroku Bessatsu, B57, pp. 39-59. Res. Inst. Math. Sci. (RIMS), Kyoto, 2016.

[9] S. Sasaki, "A bifurcation phenomenon of Stokes curves around a double turning point, and influence of virtual turning points upon the transition probabilities for three-level systems," Adv. Math. 301 (2016) 711-738. 
[10] N. Honda, T. Kawai, and Y. Takei, Virtual turning points, vol. 4 of SpringerBriefs in Mathematical Physics. Springer, Tokyo, 2015.

[11] A. Shudo, "A role of virtual turning points and new Stokes curves in Stokes geometry of the quantum Hénon map," in Algebraic analysis of differential equations from microlocal analysis to exponential asymptotics, pp. 251-264. Springer, Tokyo, 2008.

[12] T. Aoki, N. Honda, T. Kawai, T. Koike, Y. Nishikawa, S. Sasaki, A. Shudo, and Y. Takei, "Virtual turning points-a gift of microlocal analysis to the exact WKB analysis," in Algebraic analysis of differential equations from microlocal analysis to exponential asymptotics, pp. 29-43. Springer, Tokyo, 2008.

[13] A. Shudo, "Virtual turning points and new Stokes curves in Stokes geometry of quantum Hénon map," Nonlinear Phenom. Complex Syst. 10 (2007), no. 1, 41-50.

[14] T. Aoki, T. Kawai, S. Sasaki, A. Shudo, and Y. Takei, "Virtual turning points and bifurcation of Stokes curves for higher order ordinary differential equations," J. Phys. A 38 (2005), no. 15, 3317-3336.

[15] D. Gaiotto, G. W. Moore, and A. Neitzke, "Wall-crossing, Hitchin systems, and the WKB approximation," 0907.3987.

[16] D. Gaiotto, G. W. Moore, and A. Neitzke, "Spectral networks," Annales Henri Poincaré 14 (Nov., 2013) 1643-1731, 1204.4824.

[17] L. Hollands and A. Neitzke, "Spectral networks and Fenchel-Nielsen coordinates," Letters in Mathematical Physics 106 (2016), no. 6, 1312.2979.

[18] L. Hollands and O. Kidwai, "Higher length-twist coordinates, generalized heun's opers, and twisted superpotentials," http://arxiv.org/abs/1710.04438v1.

[19] I. Coman, E. Pomoni, and J. Teschner, "From quantum curves to topological string partition functions," $1811.01978 \mathrm{v} 2$.

[20] N. Nikolaev, "Abelianisation of logarithmic $\mathfrak{s l}_{2}$-connections," $1902.03384 v 1$.

[21] V. Fock and A. Goncharov, "Moduli spaces of local systems and higher Teichmüller theory," Publ. Math. Inst. Hautes Études Sci. (2006), no. 103, 1-211, math/0311149.

[22] D. G. L. Allegretti, "Voros symbols as cluster coordinates," 1802.05479v1.

[23] S. P. Tan, "Complex Fenchel-Nielsen coordinates for quasi-Fuchsian structures," Internat. J. Math. 5 (1994), no. 2, 239-251.

[24] Y. Kabaya, "Parametrization of $\operatorname{PSL}(2, C)$-representations of surface groups," Geometriae Dedicata 170, Issue 1 (2011) 9-62, 1110.6674.

[25] N. Nekrasov, A. Rosly, and S. Shatashvili, “Darboux coordinates, Yang-Yang functional, and gauge theory," 1103.3919.

[26] D. Gaiotto, "Opers and TBA," 1403.6137. 
[27] D. Gaiotto, G. W. Moore, and A. Neitzke, "Spectral networks and snakes," Annales Henri Poincaré 15 (Jan., 2014) 61-141, 1209. 0866.

[28] J. A. Minahan and D. Nemeschansky, "An $N=2$ superconformal fixed point with $E_{6}$ global symmetry," Nucl. Phys. B482 (1996) 142-152, hep-th/9608047.

[29] D. Gaiotto, "N = 2 dualities," 0904.2715.

[30] P. Dorey and R. Tateo, "Anharmonic oscillators, the thermodynamic Bethe ansatz, and nonlinear integral equations," J.Phys.A32:L419-L425,1999 (Dec., 1998) hep-th/9812211v1.

[31] P. Dorey, C. Dunning, and R. Tateo, "The ODE/IM correspondence," J.Phys.A40:R205,2007 (Mar., 2007) http://arxiv.org/abs/hep-th/0703066v2.

[32] T. Bridgeland, "Riemann-Hilbert problems from Donaldson-Thomas theory," $1611.03697 \mathrm{v} 2$.

[33] K. Ito, M. Mariño, and H. Shu, “TBA equations and resurgent Quantum Mechanics," 1811.04812.

[34] L. Hollands and A. Neitzke, "BPS states in the Minahan-Nemeschansky $E_{6}$ theory," Comm. Math. Phys. 353 (2017), no. 1, 317-351, 1607.01743.

[35] N. A. Nekrasov and S. L. Shatashvili, "Quantization of Integrable Systems and Four Dimensional Gauge Theories," 0908.4052.

[36] L. F. Alday, D. Gaiotto, and Y. Tachikawa, "Liouville correlation functions from four-dimensional gauge theories," Lett. Math. Phys. 91 (2010) 167-197, 0906.3219.

[37] A. Mironov and A. Morozov, "Nekrasov functions and exact Bohr-Sommerfeld integrals," JHEP 1004:040,2010 (Oct., 2009) 0910.5670v2.

[38] A. Mironov and A. Morozov, "Nekrasov functions from exact BS periods: the case of SU(N)," J.Phys.A43:195401,2010 (Nov., 2009) 0911.2396v1.

[39] D. Krefl, “Non-perturbative quantum geometry," http://arxiv.org/abs/1311.0584v1.

[40] A.-K. Kashani-Poor and J. Troost, "Pure $N=2$ super yang-mills and exact WKB," $1504.08324 \mathrm{v} 1$.

[41] S. K. Ashok, D. P. Jatkar, R. R. John, M. Raman, and J. Troost, "Exact WKB analysis of $N=2$ gauge theories," 1604.05520v1.

[42] S. Codesido and M. Marino, "Holomorphic anomaly and quantum mechanics," http://arxiv. org/abs/1612.07687v3.

[43] S. Codesido, M. Marino, and R. Schiappa, "Non-perturbative quantum mechanics from non-perturbative strings," 1712.02603v2. 
[44] O. Dumitrescu, L. Fredrickson, G. Kydonakis, R. Mazzeo, M. Mulase, and A. Neitzke, "From the Hitchin section to opers through nonabelian Hodge," 1607.02172. To appear in J. Diff. Geom.

[45] G. Álvarez, C. J. Howls, and H. J. Silverstone, "Dispersive hyperasymptotics and the anharmonic oscillator," Journal of Physics A: Mathematical and General 35 (apr, 2002) 4017-4042.

[46] G. Álvarez and C. Casares, "Exponentially small corrections in the asymptotic expansion of the eigenvalues of the cubic anharmonic oscillator," Journal of Physics A: Mathematical and General 33 (jul, 2000) 5171-5182.

[47] G. Álvarez, "Langer-Cherry derivation of the multi-instanton expansion for the symmetric double well," Journal of Mathematical Physics 45 (2004), no. 8, 3095-3108.

[48] G. V. Dunne and M. Unsal, "Generating non-perturbative physics from perturbation theory," Phys. Rev. D 89, 041701(R) (2014) (June, 2013) 1306.4405v2.

[49] G. V. Dunne and M. Unsal, “Uniform WKB, multi-instantons, and resurgent trans-series," Phys. Rev. D 89, 105009 (2014) (Jan., 2014) 1401.5202v2.

[50] G. Basar, G. V. Dunne, and M. Unsal, "Quantum geometry of resurgent perturbative/nonperturbative relations," 1701.06572v1.

[51] S. Jeong, "Splitting of surface defect partition functions and integrable systems," Nucl.Phys.B 938, (Sept., 2017) 775-806, 1709.04926.

[52] D. Gaiotto, G. W. Moore, and A. Neitzke, "Wall-crossing in coupled 2d-4d systems," JHEP 12 (2012) 1103. 2598.

[53] W. M. Goldman, "Convex real projective structures on compact surfaces," J. Differential Geom. 31 (1990), no. 3, 791-845.

[54] I. Coman, E. Pomoni, and J. Teschner, "Toda conformal blocks, quantum groups, and flat connections," $1712.10225 \mathrm{v} 2$.

[55] A. Neitzke and N. Nikolaev. To appear.

[56] J. Écalle, Introduction aux fonctions analysables et preuve constructive de la conjecture de Dulac. Actualités Mathématiques. [Current Mathematical Topics]. Hermann, Paris, 1992.

[57] J. Écalle, "Six lectures on transseries, analysable functions and the constructive proof of Dulac's conjecture," in Bifurcations and periodic orbits of vector fields (Montreal, PQ, 1992), vol. 408 of NATO Adv. Sci. Inst. Ser. C Math. Phys. Sci., pp. 75-184. Kluwer Acad. Publ., Dordrecht, 1993.

[58] E. Delabaere and F. Pham, "Resurgent methods in semi-classical asymptotics," Ann. Inst. H. Poincaré Phys. Théor. 71 (1999), no. 1, 1-94.

[59] D. Dumas and A. Neitzke. To appear. 
[60] A. Goncharov and L. Shen, "Donaldson-Thomas transformations of moduli spaces of G-local systems," 1602.06479.

[61] M. A. Evgrafov and M. V. Fedorjuk, "Asymptotic behavior of solutions of the equation $w^{\prime \prime}(z)-p(z, \lambda) w(z)=0$ as $\lambda \rightarrow \infty$ in the complex $z$-plane," Uspehi Mat. Nauk 21 (1966), no. 1 (127), 3-50.

[62] Y. Sibuya, Global theory of a second order linear ordinary differential equation with a polynomial coefficient. North-Holland Publishing Co., Amsterdam-Oxford; American Elsevier Publishing Co., Inc., New York, 1975. North-Holland Mathematics Studies, Vol. 18.

[63] E. Delabaere and F. Pham, "Unfolding the quartic oscillator," Ann. Physics 261 (1997), no. $2,180-218$.

[64] É. Delabaere, H. Dillinger, and F. Pham, "Résurgence de Voros et périodes des courbes hyperelliptiques," Ann. Inst. Fourier (Grenoble) 43 (1993), no. 1, 163-199.

[65] P. Dorey, C. Dunning, and R. Tateo, "Spectral equivalences, Bethe Ansatz equations, and reality properties in PT-symmetric quantum mechanics," J.Phys.A34:5679-5704,2001 (Mar., 2001) hep-th/0103051v5.

[66] A. D. Shapere and C. Vafa, "BPS structure of Argyres-Douglas superconformal theories," hep-th/9910182.

[67] A. B. Zamolodchikov, "Thermodynamic Bethe ansatz in relativistic models. Scaling three state Potts and Lee-Yang models," Nucl. Phys. B342 (1990) 695-720.

[68] G. V. Dunne and M. Unsal, "WKB and resurgence in the Mathieu equation," pages 249-298, in "Resurgence, Physics and Numbers", F. Fauvet et al (Eds), Edizioni Della Normale (2017) (Mar., 2016) 1603.04924v1.

[69] W. He and Y.-G. Miao, "Magnetic expansion of Nekrasov theory: the SU(2) pure gauge theory," Phys.Rev.D82:025020,2010 (June, 2010) 1006.1214v3.

[70] J. N. L. Connor, T. Uzer, and R. A. Marcus, “Eigenvalues of the Schrödinger equation for a periodic potential with nonperiodic boundary conditions: A uniform semiclassical analysis," J. Chem. Phys. 80 (1984) 5095.

[71] J. Zinn-Justin and U. D. Jentschura, "Multi-instantons and exact results I: Conjectures, WKB expansions, and instanton interactions," Annals Phys. 313 (2004) 197-267, quant-ph/0501136.

[72] J. Zinn-Justin and U. D. Jentschura, "Multi-instantons and exact results II: Specific cases, higher-order effects, and numerical calculations," Annals Phys. 313 (2004) 269-325, quant-ph/0501137.

[73] D. Gaiotto, G. W. Moore, and A. Neitzke, "Four-dimensional wall-crossing via three-dimensional field theory," Commun. Math. Phys. 299 (2010) 163-224, 0807. 4723. 
[74] H. L. Berk, W. M. Nevins, and K. V. Roberts, "New Stokes' line in WKB theory," Journal of Mathematical Physics 23 (1982), no. 6, 988-1002.

[75] T. Aoki, T. Kawai, and Y. Takei, "New turning points in the exact WKB analysis for higher-order ordinary differential equations,".

[76] T. Aoki, T. Kawai, T. Koike, and Y. Takei, "On the exact WKB analysis of operators admitting infinitely many phases," Adv. Math. 181 (2004), no. 1, 165-189.

[77] A. Fenyes, "A dynamical perspective on shear-bend coordinates," 1510.05757v2.

[78] I. Dolgachev, "Lectures on Cremona transformations." Available at http://www. math.lsa.umich.edu/ idolga/cremonalect.pdf.

[79] B. Keller, "Cluster algebras and derived categories," 1202.4161.

[80] M. Kontsevich and Y. Soibelman, "Stability structures, motivic Donaldson-Thomas invariants and cluster transformations," 0811.2435.

[81] N. Nekrasov and E. Witten, “The Omega deformation, branes, integrability, and Liouville theory," 1002.0888.

[82] S. Jeong and N. Nekrasov, "Opers, surface defects, and Yang-Yang functional," 1806.08270.

[83] D. Gaiotto, G. W. Moore, and A. Neitzke, "Framed BPS states," Adv. Theor. Math. Phys. 17 (2013), no. 2, 241-397, 1006. 0146.

[84] N. A. Nekrasov, "Seiberg-Witten prepotential from instanton counting," Adv. Theor. Math. Phys. 7 (2004) 831-864, hep-th/0206161.

[85] N. A. Nekrasov and A. Okounkov, "Seiberg-Witten theory and random partitions," hep-th/0306238. 